
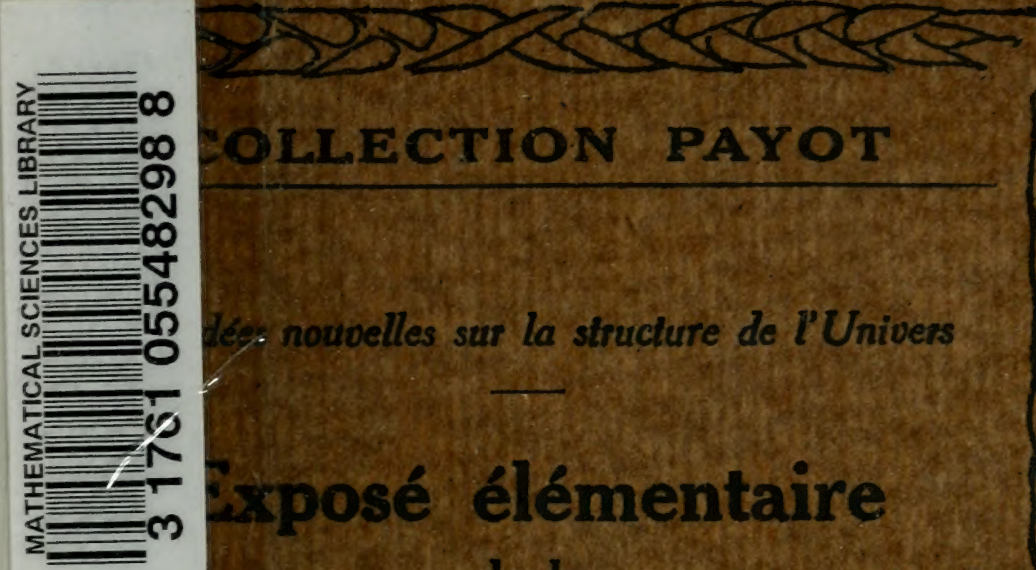

OLLECTION PAYOT

lé: nouvelles sur la structure de l'Univers

Exposé élémentaire de la

PAR

JEAN BECQUEREL

'ROFESSEUR AU MUSEUM D'HISTOIRE NATURELLE

Abec 17 figures

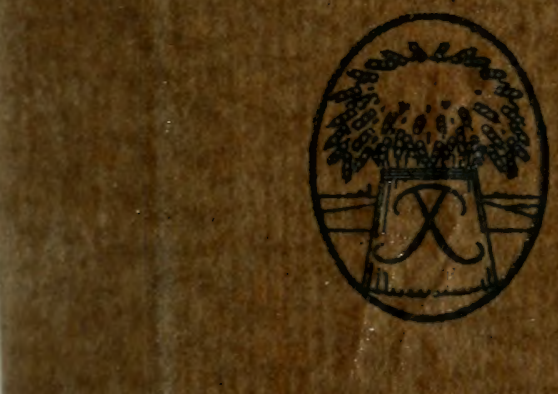

stein






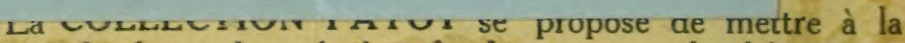
portée de chacun les principes fondamentaux et les faits essentiels dans toutes les branches du savoir humain. Elle permettra par ses exposés accessibles, clairs et précis, aux personnes instruites que les nécessités de la vie ont obligées à se spécialiser, d'être au courant des plus récentes acquisitions de la science et de l'érudition moderne.

Les ouvrages de la COLLECTION PAYOT sont conçus de manière à fournir, dans toutes les matières, à la fois une initiation pour les jeunes gens, une lecture d'un passionnant intérêt pour le grand public cultivé et un précis pour les spécialistes eux-mêmes.

№ 1. Ejounaro Montex, Professeur de langues orientales à l'Université de Genève, ancien Recteur. L'Isiam.

No 2. Camille Mauclair. Les États de la Peinture Française de 1850 à 1920.

Noi 3-4. Renś Canat, Prof, de rhétorique sup ${ }^{\text {Te }}$ au Lycée Louis-le-Grand. La Littérature Française au XIX ${ }^{e}$ siècle. Tome I (18,0-1852) Tome II (1852-1900').

$N^{0}$ 5. Louis Lecer, Membre de IInstitut, Profess ${ }^{r}$ au Collège de France. Les Anciennes Civilisations Slaves.

No 6. Paul Apperl. Membre de l'Institut, Recteur de I'Université de Paris. Éléments de la Théorie des Vecteurs et de la Géométrie analytique.

№ 7. C DE Civrisúx. La Grande Guerre (1914-1918). Aperçu d'Histoire m.litaire. 
№ 8. Henri Cordier, Membre de l'Institut. La Chine.

No 9 Ernest Babelon, Membre de l'Institut, Conserv ${ }^{r}$ du cabinet des Médailles, Professeur au Collège de France. Les Monnaies Grecques. Aperçu historiçue.

No 10. Georges Matisse, Docteur ès-sciences. Le Mouvement ScientifiQue Contemporain en France. - 1. Les Sciences Naturelles.

No 11. D Pierre Boulan, Chef du Service de radiologie et d'électrothérapie à l'hôpital de Saint-Germain. Les Agents Physiques et Ia Physiothérapie.

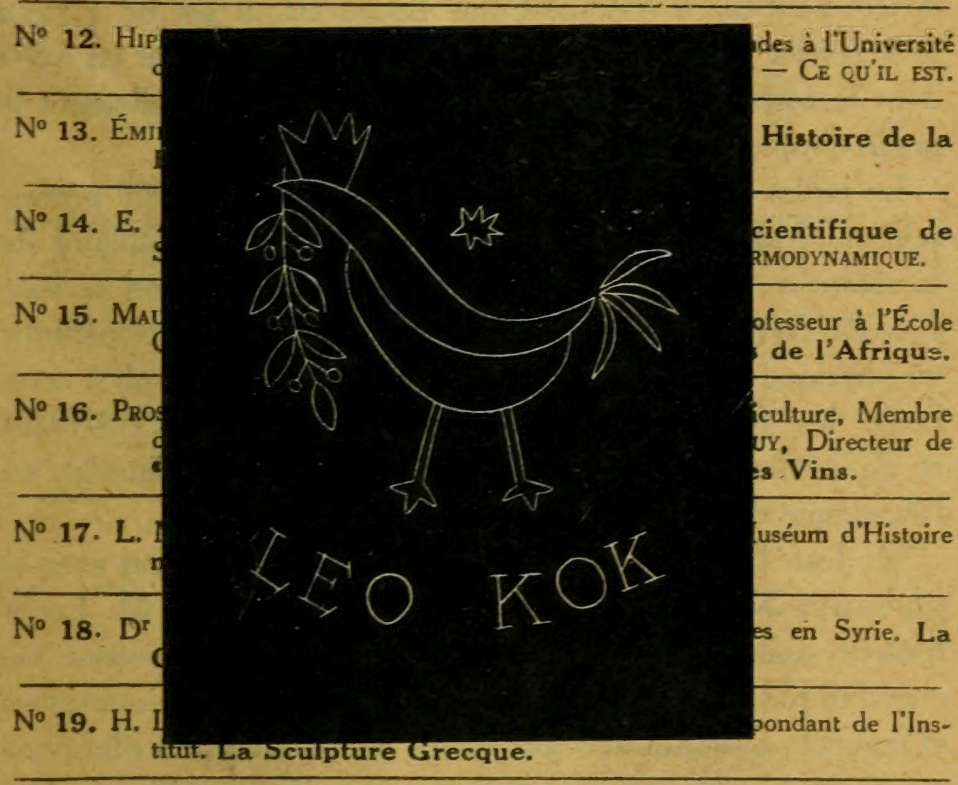

No 20. H. Andoyer, Membre de l'Académie des Sciences et du bureau des longitudes, Professeur à la Sorbonne. L'Euvre Scientifique de Laplace.

No 21. Jean Becquerel, Professeur au Musćum National d'Histoire Naturelle. Exposé élémentaire de la Théorie d'Einstein et de sa généralisation, suivi d'un Appendice à l'usage des mathématiciens.

Nos 23-24. Maurice Croiset, Membre de l'Institut, Administrateur du Collège de France. La Civilisatioń Hellénique. ApERçu hISTORI Ue.

$\mathrm{N}^{03}$ 25-26. Étienne Gilson, Chargé de Cours à la Sorbonne, Directeur d'Etu 'es à l'Ecole pratique des Hautes Etudes Religieuses. La Philosophie au Moyen Age. 


\section{JEAN BECQUEREL}

M. Jean BECQuerel appartient à une famille de physiciens : fils d'Henri Becquerel, l'illustre auteur de la découverte de la radioactivité, petit-fils d'Edmond Becquerel et de Jamin, arrière-petit-fils d'Antoine-César Becquerel, il est né à Paris le 5 février 1878. Il sortit de l'École Polytechnique en 1899 dans le corps des Ponts et Chaussées, où il a actuellement le grade d'ingénieur en chef.

En juillet 1903 il fut nommé assistant, puis en 1909 professeur au Muséum National d'Histoire Naturelle, titulaire de la chaire de Physique dans laquelle quatre Becquerel se sont succédé de père en fils depuis sa fondation (1838). Répétiteur de physique à l'École Polytechnique (1911), il a rempli à cette École les fonctions d'examinateur temporaire (1919-1920) et de professeur temporaire (1920-1921). Il est lauréat de l'Académie des Sciences (prix Rivot, prix Hughes).

M. Jean BeCQuerel, soit seul, soit en collaboration avec le professeur Kamerlingh-Onnes (de Leyde) ou avec M. Louis Matout, assistant au Muséum, a effectué de nombreuses recherches sur l'absorption de la lumière, les phénomènes optiques et magnéto-optiques aux très basses températures, la phosphorescence, les phénomènes galvano-magnétiques. Il a publié ses travaux dans les comptes rendus de l'Ac. des Sc., les C. R. de l'Ac. des Sc. d'Amsterdam (en collaboration avec le Prof. Kamerlingh-Onnes), le bulletin du Muséum, les Ann. de Ch. et de Phys., le Radium, etc...

Depuis quelques années, il s'est principalement consacré à l'étude de la théorie d'Einstein; jugeant nécessaire de défendre et de propager les idées nouvelles, il expose cette théorie à son cours du Muséum. 


\section{COLLECTION PAYOT}

\section{JEAN BECQUEREL}

PROFESSEUR AU MUSÉUM D'HISTOIRE NATURELLE

LES IDÉES NOUVELLES SUR LA STRUCTURE DE L'UNIVERS

\section{EXPOSÉ ÉLÉMENTAIRE DE LA}

\section{THÉORIE D'EINSTEIN ET DE SA GÉNÉRALISATION} SUIVI D'UN APPENDICE

A L'USAGE DEs MATHÉMATICIENS

Avec 17 figures dans le texte.



PAYOT \& C $\mathrm{CE}$, PARIS

106, BOULEVARD SAINT-GERMAIN

1922

Tous droits réservés. 


\section{TABLE DES MATIĖRES}

INTRODUCTION.

PREMIÈRE PARTIE

\section{LE PRINCIPE DE RELATIVITÉ RESTREINT}

CHAPITRE PREMIER. - LES NOTIONS ANCIENNES D'ESPACE ET DE TEMPS.

Systèmes de coordonnées, p. 11. - Le groupe de transformations de la cinématique classique (groupe de Galilée), p. 16. - Les invariants fondamentaux (temps absolu, espace absolu), p. 18. - Les bases de la dynamique newtonienne, p. 22. - Le principe de relativité de la mécanique newtonienne, p. 24.

CHAPITRE II. - LA RECHERCHE DU MOUVEMENT ABSOLU. L'EXPÉRIENCE DE MICHELSON. LE PRINCIPE DE RELATIVITÉ..

Lexpérience de Michelson, p. 27. - La contraction de Fitzgerald-Lorentz, p. 31. - Le point de vue d'Einstein, p. 33.

CHAPITRE III. - L'INVARIANCE DE LA VITESSE DE LA LUMIÈRE.

Le temps et la simultanéité, p. 34. - La vitesse de la lumière est une constante universelle, p. 36 .

Tous droits de traduction, de reproduction et d'adaptation réservés pour tous pays Copyright 1921 by Payot \& $C^{i *}$ 
CHAPITRE IV. - LA TRANSFORMATION DE LORENTZ. RELATIVITÉ DE L'ESPACE ET DU TEMPS.

Le groupe de Lorentz, p. 38. - Les lois de la mécanique doivent être compatibles avec celles de l'électromagnétisme, p. 40. - L'espace et le temps relatifs, p. 43. - La loi de composition des vitesses, p. 44. - L'expérience de Fizeau (entraînement des ondes), p. 45.

CHAPITRE V. - L'UNIVERS DE MINKOWSKI.

Union de l'espace et du temps, l'Univers quadridimensionnel, p. 48. - Propriétés des couples d'événements, p. 50. - La contraction des longueurs, p. 52. - La dilatation du temps, p. 53. - Les lignes d'univers, p. 53. - Le temps propre, p. 55. - La loi d'inertie, p. 59.

CHAPITRE VI. - DYNAMIQUE DE LA RELATIVITÉ..

La masse fonction de la vitesse, p. 60. - L'énergie et ses diverses formes, p. 62. - L'inertie de l'énergie, p. 64. - Conséquences de l'inertie de l'énergie, p. 66. - La matière réservoir d'énergie, p. 68. - Unification des principes de la dynamique : conservation de l'impulsion d'Univers, p. 69.

CHAPITRE VII. - VÉRIFICATIONS EXPÉRIMENTALES.

Les vitesses des électrons, p. 74. - Loi d'accroissement de la masse avec la vitesse, p. 74. -La structure des raies spectrales, p. 75. - Signification de l'expérience de Michelson, p. 75.

\section{DEUXIÈME PARTIE}

LE PRINCIPE DE RELATIVITÉ GÉNÉRALISÉ ET LA GRAVITATION

CHAPITRE VIII. - LE CHAMP DE GRAVITATION ET L'UNIVERS RÉEL..

Les systèmes galiléens, p. 77. - La pesanteur de l'énergie, p. 79. - L'équivalence entre un champ de gravitation et un champ de force d'inertie 
(la gravitation est une action de proche en proche ; égalité de la masse pesante et de la masse inerte; le boulet de Jules Verne; le principe d'équivalence), p. 80. - L'univers n'est pas euclidien, p. 85. - Le principe de relativité généralisé, $\mathrm{p}, 86$.

CHAPITRE IX. - LES COORDONNÉES DE GAUSS..

Le temps et les longueurs dans un champ de gravitation, p. 89. - Les surfaces et les coordonnées de Gauss, p. 91. - Extension de la théorie de Gauss, p. 96. - Courbure de l'Univers réel, p. 100.

CHAPITRE $X$. - LA LOI DE LA GRAVITATION (EINS. TEIN). .

Nature de la gravitation, p. 101. - Les tenseurs, p. 103. - La forme tensorielle des lois est exigée par le principe de relativité, p. 103. - La loi de la gravitation dans le vide, p. 105. - La loi de gravitation dans la matière, p. 108. - La loi de Newton est une approximation, p. 110.

CHAPITRE XI. - APPLICATIONS ET VÉRIFICATIONS DE LA LOI D'EINSTEIN.

Le champ de gravitation d'un centre matériel, p. 112. - Le mouvement des planètes (le déplacement du périhélie de Mercure), p. 113. - La déviation d'un rayon lumineux par le soleil, p. 113. Le déplacement des raies spectrales, p. 117.

CHAPITRE XII. - LA COURBURE DE L'ESPACE ET DU TEMPS. HYPOTHĖSES COSMOLOGIQUES..

L'espace fini bien qu'illimité, p. 118. - L'Univers d'Einstein, p. 121. - L'Univers de de Sitter, p. 123. - L'accélération et la rotation, p. 124. La structure d'Univers et l'éther, p. 125.

CONCLUSIONS GÉNÉRALES, p. 127. APPENDICE A L'USAGE DES MATHÉMATICIENS I. - RELATIVITÉ RESTREINTE.

Note 1. - Sur l'invariance de la distance spatiale de deux événements simultanés 
Note 2. - Sur les équations de la dynamique classique.

Note 3. - Sur l'expérience de Michelson et la contraction de Fitzgérald-Lorentz.

Note 4. - Remarque sur la mesure du temps.

Note 5. - Établissement des furmules du groupe de Lorentz.

Note 6. - La loi de composition des vitesses..

Note 7. - La contraction des longueurs et la dilatation du temps.

Invariance de l'hypervolume quadridimensionnel, p. 140.

Note 8. - Sur le temps propre..

Note 9. - La loi d'inertie.

Note 10. - I. Le champ électromagnétique. . . . . . . 142

Invariance de la charge électrique, p. 144. - Formules de l'effet Doppler, de l'aberration de la lumière et de la pression de radiation, p. 144.

II. Dynamique de la relativité.

La masse fonction de la vitesse (loi de Lorentz-Einstein), p. 146. - L’inertie de l'énergie (énergie cinétique, énergie rayonnante, perte de masse d'un corps qui rayonne, énergie potentielle de l'électron), p. 148. - L'impulsion d'Univers et sa conservation, p. 151.

\section{RELATIVITÉ GÉNÉRALISÉE}

Note 11. - Les tenseurs. .

Quadrivecteurs, p. 153. - Tenseurs, p. 154. - Multiplication des tenseurs, p. 155. - Contraction, p. 155. Caractère tensoriel, p. 156. - Tenseurs fondamentaux, p. 156. - Tenseurs associés, p. 157. - Longueur généralisée, condition d'orthogonalité, p. 157. - Densité tensorielle, p. 158. - Symboles de Christoffel, p. 159. - Dérivée covariante, p. 159. - Formules utiles, p. 160. - Divergence, p. 161. - Le tenseur de Riemann-Christoffel, p. 162.

Note 12. - Gravitation et dynamique..

Loi de la gravitation dans le vide, p. 163. - Théorème fondamental, p. 164. - Equations des géodésiques, p. 164. - Le tenseur impulsion-énergie ou tenseur matériel, p. 166. - La conservation de l'impulsionénergie, p. 167. - La loi de la gravitation dans la 
matière, p. 167. - Les équations de l'hydrodynamique, p. 169. - Les forces, p. 170. - La loi d'Einstein contient toute la dynamique, p. 171. - La loi de Newton, p. 172. - Propagation de la gravitation, p. 172.

Note 13. - Le champ de gravitation d'un centre matériel.

Expression de $d s^{2}$, p. 173. - Interprétation physique et réponse aux objections de M. Painlevé, p. 174. Mouvement des planètes, p. 176. - Propagation de la lumière, p. 177. - Ralentissement du temps et déplacement des raies spectrales, p. 177.

Note 14. - Les lois générales de l'électromagnétisme.

Généralisation des équations de Maxwell-Lorentz, p. 178. - La conservation de l'électricité, p. 183. - Le tenseur d'énergie électromagnétique et la loi générale de conservation, p. 183.

Note 15. - La courbure de l'espace et du temps.

La courbure non nulle dans le vide, p. 184. - L'espace fermé, p. 186. - L'Univers cylindrique d'Einstein, p. 187. - L'Univers hyperbolique de de Sitter; la barrière du temps, p. 189.

Note 16. - Généralisations de Weyl et d'Eddington.

Théorie de Weyl, p. 190. - Théorie d'Eddington, p. 195. Théorie géométrique de l'Univers, p. 196. - Identification des grandeurs physiques et des grandeurs géométriques, p. 198. - L'électron, p. 203. - Conclusions, p. 204. 


\section{INTRODUCTION}

Presque toui le monde a entendu parler de la révolution qui, depuis quelques années, a bouleversé les notions fondamentales sur lesquelles reposaient la mécanique et la physique. Je me suis efforcé, dans ce petit livre, d'exposer les grands traits de la nouvelle théorie avec le minimum de calculs, presque sans calculs, en admettant seulement que le lecteur possède les notions les plus élémentaires de géométrie et d'algèbre. J'ai fait suivre cet exposé d'un appendice où les personnes familiarisées avec le calcul différentiel trouveront une sorte de précis de la théorie mathématique.

En 1905, un jeune physicien de génic, Albert Einstein, pour expliquer l'échec de toutes les tentatives destinées à mettre en évidence le mouvement absolu de la terre dans l'espace, a cu l'audace d'abandonner les idées basées sur les apparences les plus familières. Il a développé sa théorie en deux grandes étapes : la relativité resireinte au mouvement en ligne droite avec viliesse constante, et depuis 1912 la relativité généralisée. S'étant élevé audessus de Copernic, de Galilée, et de Newton, Einstein a découvert la véritable loi de la gravitation, qui contient en elle les principes généraux de la mécanique, et a été conduit à une impressionnante conception de l'univers. 
On s'imagine à priori que "l'espace " dans lequel on observe la matic̀re et dans lequel on mesure des distances est quelque chose d'absolu. On croit aussi que "le temps est universel et absolu, que la simultanéité de deux événements a un sens bien défini; on ne voit aucun lien entre l'espace et le temps, qui apparaissent comme deux individualités bien séparées.

Ces notions doivent être abandonnées aujourd'hui. L'espace et le temps ne sont ni absolus ni indépendants: ils sont unis et forment un Univers à quatre dimensions, qui seul possède une individualité; en termes plus précis : chaque observateur décompose l'Univers en "espace " et en "temps", et deux observateurs en mouvement l'un par rappori à l'autre font deux décompositions différentes.

Chacun connait, au moins un peu, la géométrie d'Euclide; nous verrons qu'en toute rigueur l'espace-temps n'est pas régi par les lois de cette géométric, telles quion peut les étendre à une multiplicité à quatre dimensions. Une sphère, un ellipsoüde, eic., constituent des surfaces courbes auxquelles la géométrie cuclidienne du plan ne s'applique pas : de même l'Univers possède une courbure. Cette courbure se manifeste à nos yeux par le phénomène de la gravitation universelle; clle se traduit à nous par l'existence d'une force d'inertic qui nous a donné l'illusion d'une force attractive émanant de toute matière ct agissant à distance sur toute matière.

Il n'y a plus, comme dans l'ancicnne mécanique, de masse invariable caractérisant une quantité déterminée de matière. La masse se confond avec l'énergie; elle varie avec la vitesse et elle est relative à l'obseriateur car il n'y a pas de vitesse absolue, toutes les itesses de translation étant relatives. 
Enfin l'L'nivers ne doit pas être infini dans toutes ses dimensions, et la quantité totale de matière existante doit être limitée.

La mécanique classique garde son imporlance parce qu'elle constitue une approximation plus que suffisante dans la pratique, et en général satisfaisante en astronomie et en physique. Mais il est nécessaire de savoir que les notions d'espace et de temps sur lesquelles elle a été fondée sont inexactes, et d'expliquer certains écarts constatés entre les faits expérimentaux et les prévisions déduites des anciennes lois.

On doit répandre les idées nouvelles. Loin de conduire à une complication de la science, elles révèlent une admirable harmonie, une merieilleuse synthèse des lois naturelles par laquelle on aperçoit pour la première fois les liens qui unissent des phénomènes qu'on pouvait croire indépendants.

La principale difficulté qu'on rencontre dans le développement de la théorie de la relativité vient de la répugnance à abandonner des idées acquises, et de l'étonnement où l'on se trouve plongé devant certaines conséquences qui, par leur étrangeté, choquent ce que l'on considérait comme le bons sens. Je demande au lecteur d'avoir le courage, en abordaní cette étude, de renoncer résolument à toute idée préconçue.

Pour la rédaction de cet opuscule, j'ai eu recours aux mémoires de $M$. Einstein, aux conférences de $M$. P. Langevin qui a introduit en France les idées nouvelles et a beaucoup contribué à leur développement, enfin aux ouvrages de $M$. Eddington. 



\title{
PREMIÈE PARTIE
}

\section{LE PRINCIPE DE RELATIVITÉ RESTREINT}

\author{
CHAPITRE PREMIER
}

\section{LES NOTIONS A.VCIENNES D'ESP.ACE ET DE TEMPS}

L'analyse des conceptions anciennes d'espace et de temps, sur lesquelles sont fondées la géométrie et la mécanique rationnelle, nous conduira à l'expérience célèbre par laquelle Michelson avait pensé mettre en évidence le mouvement absolu de la terre dans l'espace.

J'admettrai que le lecteur possède les bases de la géométrie d'Euclide (lignes droites et lignes courbes, droites parallèles, droites perpendiculaires l'une sur l'autre, angles, etc...) ; ces bases sont d'ailleurs presque intuitives. Je rappellerai seulement ce qu'on entend par " systeme de coordonnées", en priant les lecteurs qui seraient peu familiarisés avec les mathématiques de ne pas s'effrayer de l'aridité du début de ce chapitre. Il suffit d'un peu de rénexion pour reconnaitre qu'il s'agit de notions très simples. et ces notions sont indispensables pour la comprćhension de la théorie d'Einstein.

SYSTĖMES DE CODRDONNÉEs. - ¿upposons qu une figure géométrique soit dessinée sur une feuille de papier plane. Si nous voulons préciser la forme de cette figure, il 
nous faut un moyen de repérer la position de chacun de



FiG. 1.

ses points ; d'une façon générale, l'étude des figures qu'on peut tracer sur un plan exige quion ait un moyen de désigner sans ambiguïté un point quelconque de ce plan : on y parvient à l'aide d'un système de coordonnées.

Nous pouvons, par exemple, marquer un point $O$ sur la feuille de papier et tracer, dans une direction d'ailleure arbitrairement choisie, une droite $\mathrm{O} x$ passant par ce point (fig. 1). Joignons au point $\mathrm{O}$ le point $\mathrm{A}$ que nous voulons repérer, puis mesurons la distance $\mathrm{OA}$ et l'angle que for ment les droites OA et $\mathrm{Ox}$; ces deux grandeurs, ditance $\mathrm{OA}$ et angle $\widehat{x \mathrm{OA}}$ sont dites " cocrdonnées du point A"; elles déterminent entièrement la position de point car, le "pôle" $\mathrm{O}$ et l'axe $\mathrm{O} x$ ayant été choisis une: fois pour toutes, à chaque groupe de deux coordonnées correspond un point du plan et un scul. Ces coordonnées sont appelées coordonnées polaires.

Un autre système de coordonnées, dont nous ferons constamment usage, est celui des coordonnées cartésienno: rectangulaircs. Par un point fixe $\mathrm{O}$, appelé origine des coordonnées, traçons deux droites rectangulaires $\mathrm{O} x, \mathrm{O}_{i}$ qui seront les axes de coordonnées (fig. 2). Nous pouvons. repérer tout point du plan par sa position relativement is ces axes : en effet, du point $A_{1}$ abaissons, sur ces deux axes, deux perpendiculaires; nous déteminons ainsi la projection $\mathrm{B}_{1}$ du point considéré sur l'axe $\mathrm{O} x$, el su pro- 
LES NOTIONS ANCIENNES D'ESPACE ET DE TEMPS 13 jection $\mathrm{C}_{1}$ sur l'axe $\mathrm{O}_{y}$; les distances $x_{1}=\mathrm{OB}_{1}$ et $y_{1}=O C_{1}$, comptées sur chacun des axes à partir de l'origine $\mathrm{O}$ (positivement dans un sens, négativement en sens opposé), sont les coordonnées cartésiennes du point $A$ ! Prenons maintenant un second point $A_{2 .}$, de coordonnées

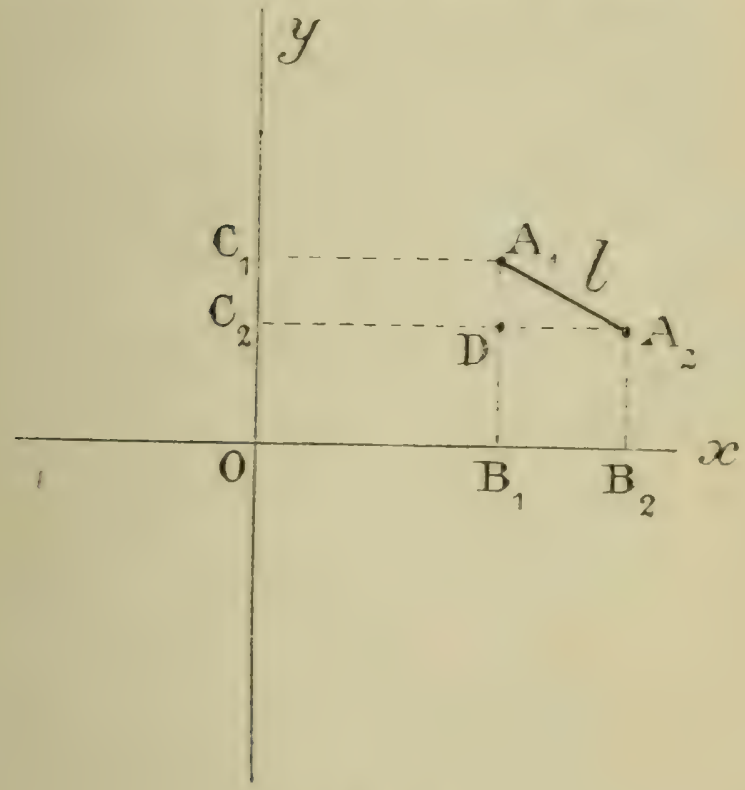

FIG. 2.

$x:$ et $y:$, et proposons-nous d'exprimer la distance des deux points $A_{1}$ et $A_{2}$ en fonction de leurs coordonnées : d'après le théorème de Pythagore (le carré construit sur l'hypoténuse d'un triangle rectangle est égal à la somme des carrés construits sur les deux autres côtés), on a

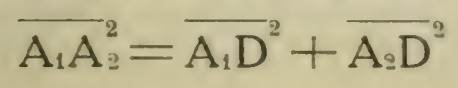


ou, en désignant par $l$ la distance $A_{1} A_{2}$ des deux points,
(1)
$L^{2}=\left(x_{2}-x_{1}\right)^{2}+\left(y_{2}-y_{1}\right)^{2}$.

Dans ce système de coordonnées, le carré de la distance de deux points est égal à la somme des carrés des différences de leurs coordonnées.

La géométrie des figures tracées sur notre feuille de papier plane est à deux dimensions, puisque deux coordonnées (deux quantités variables d'un point à un autre) sont nécessaires et suffisantes pour déterminer la position d'un point du plan. Un plan est une "multiplicité bidimensionnelle ${ }^{\prime 1}$.

Passons maintenant à la géométrie des figures tracées. non plus seulement sur un plan, mais dans l'espace; il nous faut introduire une troisième dimension : à la longueur et à la largeur vient se

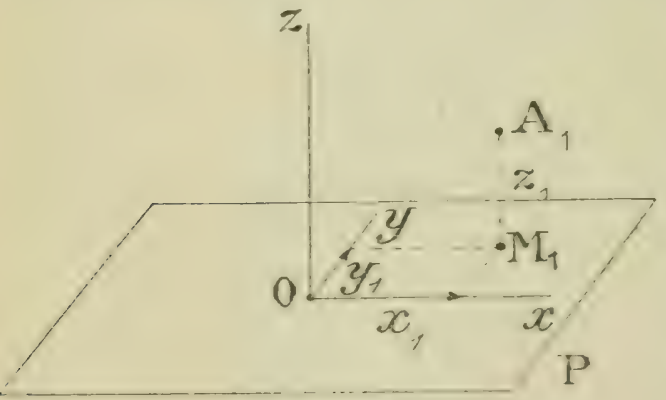

Fic. 3. joindre la hauteur. Prenons dans l'espace un plande référence $\mathrm{P}$ (représenté en perspective sur la figure 3). Dans ce plan nous pouvons, comme précédemment, choisir un point origine $\mathrm{O}$ et deux axes de coordonnées $\mathrm{O} x, \mathrm{O} y$. Soit $A_{1}$ un point quelconque de lespace; de ce point abaissons une perpen-

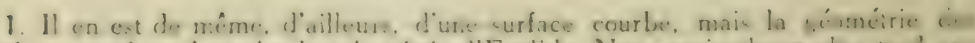


cette question. 
diculaire $A_{1} M_{i}$ sur le plan $P$; le point $A_{1}$ est entièrement défini par les coordonnées $x_{1}$ et $y_{1}$ de sa projection $\mathrm{M}_{1}$ sur le plan, auxquelles il faut joindre sa distance $z_{1}=A_{1} M_{1}$ au plan (considérée comme positive d'un côté du p'an et comme négative du côté opposé). Le point $A_{1}$ a donc trois coordonnées $x_{1}, y_{1}, z_{1}$ (coordonnées cartésiennes rectangulaires); en d'autres termes, l'espace est une "multiplicité tridimensionnelle".

La construction que nous venons de faire revient à la suivante : par un point $\mathrm{O}$ de l'espace, choisi comme origine des coordonnées, nous faisons passer trois plans rectangulaires $x \mathrm{O} y, x \mathrm{O} z, y \mathrm{O} z$ qui se coupent suivant les droites rectangulaires $\mathrm{O} x, \mathrm{O} y, \mathrm{O} z$. Les distances $x_{1}, y_{1}, z_{1}$, d'un point $\mathrm{A}_{1}$ de l'espace aux trois plans $y \mathrm{O} z, x \mathrm{O} z, x \mathrm{O}_{y}$, choisis une fois pour toutes, sont les coordonnées cartésiennes de ce point.

Par une extension facile de la formule (1), le carré de la distance de deux points $A_{1}$ et $A_{2}$ de l'espace a pour expression, en fonction des coordonnées $x_{1}, y_{1}, z_{1}$ et $x_{2}, y_{1}$, $z_{2}$ de ces deux points

$$
\text { (2) } l^{2}=\left(x_{2}-x_{1}\right)^{2}+\left(y_{2}-y_{1}\right)^{2}+\left(z_{2}-z_{1}\right)^{2} \cdot j
$$

Un premier système de coordonnées Oxyz ayant été choisi et tous les points de l'espace ayant été d'abord rapportés à ce système, nous pouvons changer de système en adoptant ensuite un second systeme $\mathrm{O}^{\prime} x^{\prime} y^{\prime} z^{\prime}$. Supposons que ce second système soit immobile par rapport au premier. Six quantités sont nécessaires et suffisantes pour définir la position relative des deux systemes d'axes : ce sont trois longueurs (les coordonnées de l'origine $\mathrm{O}^{\prime}$ du second système prises dans le premier système) qui déterminent la position relative des deux origines $\mathrm{O}$ et $\mathrm{O}^{\prime}$, et trois angles 
qui définissent l'orientation relative des axes des deux systèmes. En géomśtrie analytique, on établit les formules qui permettent, connaissant ces six quantités, de passer d'un des systèmes à l'autre, c'est-à-dire d'exprimer les coordonnées nouvelles $x, y^{\prime}, z^{\prime}$ d'un point en fonction des coordonnées anciennes $x, y, z$ du même point (et inversement).

Tous les systèmes de coordonnées (en nombre infini) immobiles les uns par rapport aux autres, constituent, à vrai dire, un seul et même système de référence (terme à retenir pour la suite), car on peut les supposer tous liés à un même corps de référence rigide. Par exemple, pour les phénomènes terrestres, il est naturel de prendre la terre comme corps de reférence et d'adopter un système quelconque de coordonnées lié à la terre ${ }^{1}$.

Le groupe de transforiations de Galilée. - Supposons maintenant que, connaissant les coordonnées d'un point dans un premier système de coordonnées $\mathrm{S}$ $(\mathrm{O} x y z)$, on demande les coordonnées du même point de l'espace dans un second systeme $\mathrm{S}^{\prime}\left(\mathrm{O}^{\prime} x^{\prime} y z^{\prime}\right)$ en mouvement par rapport au premier système.

Ici sintroduit une notion nouvelle : mouvement signifie changement de position, et ce changement implique la notion de " temps".

Considérons un système $\mathrm{S}^{\prime}\left(\mathrm{O}^{\prime} x^{\prime} y^{\prime} z^{\prime}\right)$ en mouvement rectiligne et uniforme par rapport au système $\mathrm{S}(\mathrm{O} x y z)$ c'est-à-dire se mouvant comme un ensemble rigide, par rapport à $\mathrm{S}$, en ligne droite et avec une vitesse constante $v$.

1. Il est vrai que la terre, dont l'écore présente des marées. riest pas un curps rigide, mais précisément on évalue les oscillations de l'écorce terrestre en le. rapportant à un corips de reférence fictif supposé rigicle, le géolde. 
LES NOTIONS ANCIENNES D'ESPACE ET DE TEMPS 17

Pour n'envisager que le cas le plus simple, nous supposerons que les axes des $x$ et des $x^{\prime}$ sont confondus et parallèles à la direction de la vitesse (fig. 4), que les axes des $y^{\prime}$ et des $y, \operatorname{des} z^{\prime}$ et des $z$ sont parallèles, et qu'on compte le temps $t$ à partir du moment où les deux origines $\mathrm{O}$ et $\mathrm{O}^{\prime}$ sont en coïncidence. Les formules de transformation sont évidentes: pendant le temps $t$, le point $O^{\prime}$ s'est déplacé, à partir du point $\mathrm{O}$, de la longueur $v t$ (par définition même de la vitesse qui est

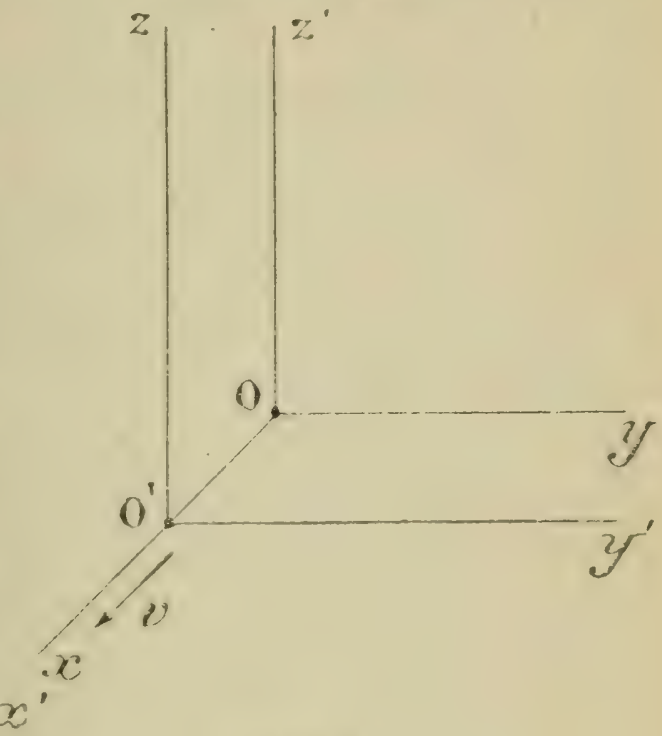

Fic. 4.

égale au quotient du trajet parcouru par le temps employé à le parcourir); donc, à l'époque $t$, la coordonnée $x^{\prime}$ d'un point, quel quil soit, est inférieure à la coordonnée $x$ du même point dans le système $\mathrm{S}$, de la longueur parcourue par $\mathrm{O}$ c'est-à-dire de $v t$; d'autre part les $y$ et les $z$ 'restent constamment égaux aux $y$ et aux $z$; on a donc (avec cette disposition particulière des axes des deux systèmes $\mathrm{S}$ et $\mathrm{S}^{\prime}$ )
(3)
$x^{\prime}=x-v t ;$
$y^{\prime}=y$;
$z^{\prime}=z$.

Ce sont les formules de transformation qui permettent de passer du système $\mathrm{S}$ au système $\mathrm{S}^{\prime}$. Ces trois relations défi- 
nissent une transformation dépendant d'un seul paramètre, la vitesse $c$, et toutes les transformations de ce genre correspondant à toutes les valeurs de $t$ constituent un groupc. c'est-à-dire que deux transformations successives de vitesses $v$ et $v^{\prime}$ équivalent à une transformation unique de même forme; on a d'ailleurs pour cette transformation unique une vitesse $v^{i \prime}=v+v$.

Ce groupe porte le nom de groupe de Galilée. Il constitue la base de la cinématique classique.

LES INVARIANTS FONDAMENTALX DAXS L'ANCIENNF. CONCEPTION DE L'LNIVERS. - La géométrie est la science des formes dans l'espace; elle ne s'occupe pas du temps: cependant, la notion d'espace et la notion de temps interviennent à la fois dans toutes nos observations, car celles-ci sont déterminées, non pas uniquement par des positions ou des formes dans l'espace, mais par le fait qu'il se passe quelque chose en un certain lieu à une certaine époque : nos observations sont donc déterminées par des cícicments. Tout événement possède quatre coordonnées : trcis coordonnées d'espace qui fixent le lieu où il s'est produit (par rapport à un corps de référence) et une crordonnée de temps (la durée écoulée à partir d'un événement origine jusquà l'époque où s'est produit l'événement considéré).

Les constatations d'événements nous conduisent à des relations entre diverses grandeurs mesurées par les observateurs; ces relations sont les lois de la physique. Il est évident que ces lois ne peurent avoir une réalité objective que si elles sont indépendantes de l'obserrateur, que si elles peuvent s'exprimer sous une forme indépendante de tout système de reférence. Notre premier souci doit itre de rechercher quels sont les ćléments incariunts, c'est-à-dire 
I.ES NOTIONS ANCIENNES DESPACE. ET DE. TEMPS 19

les grandeurs indépendantes de tout systeme de coordonnées; nous allons montrer que, dans l'ancienne conception de l'univers, il existait deux invariants fondamentaux : l'intervalle de temps écoulé entre deux événements, et la forme des figures géométriques.

LF TEMPS ABSOLU. - Dans les idées anciennes on admet que le temps est un invariant : c'est l'hypothèse Iu temps universel et absolu. Il est intéressant de chercher quelle doit être l'origine de ce postulat.

Imaginons un certain nombre de systèmes de référence en mouvement les uns par rapport aux autres; dans chaque système se trouve un observateur, immobile par rapport à son système.

Deux événements $A$ et $B$ se produisent : pour l'observateur d'un des systèmes, $A$ est antérieur à $B$. Pourquoi s'est-on cru obligé d'admettre que $\mathrm{A}$ est nécessairement antérieur à B pour tous les autres observateurs, c'est-à-dire qu'on ne peut. dans aucun cas, par un changement convenable du système de référence, inverser l'ordre de succes. sion de deux événements?

Cela tient à ce qu'on suppose implicitement que, puisque $A$ s'est montré antérieur à $B$ pour un des observateurs, il a pu être la cause de $B$, ou tout au moins qu'il aurait pu influencer $\mathrm{B}$. Comme il serait absurde de supposer que, pour d'autres observateurs, l'effet puisse être antérieur à sa cause, on est conduit à penser que l'ordre de succession de deux événements est toujours bien déterminé, quil est le méme dans tous les systemes.

Demandons-nous maintenant pourquoi on admet que $B$ a toujours pu être prévenu de $A$ : c'est parce quon suppose la possibilité d'une influence pouvant se propager instantanément. Or cette possibilité, non seulement est com- 
patible avec la mécanique ancienne, mais est exigée par cette mécanique ou lon admet la conception du solide parfait : arec une tige rigide, on aurait pu signaler instantanément la production du premier événement au point où le second ra se produire, et influencer ce second événement.

La notion de possibilité d'une propagation instantancé entraine celle de simultanéité absolue : deux événements simultanés dans un système de référence sont simultanés dans tous les autres. Il résulte de là que, pour des événements non simultanés, la durée qui sépare deux événements $A$ et $B$ est la méme pour tous les observateurs en mouvement les uns par rapport aux autres : considérons, en effet. deux systemes $\mathrm{S}$ et $\mathrm{S}^{\prime}$ dans chacun desquels les observateurs ont des korloges identiques. Prenons A commé événement origine du temps dans chacun des systemes: B se produit à l'époque $t$ du système $S$ et à l'époque $I$ du système $S$ : la simultancité étant absolue, les indications $t$ et $t$ des deux horloges constituent deux événements simulanés, non-si:ulement pour les ohserrateurs des systemes $S$ et $S^{\prime}$. mais pour tout olservateur; cela signifie que les heures marquées sont les mêmes pour tous les observateurs : lintervalle de temps séparant $\mathrm{A}$ et $\mathrm{B}$ est absolu.

On woit que les notions de solide parfait (corps rigide) de propagation instantanée, de simultanéité alisolue. de durce alssolue sunissent et sadaptent iomplètement les unes aux autres. Qu une de ces notions vienne à ètre renrerece, tout lechafaudage seerroulera.

L'ESPACI ABSOLU. - La notion despace absolu de. rive nussi de lidée du solide parfait, ou encore de linvariance de forme des figures géométriques.

Nous avons dit plus haut que la gémétrie ne siocrupe 
LES NOTIONS ANCIENNES D'ESPACE ET DE. TEMPS 21

pas du temps; en termes plus précis, nous pouvons dire qu'elle envisage seulement des événements simultanés, car la forme d'un objet est l'ensemble des positions simultanées de tous ses points (définition de M. P. Langevin). Si l'on admet que la simultanéité est absolue, une figure géométrique a une forme absolue, indépendante de l'état de mouvement du système de référence : par exemple un corps qui a la forme d'une sphère pour un observateur doit, d'après les idées anciennes, être encore une sphère pour tout observateur en mouvement par rapport au premier.

Nous trouvons alors un invariant fondamental de l'espace dans la distance spatiale de deux événements, à condition toutefois que ces événements soient simultanés (appendice, note 1).

D'autres invariants sont d'ailleurs envisagés en géométrie : ce sont les angles, surfaces, volumes.

Les équations qui expriment les lois de la géométrie sont sous la forme requise pour que ces lois soient objectives, car elles ne changent pas de forme par application des formules de transformation de coordonnées quand on passe d'un système de coordonnées à un autre.

Cette invariance de forme correspond à une réalité indépendante de tout système de référence : l'espace de la géométrie euclidienne, l'espace absolu.

Lorsque deux événements ne sont pas simultanés, leur distance spatiale cesse d'être un invariant : elle dépend du système de référence. Par exemple: un observateur quitte un lieu $\mathrm{A}$ dans un véhicule qui le transporte dans un lieu $\mathrm{B}$. Le départ de $\mathrm{A}$ et l'arrivée en $\mathrm{B}$ sont deux événements; quelle est leur distance dans l'espace? cela dépend du système de référence: dans un système lié à la terre, cette distance est la distance des deux points de la terre $\mathrm{A}$ et B; 
dans un système de coordonnées lié à l'observateur, l. distance est nulle, puisque les points de ce système nit st: sont passés les érénements sont en coïncidence.

Ainsi, la distance de deux événements non simultanés cst relative au système de référence. Sans doute, sil y a un espace absolu, il doit y avoir une distance absolue dans cet espace, mais l'cheservateur ne peut pas la connaitre parce que, ignorant son propre mouvement dans l'esprace alssolu, il ne peut pas tenir compte du trajet quili a parcouru pendant le temps écoulé entre les deux événements.

Voilà un résuitat étrange et peu satisfaisant pour l'csprit : la cinématique classique fait envisager une dissymétrie entre les propriétés de l'espace et celles du temps: l'espace que nous percevons serait absolu pour les événements simultanés, relatif pour des événements non simultanés, alors que le temps serait toujours absolu.

Cette dissymétrie disparaitra dans l'espace-temps de la théorie nouvelle.

LES BASES DE LA DINAMIQUE NEWTONIENNE - La dynamique introduit deux notions nouvelles, celle de force et celle de masse.

Tout d'abord, il existe, dans l'ancienne mécanique, un loi fondamentale appelće loi dinertie de Galilée : twut corps sur lequel niest appliquée aucune force reste au repos nu se meut danis l'espace d' un mouvement rectiligne et uniforme.

Autrement dit : la matière conserve d'elle-méme !e mourement acquis, tant qư aucune influence, appelée force, ne loblige à modifier son mourement. L'incrie est ectle tendance de la matière à garder son état de mouvement. 
Lnisqu'une force est appliquée sur un corps, le mouvement de celui-ci devient accéléré. On appelle accélération l'accroissement (positif ou négatif) de la vitesse dans l'unité de temps. Si la force agit constamment dans la direction de la vitesse acquise, la trajectoire reste rectiligne, la direction de la vitesse n'est pas modifiée mais sa grandeur est changée (accélération tangentielle); si, à chaque instant, la force agit dans une direction perpendiculaire à la trajectoire (normalement à la trajectoire), la vitesse reste constante en grandeur mais sa direction est constamment modifiéc par la force (accélération normale); si la force est oblique sur la trajectoire, il y a à la fois changement de grandeur et changement de direction de la vitesse.

Ainsi, une force produit une accélération dans la direction ou elle agit; le rapport entre la grandeur de la force agissante et la grandeur de l'accélération prise par un corps sous l'action de cette force est, par définition, la masse de ce corps ${ }^{1}$.

Dans la dynamique newtonienne, la masse d'une portion de matière est, à priori, considérée comme rigoureusement constante, indépendante des changements d'état que la portion de matière peut subir, indépendante de la vitesse : c'est un invariant qui a même valeur dans tous les sys-

1. Il faut bien se garder de confondre la masse et le poids. Le poids d'un corps e.t la force qui asit sur lui dans le champ de gravitation de la terre; il faut divisc: le noids par l'accélération due à la pesanteur pour obtenir la masse.

En un méme lieu, tous les objets tombent avec la néme vitesse (dans le vide. sinon la résistance de l'air les ralentirait inégalement); cela signifie que l'accélération due à la pesanteur est la même pour tous les corps ; à Paris, elle est ésale à 981 en unités C. G. S., c'est-à-dire que pendant chąque seconde. la vitesse déjà acquise par un corps qui tombe s'accroit d'une vitesse supplémentaire ézale à 981 centimétres par seconde. L'accédération étant la même pour tous les corps. il y a cn un míme lieu proportionnalité exacte entre les masses et les poids.

Mais comme la terre nest pas rigoureusement sphérique, le poids d'un corps n'est pas le même partout; il est un peu plus grand aux póles qu'à l'ćquateur : cependant la masse reste constante. Sur la lune, les poids des objets seraient beaucoup plus petits que sur la terre, mais les masses seraient les nêmes. 
tèmes de référence et qui caractérise une quantité déterminée de matière.

La force est une grandeur dirigée, un vectcur; l'accélération est aussi un vecteur qui, d'après ce qui précede, est dirigé suivant la direction de la force; la masse est une grandeur qui n'a pas de direction, un scalaire.

On démontre que les équations fondamentales de la dynamique conserveni leur forme quand on passe d'un système de référence à un autre système cn mouvement rectiligne et uniforme par rapport au premier (appendice, note 2). On peut les résumer par la relation vectorielle, indépendante de tout système de coordonnées

$$
\overrightarrow{m ?}=\vec{F}
$$

$\vec{F}$ et $\ddot{i}$ étant les vecteurs force et accélération, et $m$ désignant la masse de la portion de matière considéréc.

L’invariance des lois de la mécanique permet d'en donncr des énoncés intrinséques, de même que les invariants de la géométrie (distances, angles, etc.) permettent d'énoncer les théorèmes sans faire intervenir des axes de coordonnées.

LE I'RINCIPE DE RELATIVITÉ DE IA MÉCANIQUE: NEWTONIENNE. - Puisque les lois de la mécanique sont les mêmes dans tous les systèmes en mouvement de translation uniforme, il est impossible, par des expériences nécaniques faites à l'intéricur d'un systeme clos, de mellere en évidence un mouvement de translation uniforme de ec système.

Le mouvement de translation uniforme n'a donc pas un caractere ahsolu: on ne peut parler de trans!ation unilorme que relativement à un corps de référence consideré, p.ur convention, comme au repos. 
LES NOTIONS ANCIENNES D'ESPACE ET DE TEMPS 25

Ce principe de relativité est conforme à l'expérience.

Par contre, il est essentiel de remarquer que toute accélération a un caractère absolu et peut être mise en évidence par des expériences intérieures à un système; l'état d'accélération d'un système se manifeste, à l'intérieur de ce systeme, par l'existence d'une force qui, en tout point du systeme, agit sur une masse matérielle proportionnellement à cette masse ; on dit alors que dans un systeme accéléré, il règne un champ de force d'inertie. Voici un exemple bien connu : la rotation de la terre autour de la ligne des pôles est un mouvement accéléré (tout mouvement qui n'est pas à la fois rectiligne et uniforme est accéléré ; ici le mouvement est uniforme, puisque la vitesse de rotation de la terre est constante, mais il n'est pas rectiligne). Il règne alors, en tout point de la terre, un champ de forces centrifuges: la verticale n'a pas rigoureusement la même direction que si la terre était immobile; tout projectile est soumis à une force (appelée force centrifuge ccmposée) qui dévie sa trajectcire; la méme force dévie les vents (vents alizés, vents contre-alizés) dans la circulation générale de l'atmosphère ; c'est elle enfin qui a été mise en évidence par l'expérience célèbre du pendule de Foucault. Si la terre avait été perpétuellement couverte d'un manteau de nuages, empêchant de constater sa rotation par l'observation du mouvement apparent des étoiles, on aurait cependant mesuré cette rotation avec le pendule de Foucault. 
L.I RECHERCHE DU MOUVEMENT . IBSOLU. L.EXPERIE VCE DF, MCHELSON. - LE PRINCIPL DE RELATIVITÉ.

Si, par des expéricnces mécaniques à l'intérieur d'un systeme (los, il est impossible de révéler un mouvement de translation uniforme de ce systeme, il en est autremeni lorsque le systeme n'est plus clos, lorsque l'observateur peut se mettre en relation arec un milieu extérieur. Il devient alors possible de mettre en évidence et de mesurer la vitesse par rapport au milieu extérieur.

Précisément, pour expliquer la propagation de la lumiere, les physiciens avaient supposé l'existence d'un milieu doué de propriétés quasi-matérielles, l'éther, remplissant tout l'espace et pénétrant la matière. On devait donc espérer, par des expériences électromagnétiques 'ou optiques, révéler un mouvement de translation par rapport à l'éther. L'éther suidentifiant en quelque sorte avec l'espace, on a appelé ce mouvement le moutement absolu.

Imaginons que d'un point A dans l'éther parte un signal lumineux instantané. Une seconde plus tard, l'ébranlement formera une surface d'onde sphérique ayant pour centre le point A et pour rayon 300000 kilometres, puisque la vitesse de la lumière est 300000 kilometres par seconde. Suppro-

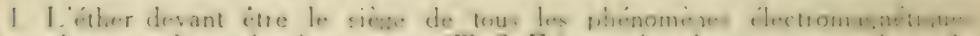

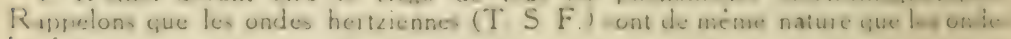
lumineuses. 
sons qu'un observateur soit parti de $A$ cn méme temps que le signal, dans la direction $\mathrm{AB}$ et aver la vitesse $v$ (fig. 5); au bout d'une seconde, il sera à la distance $\mathrm{AB}=v$; il ne se trouvera donc plus au centre de la sphère et, pour lui, la lumière ne se propagera pas avec la même vitesse dans toutes les directions: la vitesse de la lumière, relativement à cet observateur, devra être, si l'on désigne par c la vitesse



FIC. 5 . de la lumière dans l'éther (le rayon de la sphère), $\mathrm{BD}=\mathrm{c}-\boldsymbol{v}$ dans la direction de la vitesse $v, B E=c+v$ dans la direction opposée et $\mathrm{BC}=1 \mathrm{c}^{2}-v^{2}$ dans la direction perpendiculaire. L'observateur devra pouvoir constater et mesurer cet effet.

Expérience de Michelson. - Voici le principe de l'expérience que Michelson a réalisée : Un faisceau de rayons lumineux issus d'une source lumineuse et rendus sensiblement parallèles par une lentille tombe sous l'incidence de $45^{\prime \prime}$ sur une lame de verre $A$ dont la première face est légèrement argentée; cette lame réfléchit une partic du faisceau et laisse passer l'autre partie. Après réflexion normale sur les miroirs $M_{1}$ et $M_{2}$ qui sont placés sur deux bras rectangulaires, on obtient deux faisceaux qui ont parcouru, l'un le chemin $\mathrm{SOM}_{1} \mathrm{OL}$ (fig. 6), l'autre le chemin SOM.OL et qui viennent se superposer suivant la direction OL; ils sont reçus dans une lunette $L$. Tout se passe 
comme si le rayon $\mathrm{SOM}_{4} \mathrm{OL}$ avait parcouru le chemin SOM'OL, M' étant l'image, appelée plan de référence,

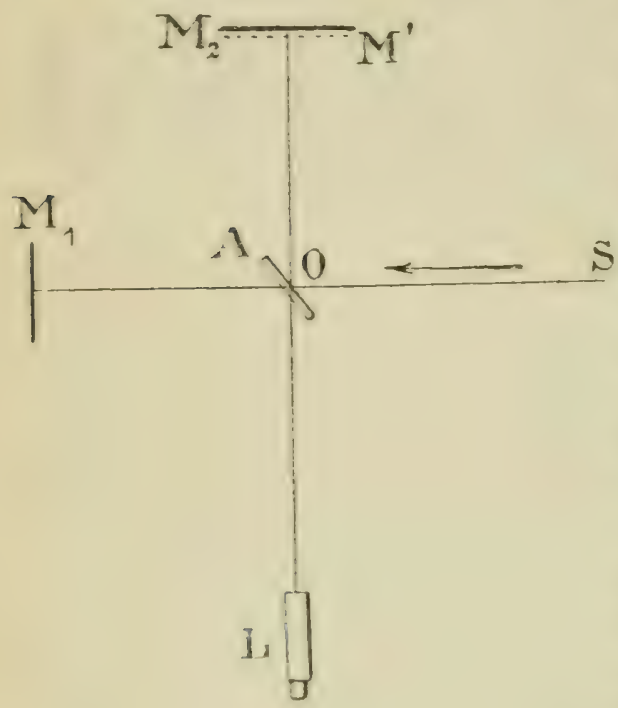

Fig. 6. du miroir Mi produite par la lame argentée $\mathrm{A}$.

Avec ce dispositif, il se produit un phénomène bien connu en optique sous le nom d'interférences lumineuses. Chaque fois que deux faisceaux lumineux issus d'une même source se superposent en se propageant dans une même direction après avoir suivi des chemins différents, on constate des franges, c'est-àdire des lignes alternativement brillantes et obscures dues au fait qu'en certains points les vibrations lumineuses ajoutent leurs effets, alors qu'aux points intermédiaires elles se détruisent mutuellement.

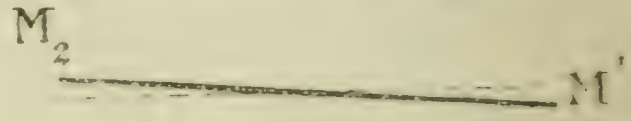

Fic. 7.

Si les deux bras de l'appareil ont la méme longur.u, c'est-à-dire si le miroir Me et le plan de référence M sont superposés, il suffit d’incliner légèrement le miroir $M$. (fig. 7) it de viser ie miroir dans la lunette I, pnur voir des tranges rectilignes (fig. 8). En lumiere monochroma- 
tique (une seule couleur pure), on voit dans tout le champ de: la lunette des franges régulièrement espacées, mais comme l'espacement des franges dépend de la couleur, si l'on emploie de la lumiere blanche on ne voit plus que quelques franges, les autres disparaissant par enchevêtrement des diverses couleurs; ces quelques franges visibles sont irisées, sauf une, la frange centrale qui est noire et nettement reconnaissable.

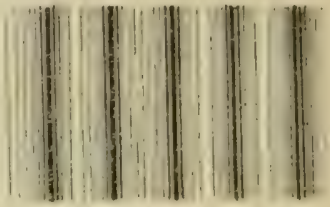

FIG. 8 .

On démontre que cette frange centrale est produite par la superposition de ceux des rayons qui ont mis exactement le même temps à parcourir les deux chemins'.

Si la terre est en mouvement dans l'éther, pour l'obser-



Fic. 9. vateur entraîné avec elle, la vitesse de la lumière doit dépendre de la direction. Considérons de nouveau la surface sphérique sur laquelle doit se trouver un ébranlement lumineux au bout d'une seconde (fig. 9). La terre se trouve en B; par ce point. menons des parallèles aux deux bras de l'appareil : BF et $\mathrm{BG}$ sont les vitesses de la lumière, relativement à l'observateur, suivant les directions des deux bras de l'appareil; ces vitesses étant inégales, la frange centrale, qui correspond aux rayons ayant mis $k$.

1. Ner rouvant faire ici la théorie des interférences, je dois prier le lecteur d'al mettre tous cee ré:ultats. Ici la frange centrale est noire parce que l'un des rasuns $(\mathrm{SO})$ e réléchit en $\mathrm{O}$ en renant de l'air, alors que l'autre rayon $(\mathrm{M}, \mathrm{O})$ e réfléchis en $O$ en venant de l'intérieur du verre. 
même temps à parcourir les deux bras (aller et retour), ne doit pas occuper la position qu'elle aurait si la vitesse était la même suivant les deux bras, et sa position doit dépendre de l'orientation de l'appareil par rapport au mouvement dans l'éther. Par conséquent, si l'on tourne l'appareil (qui est mobile sur une plate-forme), la frange centrale et avec elle tout le système des franges doivent se déplacer par rapport au réticule de la lunette.

Supposons qu'on n'observe aucun déplacement; si l'on considère la théorie précédente comme exacte, on doit penser que B est au centre de la sphère, c'est-à-dire qu à ce moment particulier la terre est immobile dans l'éther, que sa vitesse de translation sur son orbite se trouve, par hasard, compenser exactement la vitesse du système solaire dans l'éther. Mais alors, six mois plus tard, la terre ayant par rapport au soleil une vitesse égale, mais de direction opposée à celle qu'elle avait la première fois, aura, par rapport à l'éther, une vitesse égale au double de sa vitesse orbitale, soit une vitesse de 60 kilomètres par seconde. Pour observer l'effet, on devra placer l'appareil de manière que la différence des temps mis par la lumière à parcourir les deux bras, aller et retour, soit aussi grande que possible, c'est-à-dire orienter l'un des bras dans la direction de la translation de la terre sur son orbite : on observera les franges en les repérant avec le réticule, puis on permutera les rôles des deux bras en faisant tourner la plate-forme d'un angle droit; on devra alors observer un déplacement des franges par rapport à leur position précédente.

L'expérience, faite par M. Michelson en 1881, a été répétée par MM. Michelson et Morley (1887), puis par MM. Morley et Miller (1904-1905) dans des conditions d'extrême précision : par des réflexions successives, le tra- 
jet de la lumière entre la lame et les miroirs avait été porté à 22 mètres. Pour une vitesse de 60 kil. sec., le déplacement des franges, par rotation de l'appareil, devait atteindre une fois et demie la distance séparant deux franges consécutives, valeur énorme car la précision des mesures était du centième de la distance de deux franges.

Fait remarquable: on n'a jamais obtenu aucun déplacement des franges à aucune époque de l'année. Tout se passe comme si la terre élait toujours immobile.

Le désaccord entre l'expérience et la théorie est brutal. Nous allons en chercher les causes.

LA CONTRACTION dE FitZGeraLd-LOREnTZ. - L'expérience de Michelson montre que la lumière met le même temps à parcourir les deux bras de l'appareil (aller et retour) quelle que soit leur orientation. Admettant l'inégalité des vitesses de la lumiere dans la direction de la vitesse de la terre et dans la direction perpendiculaire, on trouve que les deux bras sont parcourus dans des temps égaux sil'on suppose que le bras dirigé dans la direction de la vitesse $v$ de la terre s'est contracté, et que sa longueur, qui serait $l$ si la terre était immobile, est devenue

$$
l^{\prime}=l \sqrt{1-\frac{v^{2}}{c^{2}}},
$$

c étant la vitesse de la lumière dans l'éther. (Appendice, note 3.)

L'hypothèse de M. Fitzgerald et de MI. Lorentz est ainsi la suivante :

Pour tous les corps, les dimensions linéaires parallèles au mouvement dans l'éther subissent un raccourcissement, dû uniquement à ce moulement absolu, dans le rap- 
porl $\frac{l^{\prime}}{l}=\sqrt{1-\frac{v^{2}}{c^{2}}}$. Les dimensions ferpendiculaires ì la vitesse absolue ne sont pas altérées.

Cette contraction serait, en général, très faible icing millioniemes de millimètre par mètre pour une vitesse de 30 kilomitres par seconde) mais elle deviendrait conid rable aux très grandes vitesses, et pour une vitesse eg.ale à la vitesse de la lumière

$$
(v=c \text {, }
$$

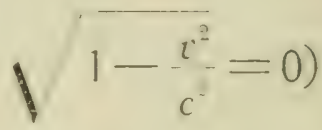

tous les objets seraient réduits à deux dimensions. L'ohservateur ne s'apercevrait d'ailleurs jamais de la contraction, car tous ses instruments de mesure la subiraient, et il la subirait lui-même. Il serait impossible de révéler le mouvement absolu.

Cette hypothèse cherche à sauveoarder les hases de la mécanique classique et la notion de temps absolu dont cll: dérive. La contraction serait bien une contraction réelle produite par le mouvement absolu dans l'ether: elle serait la même pour toute matière.

Mais est-il vraiment possible d'admettre que la contraction, si elle est réelle, soit la méme pour tous les corps. c'est-a-dire soit indépendante de la substance, quelle que soit la rigidité decelle-ci? se produit-elle aussi pour les gar. et alors ou est la limite entre un gaz rarefié et lespace vide?

Comment admettre que la contraction soit une propriéti de la matiere? ne traduirait-elle pas plutôt une proprictí métrique de l'espare dans lequel nous apparait la matice? La théorie d'Einstein nous donnera la réponsc. 
LE. IOINT de VLE d'Einstein. - Pour rendre compte de l'insuccès de toutes les expériences électromagnétiques nu optiques par lesquelles on avait cherché à révéler lo mouvement absolu, pour exprimer les faits de la façon la plus simple, M. Einstein a énoncé les principes suivants :

PRINCIPE DE RELATIVITÉ. - Les lois des phénomines physiques sont les mémes dans tous les siystèmes en translation uniforme les uns par rapport aux autres.

Ce principe constitue l'extension aux phénomènes élcr. Lomagnétiques et optiques du principe de relativité de la mécanique. Sous la forme précédente il est restrcint au cas de la translation uniforme.

PRINCIPE DE LA PROPAGATION ISOTROPE DE: LA LUMIËRE. - Dans tout système en mouvement de Iranslation uniforme (c'est-à-dire dans lequel ne règnc aucun champ de force d'inertie), la vitesse de la lumière cst la même dans toutes les directions; cette vilesse ne dépend pas de l'état de mouvement de la source lumincuse.

Ce principe particulier, conforme au principe de rclativité (restreint), a pour conséquence immédiate que l'expérience de Michelson ne devait rien donner. Le mouvement de la terre sur son orbite peut, pendant la courte durée d'une expérience, être considéré comme rectiligne et uniforme; si la vitesse de la lumière est la même dans toutes les directions, les franges d'interférences gardent évidemment une position invariable quand on tourne la plate-forme de l'appareil de Michelson.

Nous verrons bientôt comment le principe de relativité, joint à la loi de propagation isotrope de la lumière, exige unc transformation radicale des notions d'espace et de temps.

1. Diverses expériences électromagnétiques ont conduit à des résultats neisalifs. 
CHAPITRE III

LINV.ARI ICE DE L.I VITESSE DE L.I LUMIIRE

LE TE.M'S ET IA SIMLITANF́ITE. - M. Einstein a, dés le début de sa théorie. analysé d'une manière remarquable la notion de temps.

On doit d'abord remarquer que, dans toutes les cirronstances où "le temps" joue un rôlc, il s'agit toujours d'événements simultanés. Quand nous disons : le train part à 8 heures, cela signific : l"indication 8 heures des aiguilles de l'horloge et le départ du train sont deux événements simultanés.

La simultanéité de deux événements se produisant au même endroit (ou presque au même endroit) se passe de définition, mais une définition devient nécessaire quand il s'agit de coordonner des événements se produisant en des lieux éloignés.

Considérons un système $\mathrm{S}$ (sans accélération). Au point $A$ se trouvent un observateur et une horloge immobiles dans ce systeme : l'observateur A peut situer dans le temps tous les événements qui se produisent dans son rni. sinage immédiat. En un autre point B, se trouvent aussi un olservateur et une horloge rigoureusement identicue it l'horloge du perint A; cet olservateur B peut, de son coit: ceordonner tous les événements qui se produisent autour 
L.INVARIANCE DE IAA VITESSF. DE, A.A I.LMIERE 35

de lui. La question est de conrdonner les événrmonts qui se passent en $A$ avec ceux qui se passent en $B$, car il s'agit d'avoir, non pas seulement un temps de $\mathrm{A}$ et un tcmps de $\mathrm{B}$, mais un temps commun aux points $\mathrm{A}$ et $\mathrm{B}$.

Ce temps sera défini de la façon suivante:

Puisque, dans un même systène, la vilesse de la lumiene cot la même dans toules les direction. (principe de lisotropie de la propagation), le temps que met la lumière à aller de $A$ en $B$ est égal au temps qu'elle met à aller de $B$ en $A$.

Faisons alors partir de $\mathrm{A}$ un signal lumineux à l'instant l, marqué par l'horloge du point $\mathrm{A}$; ce signal arrive en $\mathrm{B}$ à l'instant $t_{B}$ marqué par l'horloge de $\mathrm{B}$; faisons-le réfléchir sur un miroir placé en $B$ de manière a le renvoyer en $A$; il sera de retour en $\mathrm{A}$ à l'instant $l_{\mathrm{A}}^{\prime}$ marqué par l'horloge de A.

L'horloge du lieu B est synchrone avec celle du lieu A, par définition, si lon a

$$
t_{\mathrm{B}}-t_{\mathrm{A}}=t_{\mathrm{A}}^{\prime}-t_{\mathrm{B}} \quad \text { ou } \quad t_{\mathrm{B}}=\frac{t_{\mathrm{A}}+\frac{1}{2}}{2}
$$

on peut dire encore que l'horloge de B est synchrone ave: l'horloge de A lorsqu'un signal lumincux parti de A à l'époque $t_{A}$ (temps de $\mathrm{A}$ ) arrive en $\mathrm{B}$ à une époqu: $t_{B}$ (temps de B) telle que

$$
\frac{\text { distance } \mathrm{AB}}{t_{\mathrm{B}}-t_{\mathrm{A}}}=\mathrm{c}
$$

c étant la vitesse de la lumière?.

Cette définition du synchronisms ne soulève aurune

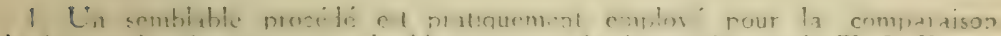

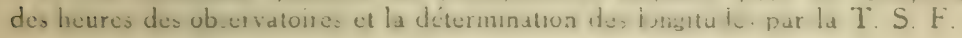


objection, car elle est valable pour tous les points du système de 'référence. En effet :

1. Si l'horloge de B est synchrone avec celle de A. l'horloze de A est synchrone avec celle de B.

2. Si l'horloge de A est synchrone avec l'horloge de B ct arec celle d'un troisieme point $C$, les horloges de $B$ ct de $\mathrm{C}$ sont synchrones entre elles.

Nous comprenons maintenant ce qu'on doit entendre par simultanéité de deux érénements qui se produisent en des licux différents $A$ et $B$. L'événement $E_{\mathrm{A}}$ au point A ct l'événement $E_{3}$ au point $B$ sont simultanés lorsque les cpoques simultanées à ces événements marquées par deux horloges synchrones en A et $B$ sont les mêmes.

Par ces définitions du synchronisme et de la simultaneité, nous avons une définition précise du temps d'un systime Inut entier. Il est essentiel de remarquer yaue cette difinitinn cst baséc sur la propagation isotrope de la lumierc.

Il est impossible de synchroniser deux horlnges en mouvement relatif, car nrus verrons guc deux systèmes cn mouvement l'un par rapport à l'autre ont des temps diffirents.

LA VITESSE DE I.A IUMUERE EST UNE CONSTANTE UNMFRELLE. - Le principe de relativité et le principe de linvariance de la vitesse de la lumiere dans un meme systeme nnt pour conséquence immédiate que la vitesse de la lumière a la nieme ialeur dans bus les systemes an iranslation uniforme los uns par rappont aux autres.

Si, en effel, la vitesse de la lumière devait c̀tre plus grande dans le systeme $S$ que dans le systeme $S$, conmu. toutes les directions de l'espace sont équivalentes dans chague systeme (isotropie) et que rien ne distingue le 
LINVARIANCE DE LA VITESSE DE LA LUMIERE 37

système $\mathrm{S}^{\prime}$ du système $\mathrm{S}$ puisque les lois physiques sont lez mêmes dans ces deux systèmes (principe de relativité), la vitesse de la lumière devrait aussi être plus grande dans $\mathrm{S}$ que dans $\mathrm{S}$; on arriverait à une contradiction. Il faut donc que la vitesse de la lumière soit une constante universelle.

Il est essentiel de bien préciser la signification de ce résultat: nous supposons que, dans divers systèmes en translation uniforme, les observateurs sont munis des mêmes étalons de longueur, c'est-à-dire de règles qui, si on les mettait à côté les unes des autres dans un même système (et bien entendu dans des conditions physiques rigoureusement identiques) auraient la même longueur ; nous sụ̂posons aussi que les observateurs ont des horloges étalonz identiques (appendice, note 4). Dans ces conditions, si ces divers observateurs prennent, chacun dans son système, une base (de longueur et d'orientation quelconque) et mesurent le temps employé par la lumière à parcourir cette base, en divisant le nombre qui mesure la base par le nombre qui mesure l"intervalle de temps, ils doivent tous trouver le même quotient. 


\section{TR.INSFORMIITION DE LORINTZ RELATIVITE DE L'ESP.ACE ET DU TEMPS}

LE GROLPE DE LORENTZ. - On démontre (appendice, note 5) que le principe de relativité et l'invariance de la vitesse de la lumiere conduisent à des formules de transformation de coordonnées profondément différentes de celles de Galité (chap. I, form. 3). Si deux observateurs appartenant à des systemes de référence différents $S$ et $S$ en translation uniforme choisissent un même événement origine et des axes de coordonnées ayant la disposition simple précédemment indiquée (chap). I, fig. 4), les coordonnées d'espace et de temp̣s d'un même événement noté $x, y, z, t$ par l'observaleur du système $S$ et $x, y^{\prime}, z, t$ par l'ohservateur du systeme $S$ doivent être unies par les milations suivantes:

$$
\text { (5) }\left\{\begin{array} { l } 
{ x ^ { \prime } = \frac { 1 } { 2 } ( x - v t ) } \\
{ y ^ { \prime } = y } \\
{ z ^ { \prime } = z } \\
{ i = \frac { 1 } { y } ( 1 - \frac { v x } { c ^ { \prime } } ) }
\end{array} \quad \text { ou (6) } \left\{\begin{array}{l}
x=\frac{1}{\alpha}\left(x^{\prime}+v t^{\prime}\right) \\
y=y^{\prime} \\
z=z^{\prime} \\
t=\frac{1}{3}\left(i+\frac{i x}{c^{\prime}}\right)
\end{array}\right.\right.
$$

v désigne la vilesse du sjstime S' par rapport au systeme $S$ : 
c désigne la vitesse de la lumière; $\%$ représente (abréviation à retenir pour la suite).

Il est essentiel de noter que ces formules sont soumises a la restriction de la relativité restreinte, c'est-à-dire ne sappliquent quà des systèmes en mouvement rectiligne ct uniforme.

Les formules 5 expriment le passage de $\mathrm{S}$ à $\mathrm{S}$ et les formules 6 le passage de $\mathrm{S}^{\prime}$ à $\mathrm{S}$. On voit que les formules 6 ne different des formules 5 que par la permutation des lettres accentuées et des lettres non accentuées et par le remplacement de $v$ par - v; par conséquent, si $v$ est la vitesse de $S^{\prime}$ par rapport à $S$, la vitesse de $S$ par rapport à $\mathrm{S}^{\prime}$ est $-v$.

Le groupe de transformations représenté par les formules qui précèdent a été découvert par M. H.-A. Lorentz, puis retrouvé par M. Einstein comme conséquence des principes qu'il a énoncés. M. Lorentz l'a obtenu en cherchant les conditions pour que les lois générales de l'électromagnétisme, exprimées par les formules de Maxwell, gardent la méme forme dans tous les systèmes de référence (en translation uniforme), c'est-à-dire soient les mêmes dans wus les systemes, condition nécessaire pour qu'elles aient une réalité indépendante de l'observateur. M. Lorentz a établi :

$I^{\prime \prime}$ que les équations fondamentales de l'électromagnétisme nadmettent pas le groupe de transformations de la méc arique (groupe de Galilée), c'est-à-dire qu'en effectuant dans ces équations les transformations de ce groupe, on obtient des équations d'une forme tout à fait différente.

2 "que ces équations admettent un autre groupe de transformations, celui exprimé par les formules 5 et 6 . 
La différence entre le groupe de Lorentz et celui de Galilée est profonde. Au licu du temps $t$ du système S, il fant introduire dans le système $S^{\prime}$ un autre iemps $i$ que M. Lorentz a appelé temps local (parce qu'il dépend de la coordonnée $x$ du lieu considéré).

M. Lorentz avait considéré ce temps local comme une fiction mathématique. Il appartient à M. Einstein de lui avoir attribué une réalité physique : c'est le temps que marquent, dans le système $\mathrm{S}^{\prime}$, des horloges identiques ì celles qui, dans le système $\mathrm{S}$, mesurent le temps $t$.

LES LOIS DE LA MÉCANIQLE DOIVENT ÊTRE. CONPATIBLES AVEC CELLES DE L'ÉLECTROMAGNÉTISML. En résumé, les deux principes énoncés par $M$. Einstein (chap. II), le principe de relativité et le principe de l'isotropie de la propagation de la lumière ont pour conséquence :

$I^{\prime \prime}$ que la vitesse de la lumière est une constante universelle;

$2^{\prime \prime}$ que les transformations des coordonnées d'espace et de temps, quand on passe d'un système à un autre (sous la réserve de la translation uniforme) sont les transformations du groupe de Lorentz. On pcut vérifier que ces transformations conservent leur structure aux équations du champ électromagnétique.

Inversement, sil'on cherche, comme l'avait fait M. Lorentz, les formules de transformation qui laissent invariantes les lois de l'électromagnétisme, on obtient les formules (5) et (6) qui impliquent la constance de la vitesse de la lumière et la relativité du temps.

Nous sommes donc en présence de deux groupes de. transformations : 
1" Le groupe de Galilée, qui seul laisse invariantes les lois de la mécanique classique;

2" Le groupe de Lorentz, qui seul laisse invariantes les lois de l'électromagnétisme.

Doit-on conserver à la fois les lois de la mécanique classique avec le groupe de Galilée, et les lois de l'électromagnétisme avec le groupe de Lorentz?

Cela est impossible. Les premières admettent un temps absolu, les secondes impliquent un temps relatif : Adopter le temps absolu de la mécanique, c'est renoncer à l'invariance des lois de l'électromagnétisme; adopter le temps relatif de l'électromagnétisme, c'est abandonner la mécanique newtonienne. Il y a bien incompatibilité radicale, car il n'y a qu'un seul temps physique dans un même système de référence.

Le désaccord qui s'est manifesté entre la théorie mécanique de l'expérience de Michelson et le résultat expérimental apparaît comme la cause d'un conflit entre les lois de la mécanique classique et celles de l'électromagnétisme.

Il faut choisir, et il n'est pas permis d'hésiter, puisque le choix est imposé par l'expérience : les lois de l'électromagnétisme sont trop bien vérifiées pour qu'on puisse sonser à les abandonner; l'expérience est d'accord avec le groupe de Lorentz qui exprime l'invariance de ces lois. Cela est d'ailleurs logique et l'on devait s'y attendre : les lois de l'électromagnétisme ont été établies dans un système de référence qui n’est nullement privilégié dans l'univers; elles s'expriment sous une forme claire et simple, et deviendraient compliquées par une transformation différente de celle du groupe de Lorentz. Il serait déraisonnable de supposer que ces lois simples sont spéciales à un système de référence lié à la terre et d'ailleurs la preuve de leur inva- 
riance est le fait qu' elles ne changent pas dans le cours de l'année, malgré le changement du système de référence, la terre changeant de direction sur son orbite.

$\mathrm{Au}$ contraire, nous n'avons aucune raison de considérer les lois de la mécanique comme exactes; elles peuvent paraitre valables dans les phénomènes ordinaires, trop grossiers pour qu'une discordance se révèle, mais dès quil s'agit de phénomènes comportant, comme l'expérience de Michelson, une vérification d'une haute précision, le désaccord apparaît.

Ainsi, le résultat de Michelson, l'échec de toutes les tentatives faites pour révéler le mouvement absolu de la terre, tiennent à des causes profondes, qu'on n'avait pas soupçonnées dans les débuts de la théorie électromagnétique, mais qu'on s'explique aujourd'hui. Il faut renoncer à considérer les lois de la mécanique classique comme des lois rigourcuses; il faut soumetire la mécanique aux lois deléleciromagnétisme, en appliquant à tous les phénomènes les formules de transformation d'espace et de temps du groupe de Lorentz. Les lois classiques deviennent alors des approximations, d'ailleurs excellentes dans la plupart des cas : on remarque, en effet, que si la vitesse de la lumiere était infinie, on aurait les formules de Galilée. Or la vitesse de la lumière est très grande, et tant que le carré de la vitesse des corps (vitesse par rapport à l'observateur) peut étre négligé vis-à-vis du carré de la vitesse de la lumière, on peut se servir de la mécanique habituelle.

On voit, par cette dernière remarque, que le desaccord entre la mécanique newtonienne et l'électron agnétisme est un aspect du conflit profond qui a dominé la physique jus. qu" à l'époque actuelle : le conflit entre la thérie des aclimins it distance instantances admisce en méranique cóbste jus- 
quà la découverte de la loi nouvelle de la gravitation (loi d'Einstein), et la théorie de l'action de proche en proche aiec vitesse finie, à laquelle Maxwell a donné son plein développement.

Les équations de Maxwell entraínent la négation du temps physique absolu; impliquant la notion de temps relatif, ces équations interdisent la possibilité d'une relation de cause à effet, quelle qu'elle soit, pouvant se propager avec une vitesse infinie.

Nous affirmons donc que la seule cinématique ayant un sens expérimental et aussi grâce à laquelle les lois de la physique prennent une forme simple, indépendante du sysième de référence, est la cinématique du groupe de Lorentz. (M.-P. Langevin. ${ }^{1}$ )

C"est là la base solide de la théorie de la relativité et de la mécanique nouvelle.

L'esp.ACE et le temps Relatifs, - Avec les formules de Lorentz, oì le temps n'est plus un invariant, nous voyons disparaitre la dissymétrie qui, avec le groupe de Galilée, existait entre l'espace et le temps. Dans l'ancienne cinématique, la distance spatiale de deux événements non simultanés dépendait du système de référence, mais l'intervalle de temps écoulé entre eux était absolu. Maintenant, la durée écoulée est relative, tout cómme l'intervalle d'espace. Soient, en effet, deux événements (indices 1 ct 2); les formules de Lorentz (éq. 5) donnent :

(7)

$$
\begin{aligned}
& \mid x_{2}-x_{1}=\frac{1}{\%}\left(x_{2}-x_{1}\right)-\frac{1}{x} v\left(t_{2}-t_{1}\right) \\
& \mid t_{1}-t_{1}=\frac{1}{x}\left(t_{2}-t_{1}\right)-\frac{1}{x} \frac{v}{c^{2}}\left(x_{2}-x_{1}\right)
\end{aligned}
$$

1. Bulletin de la sociélé des électriciens, $n^{\circ} 84$, déc. 1919. 
La symétrie de ces deux équations est remarquable. Il n'y a plus de simultanéité absolue, car lorsque deux événements sont simultanés dans un système $\left(t_{1}=t_{2}\right)$ ils ne sont simultanés dans aucun autre système en mouvement par rapport au premier (puisque d’après la seconde équation (7) $t_{1}^{\prime}$ est différent de $t_{2}$ ), à moins que ces événements ne coïncident à la fois dans l'espace et dans le temps. Dans ce dernier cas, la coïncidence a lieu dans tout système, il y a coïncidence absolue. On comprend aisément que la coïncidence dans l'espace et dans le temps ait un sens absolu, car il peut en résulter un effet sur lequel tous les observateurs sont nécessairement d'accord (par exemple rupture de deux objets par choc mutuel).

La relativité complète de l'espace et du temps perçus par chaque observateur entraine la suppression des notions de systeme fixe et de mouvement de translation absolu. L'éther, du moins celui admis autrefois, doué de propriétés élastiques et mécaniques, doit être supprimé. Nous verrons. dans la relativité généralisée, par quelle conception on peut le remplacer.

LA COMPOSITION DES VITESSFS. - Un ohservateur (système $\mathrm{S}$ ) voit passer un train avec une vitesse $\iota$, dans le train (système $S^{\prime}$ ) un homme se déplace avec hr vitesse $i^{\prime}$ (par rapport au train), quelle est la vitesse de $\quad \cdots$ homme par rapport a l'observateur?

On est tenté de répondre $v+i$. C'est en effet la lin de composition des vitesses qui résulte de l'ancienne cinćmatique.

Cela parait évident, parce quil est difficile de se délnrrasser des anciennes notions d'espace et de temps. Cependant il résulte des formules de Lorentz (appendice, note 6) 
que la vitesse $u^{\prime \prime}$ du mobile, mesurée dans le système $\mathrm{S}$, est, non pas $v+v^{\prime}$, mais

$$
v=\frac{v+\frac{v^{\prime}}{1+\frac{v v}{c^{2}}}}{}
$$

Cette formule s'applique à la composition de deux: vitesses mesuréc:s dans des sustèmes différents (o est mesurée dans le système $\mathrm{S}, i$ est mesurée dans le système $S^{\prime}$ ).

Evidemment, dans l'exemple du train, le terme $\frac{v^{\prime \prime}}{c^{2}}$ qui intervient au dénominateur est absolument négligeable, de snite que la loi ancienne est une approximation plus que suffisante: mais il n'en serait plus de même si les ritesscs étaient considérables : supposons un observateur $\mathrm{A}$ et deux ohservatcurs $B$ et $C$ s'éloignant de $A$, dans des directions npposécs, avec la vitesse, mesurée par A, de 200000 kilomètres par seconde. Pour l'observateur $\mathrm{A}$, les observateurs B et C s'éloignent l'un de l'autre de 400000 kilomètres par seconde - ceci reste exact, bien entendu - mais si chacun des observateurs $\mathrm{B}$ et $\mathrm{C}$ mesurait la vitesse de lautre, il trouverait seulement 277000 kilomètres par seconde.

La nouvelle loi de composition des vitesses montre qu' un mobile, par accroissements successifs de vitesse à partir de sa vitesse primitivement acquise, n'atteint jamais la vitessc $c$ de la lumière. La vitesse de la lumière est une vilesse limile qui ne peut être dépassée, et si l'une des vilesses v ou v' était égale à c, on trouverait encore $v=c$.

L'extérience de Fizeau, dite “ entrainement 
DES ONDES I UMINFUSES I UR I. I MATIERE FN MOUTE. MENT". - Une célébre expérience, réalisée en $18 j 1$ par Fizeau, vérific remarquablement bien la nouvelle loi de composition des vitesses.

On sait que dans la matiere au repos (par rapport a l'olservateur) la vitesse de la lumière est ${ }^{c} n$ étant lindice de réfraction de la matiere, variable avec la radiation employée!.

Quelle est, pour l'observateur, la vitesse de la lumière dans un milieu animé d'une vitesse $t$ ? Fresncl a déduit de considérations théoriques que ectte vitesse doit être

$$
\frac{c}{n}-c\left(1-\frac{1}{n^{2}}\right)
$$

la "ritesse d'entrainement " $i\left(1-\frac{1}{n^{2}}\right)$ s.joutant à i.a vitesse $\frac{c}{n}$ dans la matiere au repos ou se retranchme de cette vitesse selon que le sens du mourement de la matiore est celui de la propagation de la lumiere ou le suns opposé.

La formule de Fresnel a ćté véritiée par Fizeau. Ce physicien a observé les franges d'interférences produites par deux rayons issus d'une même source, après prassage en des sens opposés dans des tubes remplis d'eau, et a déduit de-s mesures du déplacement des franges, Inreque l'eau est en mourment, que $l e$ "coeflicient d'entrainement" cas bien $\left(1-\frac{1}{n^{2}}\right)$.



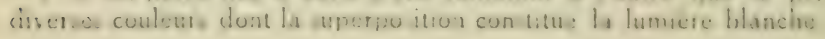


Ce résultat arat été interprété en admettant un entrainement de l'éther, non pas trial, mais partiel /avec une vitesse $v\left(1-\frac{1}{n^{2}}\right) \mid$, interprétation étrange car l'entrainement de l'éther dépendrait de $i$, c'est-à-dire dépendrait de la coulcur de la radiation employée.

Cette loi d'entraincment s'explique immédiatement, de la façon la plus simple, par la cinématique nouvelle.

Il suffit d'écrire la loi de composition des vitesses (8). Le courant d'eau (système $\mathrm{S}$ ) coule relativement à l'oljservateur (systeme S) avec la vitesse $i$. Le rayon lumincux se propage dans l'cau (systicme S') arec la vitesse

$$
v^{\prime}=\frac{c}{n}
$$

la vitesse :" de ce rayon mesurée par l'abservateur est donc, d'après (8),

$$
v^{\prime \prime}=\frac{v+v^{\prime}}{1+\frac{v v^{\prime}}{c^{2}}}=\frac{v+\frac{c}{n}}{1+\frac{v}{c n}}
$$

$=$ approximativement $\frac{c}{n}+c\left(1-\frac{1}{n^{i}}\right)$

C'est bien le résuliat vérifié par Fizeau.

Il convient de mentionner que M. Lorentz arait expliqué ce phénomène par l'action des électrons entrainés avec la matiere, mais cette explication, basée sur les lois de l'́lcclromergiélisme, n'est au fond qu'une forme déguiséc de l'explication relativiste. 


\section{CIIAPTRE V \\ L'UNIVERS DE MINKOWSKI}

- A l'heure actuelle, l'espace et le temps considérés en eux-mêmes doivent disparaitre comme des fantômes et seule leur union peut posséder une individualité

H. Minkowski

(Raum und Zcil, 1908.)

UNION DE L'ESPACE ET DE TEMIS. - Snient deux événements quelconques. Lorsqu'on les repère dans des systemes différents, la duréc $T$ qui les sépare et la distance spatiale I des points cu ils se produisent varient din sy-stème à l'autre, mais la quantité

$$
s^{2}=c^{2} T^{2}-l^{2}
$$

a la même valeur dans tous les systemes; on le viritic immédiatement en appliquant les formules de Lorentr.

En langage ordinaire, le carré du produit de la vitessee de la lumière par le temps écoulé entre les événements, diminué du carré de leur distance dans l'espace, est une quantité indépendante de tout système de référence ( $\mathrm{cn}$ translation uniforme).

$$
\text { LINVARIANT \& EST LINTERV.ILLE DOUIVERS. }
$$
il vient remplacer les deux invariants d'autrelois (chap. 1): le temps et la distance dins l'espace de deux érénements simultanés.

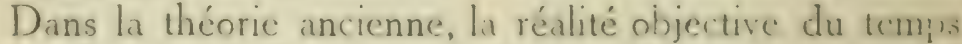
était affirmée par l'invariance du temps (le temps universcl 
ct absolu); la réalité objective de l'espace résultait de l'invariance de la distance géométrique de deux points (distance de deux événements simultanés).

Il n'y a plus maintenant d'espace absolu ni de temps absolu; il ne subsiste qu'une réa!ité unique affirmée par l'invariant $s$. La modification est radicale: le nouvel invariant contient à la fois les trois coordonnées d'espace $x, y$, zet la coordonnée de temps $t$
$(10)$
$s^{2}=c^{-}(t=$
$\left.t_{i}\right)^{2}$
$-\left(x_{2}-x_{1}\right)^{-2}$
$-\left(y_{2}-y_{i}\right)^{2}-\left(z_{1}-z_{1}\right)^{2}$

L'espaze et le temps, unis par cet intariant, ne sont pas indépendants et leur union seule possède une individualité. L'Espace-Temps ou Univers est l'ensemble des événements ; c'est une multiplicité "quadridimensionnelle".

L'Univers est indépendant du système de référence qui sert à repérer les érénements; chaque système est une division particulière de l'Univers en espace et en temps.

L'espace reste toujours l'ensemble des événements simultanés; c'est une " coupe de l'Univers à temps donné (P. Langevin). Cette définition s'applique à l'ancienne conception de l'espace et à celle d'aujourd'hui, mais la différence est profonde: la conception compatible avec la mécanique nevtonienne admettait un temps universel, et la coupe était la même pour tous les systemes, il n'y avait qu'une division de l'Univers en espace et en temps - d'où la possibilité d'envisager séparément l'espace et le temps la forme des corps était la même pour tous les observateurs.

Dans l'Univers de Minkowski, la simultanéité étant relative, la coupe à temps donné dépend du systène de reférence : la forme des corps niest plus intariable, il y a une infunité d'espaces euclidiens dans l'L'aivers "eic'idien" 
unique à quatre dimensions (comme en génmétric il y a unc infinité de plans dans l'espace euclidien à trois dimensions).

PROPRIÉTÉS DES COURIES D'FYÉNEMENTS (P. LANGeviN). - Soicnt A et B deux érénements. Triş cas peuvent se présenter, le carré $s^{\prime \prime}$ de l'interyalle cst négatif, nul, ou positif.

I" COL PLIES D.INS LLSP.ICE. - Si $s^{2}$ est négatif, cola veut dire : dans tous les systemes de référence, la disLance $l$ des points oì se produisent ces événements est plus grande que le trajet $c T$ que parcourt la lumière dans l'intervalle de temps qui les sépare.

Une application simple des formules de Lorentz permut d'étahlir ģue deux tels événements nont pas un ordre de succession déierminé. Il existe une infinité de systemes de référence dans lesquels $\mathrm{A}$ est antérieur à $\mathrm{B}$, une infinité de systèmes dans lesquels A cst, au contraire, postéricur à $B$, enfin un systeme dans lequel $A$ et $B$ sont simultanés.

La distance spatiale de ces deux ciénements esl minimum dans le système pour lequel ils soni simultanés car $s^{2}=c^{2} \mathrm{~T}^{-}-l^{2}$ étant constant, $l^{2}$ est minimun lorsque $\mathrm{T}$ est nul.

Deux tels événements qui, par un choix convenable de la division de l'Univers en espace et en temps peuvent êtie amenés en cö̈ncidence dans le temps, mais jamais dans l'espace, forment un couple d'érénements dans l'espare.

Deux érénements constituant un couple dans liespace sont absolument indépendants, car s'il existait entre cux un lien de cause à effet, comme leur ordre de succession nicst pas déterminé, la cause serait, pour certains observateurs, postéricure à leffet, ee qui est alssurde : comme dit 
M. Einstcin " on ne peut pas télégraphier dans le passé ". 2" COINCIDENCE ABSOLLE. - Lorsque s est nul, on a dans tous les systèmes $l=c T$; c'est le cas qui sc présente pour deux "points d'Univers" d'un rayon lumincux, puisque le trajet $l$ parcouru par la lumière (dans le vide) pendant le temps $T$ est précisément $c T$. Dans le cas où $l$ ct $T$ sont nuls tous deux dans un systeme, ils sont nuls dans tous les systèmes; les deux événements sont en coïncidence absolue.

$3^{\prime \prime}$ COUPLES D.INS LE TEMPS. - Lorsque l'invariant $s^{2}$ est positif, la distance spatiale $l$ est, dans tous les systèmes de référence, plus courte que le trajet $c \mathrm{~T}$ de la lumière pendant la durée écoulée entre les deux événcments. Le calcul montre que l'ordre de succession des deux ćénements considérés a un sens bien délerminé. On ne peut jamais les rendre simultanés, c'est-à-dire trouver un système de référence pour lequel ils soient en coïncidence dans le temps; mais on peut les amener en coïncidence dans l'espace, et la ciurće $\mathrm{T}$ qui les sépare est minimum danns le syslème pour lequel ils coüncident dans l'espace.

Deux événcments pour lesquels $s^{2}$ est positif forment un couple dans le temps. Ils peuvent être unis par un lien de causalité; ils peuvent aussi, bien entendu, être indépendants, mais toujours le premier événement a pu être annoncé au licu où le second va se produire, puisque la distance spatiale qui les sépare est, dans tous les systèmes, plus courte que le trajet $c \mathrm{~T}$ que parcourt, dans le temps $\mathrm{T}$, un signal lumineux ou électromagnétique.

L'invariant $\mathrm{s}^{2}$ est donc positif ou négatif suivant qu'un des érénements peut ou non influer sur l'autre; il indigue la "possibilité d'influence ou d'action " d'un des événements sur l'autre (M. P. Langevin). 
LA CONTRACTION DES LONGLEURS. - Dans deux systèmes de référence en mouvement relatif, prenons des axes ayant la disposition simple que nous avons adoptée (fig. 4). Imaginons une tige, parallèle aux axes $\mathrm{O} x, \mathrm{O}^{\prime} x$, immobile dans le système $\mathrm{S}$ et par conséquent se déplaçant, dans le systeme $\mathrm{S}$, avec la vitesse $v$ dans le sens de sa longueur.

Prenons comme événements $\mathrm{A}$ et $\mathrm{B}$ les positions des extrémités de la tige à un même instant pour l'observateur du système $\mathrm{S}$; ces deux événements étant en coïncidence dans le temps pour le système $\mathrm{S}$ forment, d'après ce qui a été dit plus haut, un couple dans l'espace, et leur distance spatiale est minimum dans le système $\mathrm{S}$ où ils sont simultanés.

Pour l'observateur du système $\mathrm{S}$, la distance spatiale des positions simultanées des extrémités de la tige est la longueur de cette tige; la tige est donc plus courte pour l'observateur du système $\mathrm{S}$ que pour l'observateur du système $\mathrm{S}$ pour qui les événements $A$ et $B$ ne sont plus simultanés.

Ainsi, pour l'observateur $\mathrm{S}$ qui voit passer la tige, celleci est plus courte que pour l'observateur $S^{\prime}$ pour quil la tige est immobile; il est facile de calculer (appendice, note 7) que le rapport entre les longueurs de la tige animée de la vitesse $v$ et de la même tige au repos est $\sqrt{1-\frac{v^{2}}{c^{2}}}$.

C'est précisément la contraction de Fitzgerald-Lorentz (chap. II) mais ici cette contraction n'a plus aucun caractère absolu, et elle ne prête plus aux objections que nous avons faites. En somme, elle résulte simplement de la manière différente dont les deux olsservateurs envisagent la simultanéité, et du fait que la forme d'un corps en nouvement ne peut être définie que comme l'ensemble des positions simultancés des différents points de ce corps. 
La contraction est tellement peu absolue, quelle est réciproque, c'est-à-dire que si deux tiges identiques sont immobiles, l'une dans le systèrne Sl'autre dans le système S', chaque observateur estime que la tige de l'autre systène est plus courte que celle de son système.

Le fait qu'un objet en mouvement est contracté dans le sens du mouvement ne signifie donc pas que l'objet a été récllement modifié par le mouvement; il signifie qu'un observateur lié à l'objet et un observateur en mouvement par rapport à l'objet ne font pas la même décomposition de l'Univers en espace et en temps, que l'espace relatif à l'objet et l'espace relatif à l'observateur qui le voit passer ne sont pas les mêmes (ainsi que nous l'avions fait pressentir, page 32).

LA Dilatation du temips (EINStein). - La contraction des longueurs a une contre-partie, la dilatation du temps. Le calcul montre (appendice, note 7) que, pour les observateurs immobiles dans un des systèmes $\mathrm{S}$ ou $\mathrm{S}^{\prime}$, les horloges de l'autre système retardent: chaque observateur, dans son systeme, divise par $\sqrt{1-\frac{v^{2}}{c^{2}}}$ les intervalles de temps mesurés par une horloge au repos dans l'autre système.

Les ligNes d'Livers (MiNkowski). - Suivons maintenant la succession continue des événements qui constituent la vie d'une même portion de matière ou d'un même être. Leur ensemble forme dans l'Espace-Temps une ligne dUnivers, comme en géométrie une succession continue de points forme une ligne dans l'espace.

En géométrie, pour mesurer un arc de courbe $A B$, cn décompose cet arc en cordes rectilignes très petites, et lion 
fait la somme de ces petites cordes $\mathrm{A} a, a b, b c$, etc... (fig. 10); plus les cordes sont petites



A

FIG. 10 (et en même temps, bien entendu, plus grand est leur nombre), plus la somme de leurs longueurs est voisine de la longueur de l'arc de courbe, ce qu'on exprime en disant que la longueur de l'arc $\mathrm{AB}$ est l'intégrale, prise de $\mathrm{A}$ à $\mathrm{B}$, des cordes infiniment petites. On a l'habitude de désigner une intégrale ou sommation de quantités infiniment petites (en nombre

infini) par le signe $\int^{\circ}$, et l'on écrit

$$
\operatorname{arc} \mathrm{AB}=\int_{\mathrm{A}}^{\mathrm{B}} \mathrm{d} l
$$

cn désignant par $d l$ l'une quelconque des cordes infiniment petites (1), ou ce qui revient au même, un arc de courhe ćlémentaire, car l'arc de courbe et la corde rectiligne entre deux points tendent à avoir la même longueur si les deux points se rapprochent indéfiniment. Ainsi, il est bien entendu que le symbole $\int_{A}^{B} d l$ signifie la somme des cordes infiniment petites, ou ce qui est la même chose la somme des arcs de courbe élémentaires, depuis le point $\mathrm{A}$ jusqua au point B.

Opérons de la même manière pour une ligne d'Univers

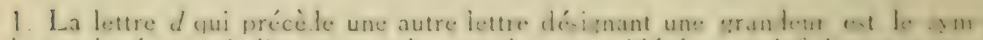

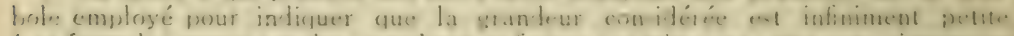



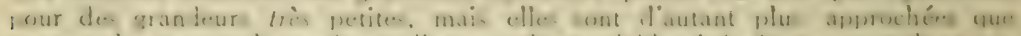


deurs infiniment petites. 
quadridimensionnelle : entre deux points-événements $\mathrm{A}$ et $\mathrm{B}$ de cette ligne, nous décomposons la succession continue d'événements en "intervalles" ds infiniment petits, dans chacun desquels le mouvement de la portion de matière envisıgée peut être considéré comme rectiligne et uniforme (de même qu'en géométrie chaque arc élémentaire peut être confondu avec la corde rectiligne).

D'après ce que nous avons vu au début de ce chapitre, chacun de ces intervalles élémentaires est un invariant icomme en géométrie la longueur des cordes infiniment pelites est indépendante du système de coordonnées). La longueur de l'arc de ligne d'univers, qui est la somme des intervalles infiniment petits, c'est-à-dire l’intégrale

$$
I=\int_{\mathrm{A}}^{\mathrm{B}} d s
$$

étendue à tous les couples d'événements infiniment voisins qui se succèdent d'une manière continue le long de la ligne d'Univers, a donc une valeur indépendante du système de référence.

Prenons comme système de référence un système lié à la portion de matière considérée : dans ce système, tous les événements concernant cette portion de matière sont fixes dans l'espace, puisqu'ils occupent la méme position par rapport aux axes du systeme; donc, puisqu'on peut les amener en coïncidence dans l'espace, pris deux à deux ils constituent des couples dans le temps. Par suite leur ordre de succession ne peut être inversé : le passé, le présent et l'avenir gardent un ordre immuable pour les événements concernant un même objet ou un même être.

LE. TEMPS I'ROPRE (MINKOW'NI), - Sur la ligne d'Uni- 
vers d'une portion de matière, choisissons deux événements infiniment voisins, séparés par un intervalle d'Univers $d s$ (infiniment petit); soient $d l$ leur distance spatiale (infiniment petite) et dt l'intervalle de temps (infiniment court) qui s'écoule entre eux, dans un système de référence quelconque. Nous avons, d'après la définition même de l'intervalle (éq. 9),

$$
\text { (12) } \quad d s^{2}=c^{2} d t^{2}-d l^{2}=\text { invariant. }
$$

Dans le système de référence lié à la portion de matière, $d l$ est nul; soit $d$. l'intervalle de temps dans ce sy3tème

$$
d s^{2}=c^{2} d t^{2} \quad \text { ou } \quad d s=c d
$$

et, par intégration entre deux événements $\mathrm{A}$ et $\mathrm{B}$ quelconques pris sur la ligne d'Univers

$$
\text { (13) arc de ligne d'Univers }=\int_{A}^{D_{B}} d s=c \int_{A}^{{ }^{B}} d=
$$

d: est l'élément de temps propre de la portion de matière considérée et de tout le système qui lui est lié. Le temps propre total $\int_{\mathrm{A}}^{\mathrm{B}} d$ - écoulé entre deux événements $\mathrm{A}$ et $\mathrm{B}$ est le temps que mesurera un observateur. c'est le temps qu'enregistreront les horloges dans ce système. Ce temps propre est indépendant de tout système de référence.

Ainsi une horloge liéce à un mobile (dont le mouvement n'a plus besoin ici d'être soumis à la restriction dela translation uniforme) mesure la longueur, divisée par c. de lare de ligne d'Univers de ce mobile.

Nous avons vu que lorsque deux événements forment un couple dans le temịs, la durée qui les sépare est mini- 
mum dans le système pour lequel ils sont en coïncidence dans l'espace; le temps propre jouit donc de cette propriété de minimum, il est plus court que le temps évalué dans tout systeme en translation uniforme.

On démontre (appendice, note 8) que si un mobile est anim forme, l'élément de temps propre d' écoulé entre deux événements infiniment voisins pris sur sa ligne d'Univers est lié à l'élément de temps $d t$ mesuré entre les deux mêmes événements dans le système $\mathrm{S}$, par la relation

$$
\text { (14) } \quad d z=r d t, \quad\left(x=\sqrt{1-\frac{v^{2}}{c^{2}}}\right) .
$$

Le coefficient $\%$ est d'autant plus petit que la vitesse $v$ est plus voisine de la vitesse de la lumière. Le temps propre est done d'autant plus court (par rapport aut temps du systeme $\mathrm{S}$ en translation uniforme) que la vitesse du mobile dans le système $\mathrm{S}$ est plus grande.

On démontre encore (note 8) qu entre deux événements déterminés, la plus longue ligne d'Univers est celle qui correspond au mouvement rectiligne et uniforme. Il n'y a pas de ligne de plus courte distance, mais il existe une infi. nité de lignes d'Univers de longueur nulle, qui correspondent à toutes les trajectoires imaginables des rayons lumineux entre les deux événements (pour un rayon lumincux, on a toujours $d l=c d t$ et par conséquent $d s=0$ ).

D'étranges conséquences se déduisent de ces résultats.

1" Dans un système en translation uniforme - la terre par exemple, car son accélération est négligeable - deux hor!oges identiques et synchrones sont au même endroit. On déplace l'une très rapidement et on la ramène près de l'autre au bout du temps $t$ (temps du système); le temps propre de 
I'horloge qu'on a déplacée, à laquelle on a fait subir une accélération, ayant été plus court que le temps du système uniforme, cette horloge se trouve en retard sur l'autre horloge, de $t-\int_{0}^{t} \% d t$. C'est l'accélération qui a créé la dissymétrie; on reconnait ici le caractère absolu de l'accélération signalé à la fin du chapitre I.

2" Dans les mêmes conditions, un échantillon de matière radioactive aura moins évolué que celui qui n’a pas été déplacé, qui n’a pas subi d'accélérations (M. Langevin).

3" Avec M. Langevin, imaginons qu'un observateur ait une machine lui permettant de quitter la terre et d'atteindre une vitesse fantastique. Supposons, pour fixer les idées, que cette vitesse soit inférieure de $\frac{1}{20000}$ seulement à la vitesse de la lumière. Pendant un an, le voyageur séloigne de la terre et il revient au bout de deux ans: il n'a vicilli que de deux ans, car il a vécu le temps propre de son système', temps enregistré par ses horloges. Cependant, à son retour, il trouve sur la terre d'autres générations, et il apprend qu il est parti depuis 200 ans. Il s'est transporté daris l'avenir de la terre, mais sans retour possible dans le passé.

Ces chiffres supposent que la vitesse a étés atteinte très rapidement, ce qui serait évidemment impossible, méme si l'homme disposait d'une énergie suffisante, car la force d'incrtie due à laccélération serait telle que le inyageur

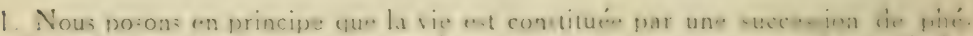

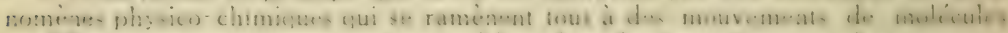

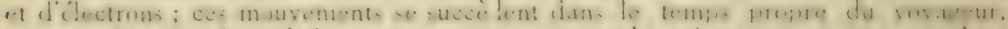

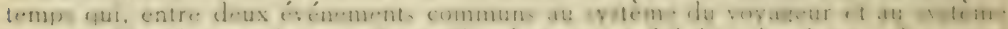

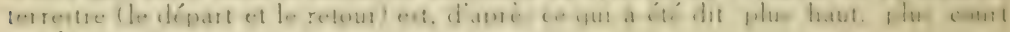
que le temps terrestre. 
serait écrasé. Toutefois, cet exemple met admirablement en évidence la relativité du temps.

Pour un mobile qui serait animá de la vitesse de la lumière (c'est-à-dire dont la ligne d'Univers serait de longueur nulle), le cours du temps serait suspendu.

LA LoI D’iNertie. - Nous avons déjà, au chapitre I, énoncé la loi d'inertie de Galilée : un mobile libre est animé d'un mouvement rectiligne et uniforme. D'autre part, nous venons de voir que la ligne d'Univers la plus longue entre deux événements déterminés est celle qui correspond à un mobile allant d'un événement à l'autre d'un mouvement rectiligne et uniforme. Nous pouvons donc donner à la loi de Galilée la forme suivante : entre deux événements concernant un mobile sur lequel n'est appliquée aucune force, la ligne d'Univers la plus longue est précisément la ligne d'Univers de ce mobile; ou encore, la loi d'inertic est la loi du temps propre maximum.

Le mouvement rectiligne et uniforme joue, dans l'Univers de Minkowski, le rôle que joue la ligne droite en géométrie euclidienne, avec cette différence que la ligne d'Univers qui se traduit à nous par ce que nous appelons l'état de mouvement rectiligne et uniforme entre deux événements est la ligne d'Univers la plus longue, alors qu'en géométrie la ligne droite tracée entre deux points est la ligne la plus courte. Cependant, dans un cas commedans l'autre, on peut donner un même énoncé (voir appendice, note 9) et la ligne du point matériel libre, dans un Unicers régi par les formules de Lorentz, peut être qualifiée de droile d'Univers, car elle présente une analogie frappante avec la droite de la géométrie euclidienne. 


\section{DYNAMIQUE DE LA RELATIVITÉ}

A la cinématique définie par le groupe de transformations de Lorentz, qui remplace la cinématique ancienne basée sur le groupe de Galilée, corresıond une dynamique nouvelle, qui, fait remarquable, est plus cohérente et plus simple que la dynamique newtonienne. Nous nous bornerons ici à indiquer les résultats. Un aperçu général de la thénrie est donné dans la note 10 de l'appendice.

LA MASSE FONCTION DE LA VITFSSE. - Dans la dynamique ancienne, la masse newtonienne d'une portion de matière est une grandeur invariable (chap. 1). Dans la dynamique nouvelle, deux olsservateurs en mouvement l'un par rapport à l'autre ne doivent pas aitribuer la même masst' à une méme portion de matière. La masse d'un corps est relative comme sa vitesse, et dans un système de référence déterminé, la masse augmente avec la vitesse.

Si l'on conserve la défnition de la masse (coefficient d'inertie) donnée au chapitre 1, on trouve quil faut envisager deux masses: une masse longitudinale, qui intervient si la forre agissante (et par suite l'accélération) est dirigée parallèlement à la vitesse acquise: une masse transversale dans le cas où la force agit normalement à la trajectoire: ces deux masses augmentent avec la vitesse de la portion de maticre 
considérée, mais suivant des lois différentes; elles ne sont égales que si la portion de matière est au repos.

Une autre définition de la masse permet de ne conserver qu'une seule masse. Supposons qu'une force F constante agisse pendant un temps $t$ sur une portion déterminée de matière, initialement au repos; au bout du temps $t$ cette force a imprimé à la matiere une vitesse $v$. Le produit de la force $F$ par le temps $t$ est ce qu'on appelle l'impulsion communiquée à la portion de matiere ; divisons cette impulsion par la vitesse, nous obtenons la masse maupertuisienne $m=\frac{F t}{v}$. Le produit $m v$, égal à l'impulsion $F t$, se nomme encore quantité de moutement.

La masse est donc définie comme coefficient de proportionnalité entre l'impulsion communiquée et la vitesse acquise, comme capacité d'impulsion et non plus comme coefficient d'inertie. Dans l'ancienne dynamique, il y avait identité entre les deux définitions; dans la dynamique de la relativité, la masse maupertuisienne se confond avec la masse newtonienne transversale, mais non avec la masse newtonienne longitudinale.

Nous appellerons donc dorénavant " masse "la capacité d'impulsion qui est indépendante de la direction suivant laquelle la force agit sur la portion de matière. On démontre que cette masse croît avec la vitesse $v$ suivant la loi extrêmement simple
(15)


$\left(x=1-\frac{v^{2}}{c^{2}}\right)$

mi est une constante, la masse pour $\%=1$ ou $v=0$. c’est-à-dire la masse iniliale ou masse au repos; c'est la 
valeur vers laquelle tend la masse quand la vitesse tend vers zéro.

Supposons que la vitesse d'un corps, mesurée dans un système de référence déterminé, aille constamment cn augmentant; à mesure que $c$ tend vers la vitesse $c$ de la lumière, $\%$ tend vers zéro et $m$ croit indéfiniment; la masse de toute portion de matière serait infinie si cette portion de matière était animée de la vitesse de la lumière. On voit cncore de cette manière que la vitesse de la lumière est une vitesse limite qu'on ne saurait communiquer à aucune particule matérielle, car il faudrait fournir une énergie infinic.

L'ÉNERGie ET SFs diverses ForMes. - On appelle tracail le produit d'une force par le déplacement de son point d'application dans la direction et le sens de la force, et ćnergic toute cause de production de travail cu inverecment tout résultat de la transformation d'un travail. Le travail ct l'énergie ont même mesure: dans le système C. G.S. (ccntimetre, gramme, seconde), l'unité est l'erg: c'cst le travail accomplipar une force égale à une dyne (la 981 rartie du poids du gramme) pour un déplacement de 1 centimètre dans la direction et le sens de la force.

On distingue deux catégories d'énergie : l'éner gic cinćlique et l'énergie potentielle.

L'éncrgie cinétique est l'énergie de mouvement. Uncorprsen mnuvement possède, de ce fait, de l'énergie cinétique. En mécanique classique, l'énergie cinétique d'une portion de matière (point matériel) de masse $m$ ct de vitesae $t$ est sa force vitu I

$2 m 0^{\circ}$ et l'énergie cinétique d'un systeme matéricl est la somme $\frac{1}{2} \Gamma_{m i}$ des forces vives des différents points mati- 
riels qui composent ce système. Nous verrons bientôt que cette expression ancienne de l'énergie cinélique n'est valable que comme approximation.

L'énergie potenticlle est de l'énergie en réserve, en puissance. Un exemple fera comprendre : si l'on soulève un nbjet, on dépense du travail ; ce travail n'est pas perdu, il est transformé en énergie potentielle; en effet si on lâche l'óhjet, celui-ci tombe; il y a done quelque chose qui sc transforme en énergie cinétique qui peut elle-même produire du- travail.

Dans la nature, l'énergie se présente sous des formes variées. Toul champ de force renferme de l'énergie localisée dans chaque élément de volume de l'espace.

ÉNERGIE ÉLECTROSTATIQUE. - Sil'on mel en présence un certain nombre de corps électrisés, ceux-ci excrcent des forces les uns sur les autres; le système possède de l'énergie potentielle; on modifie cette énergie en changeant les distances des charges électriques, c'est-à-dire cn dépensant (ou au contraire en récupérant) du travail. Ainsi un champ électrique, c'est-à-dire une portion d'espace où s'exercent des forces électriques possède une énergie potentielle, et l'on démontre que cette énergic est localisée dans chaque élément de volume du champ.

ENERGIE MAG.NÉTIQUE. - De même, chaque élément de volume d'un champ magnétique renferme de l'énergie, mais cette fois c'est de l'énergie cinćtiquc, car tout champ magnétique est produit par des charges (électrons) en mouvement (même un aimant renferme des charges en mouvement), et l'on sait aujourd'hui que l'énergie magnétique n’est autre chose que l'énergie cinétique de ces charges, qui est extériorisée dans l'espace environnant.

E.NERIE DU CIIAMP DE GR.AVITATION. - Les 
corps s'attirent et un système matériel possède, de ce fait, une énergie potentielle.

ÉNERGIE CHIMIQUE. - Un système de deux corps susceptibles de sunir possède de l'énergie potentielle, qui peut être transformée en chaleur et en travail lors de la combinaison de ces corps. L'énergie des explosifs est encore une forme d'énergie chimique.

ÉNERGIE C.ALORIFIQUE. - Enfin la chaleur est une des formes de l'énergie : c'est l'énergie (cinétique) du mouvement des molécules qui composent la matière.

CONSERVATION DE LEXERGIE - Un principe fondamental est celui de la conservation de l'énergie. Chaque fois que de l'énergie ou du travail se transforme, il y a production d'une énergie égale ou d'un travail équivalent. Voici deux exemples : quand on souleve un poids, l'accroissement d'énergie potentielle (énergie de gravitation) est égal au travail dépensé. Quand un corps perd sa vitesse par suite d'un frottement, toute son ćnergie cinétique se transforme en une quantité égale d'énergie calorifique, ou en une quantité égale d'énergie calorifique et d'énergie électrique (due à l'électrisation par frottement).

L'énoncé exact du principe est le suivant. L'énergie totale d'un système matériel isolé (c'est-à-dire qui n'échange aucune énergie ni aucune matiere avec l'extérieur) reste constante au cours des transformations que subit ce système.

L'INERTIE DE L'ÉNERGiE. - Unc des conséquences les plus remarquables de la théorie de la relativité est rque la notion de masse n'est pas distincle de celle d'éneigite. On démontre en effet les résuitats suivants :

I" L'énergie cinétique acquise par une particule matí- 
rielle, de masse au repos m. et de masse $m$ pour une vitesse $v$, sobtient en multipliant la variation de masse $\left(m-m_{v}\right)$ par le carré de la vitesse de la lumière. C'est seulement en première approximation (pour les vitesses faibles) que cette ćnergie est égale a $\frac{1}{2} m v^{2}$ (force vive dans la mécanique classique).

$2^{\prime}$ L'énergie rayonnante (chaleur rayonnante, lumière, ondes hertziennes) possède une masse. Une quantité d'énergie W a une masse égale au quotient de cette énergie par le carré de la vitesse de la lumière :

$$
m=\frac{\mathbb{W}}{c^{2}}
$$

$3^{\prime \prime} U_{n}$ corps qui rayonne (ou qui absorbe) de l'énergie, chaleur, lumière, etc..., éprouve une perte (ou une augmentation) de masse égale au quotient de l'énergie rayonnée (ou absorbée) par le carré de la vitesse de la lumière. En d'autres termes, en vertu du résultat qui précède, la masse de l'énergie rayonnée (ou absorbée) se trouve perdue (ou acquise) par la matière.

4" On sait que la matière est constituée par des corpuscules électrisés auxquels on a donné le nom d'électrons. M. Langevin a établi que l'énergie (potentielle) totale d'un ćlectron au repos est égale à la masse au repos de l'électron, multipliée par le carré de la vitesse de la lumière. Ce résultat sétend à toute la matière, si, comme on a toutes raisons de le penser, la matière est entièrement forméc d'électrons positifs et négatifs.

Rcunissant tous ces résultats, les conclusions suivantes simposent :

Toute verrialion d'énergic (potentielle ou cinétique) d'un 
système (formé de matière, champs électromagnétiques, rayonnements, etc.) est accompagnée d'une variation de masse de ce système, égale au quotient de la variation d'énergie par le carré de la vitesse de la lumière.

Toute forme d'énergie possède de l'inertie; la masse de la quantité d'énergie $\mathrm{W}$ est $\frac{\mathrm{W}}{\mathrm{c}^{2}}$.

Toute masse $m$ représente une énergie totale $m c^{2}$.

QUELQUES CONSÉQUENCES DE L'INERTIE DE L’ÉNERGIE (M. Langevin). - 1" VARIATION DE L I M.ASSE AVEC L. TEMPERATURE. - Une quantité d'eau dont la masse est égale à 1 gramme à la température de $0^{\prime \prime}$ doit avoir à 100" une masse plus grande. La différence (5.10 " gramme) est d'ailleurs insensible. Malgré la petitesse de cet effet, l'exemplc fait comprendre que la notion de masse cesse de se confondre avec celle de quantité de matière.

2 REACTIONS CHIMIQUES. - De la chaleur étant mise en jeu dans les réactions chimiques, comme cette chaleur a une masse, la masse du composén n'est pas rigoureusement égate à la somme des masses des composarts. Par exemple. lorsque 2 grammes d'hydrogène sunissent à 16 grammes doxygene. il se dégage sous forme de chaleur une énergie ígale at $2,87 \cdot 10^{12}$ ergs. On nobtient pas 18 grammes d'eau, mais $2,87 \cdot 10^{12}$ $9.10^{\text {t0 }}$

3" TR.WSFORMITIONS R.IDIOMCTIVFS. -- Dans Irs transformations radioactives, Penergie libére rot c.n itdérablement plus grande que dans les réactions chimiquess. Par exemple, la masse globale de l'hélium et du plomt. engendrés par tiansformation complete d'une certaine 
quantité d'uranium est certainement inférieure de plus de 10000 à la masse de cette quantité d'uranium.

Prout a émis l'hypothèse que les divers atomes sont construits à partir d'un élément primordial, l'hydrogène. Cette hypothèse de l'unité de la matière est de plus en plus confirmée par les découvertes récentes ${ }^{1}$ : par exemple, Sir Rutherford a montré que le choc d'une particule : (atome d'hélium lancé par un corps radioactif) contre un atome d'azote peut détacher de celui-ci un atome d'hydrogène. D'après la mécanique ancienne, la masse d'un atome quelconque devrait alors être un multiple exact de celle de l'atome d'hydrogène, c'est-à-dire que les poids atomiques, calculés en prenant pour unité celui de l'hydrogène, devraient étre des nombres entiers. C'est la loi de Prout, qui est effectivement à peu près vérifée car les poids atomiques sont roisins de nombres entiers; cependant il subsiste des écarts :

Lithium 6,94, bore 10,90, carbone 11,91, etc.

M. Langevin a proposé l'explication suivante : la formation des atomes (par désintégration radioactive ou par un processus inverse non encore observé, mais qui s'est nécessairement produit dans la formation des atomes lourds) a été accompagnée de variations d'énergie interne par émission ou absorption de rayonnement. La masse de l'énergie rayonnée ou absorbée est la cause des écarts ", et ceux-ci

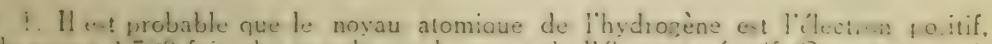

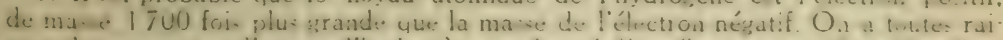


tron négatif gravitant autour du premier.

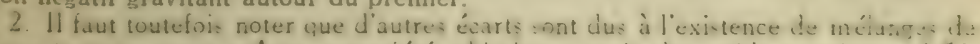

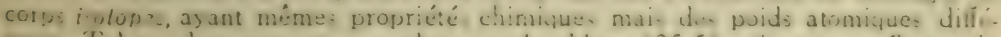
rente. Tel est le cas, par exemple, pour le chlore $(35.5$ qui e:l un mílangs do deus corps de poids atomiques 35 et 37 . 
sont tels que les énergies mises en jeu seraient du mème ordre de grandeur que celles observées au cours des transformations radioactives.

LA MATIÈRE RÉSERYOIR d’ÉNERGIE. - Soient m. la masse au repos d'un corps, $m$ sa masse pour les observateurs relativement auxquels il possède la vitesse $v$; l'énergie totale du corps, pour ces observateurs, est :

$$
\text { (16) } \begin{aligned}
W=m c^{2} & = \\
& \quad \begin{array}{l}
m c^{2} \\
1-\frac{v^{2}}{c^{2}}
\end{array} \\
& =m c^{2}+\frac{1}{2} m v^{2}+\frac{3}{8} m_{0} \frac{v^{4}}{c^{2}}+\ldots
\end{aligned}
$$

Le second terme et les suivants (en nombre infini), qui contiennent les puissances supérieures de $i$, représentent l'énergie cinétique due à la vitesse $v$ (relativement aux ohservateurs considérés). Cette énergie croit indéfiniment lorsque la vitesse $v$ tend vers la vitesse de la lumiere. Pour les faibles vitesses elle se réduit pratiquement au second tr.me $2 m v^{\prime}$ (expression ancienne de la force vive).

Le premier terme m.c. est l'énergie que renferme la matière au repos: c'est la somme des énergies cinétiques el potentielles des particules électrisées (électrons) qui. en dernière analyse, composent la matière. Cette énergie est fantastique! un seul gramme de matiere, quelle que soit li nature de celle-ci, correspond a la présence d'uné énergie interne égale à $9.10^{\circ}$ ergs, énergie qui permettrait de soulever trente millions de tonnes au somnet de la tour Eiffel, 
Presque toute cette énergie interne appartient aux noyaux atomiques, qui sont des mondes insensibles à la plupart des actions que nous pouvons produire. Une trés faible partie de l'énergie des noyaux est libérée spontanément dans les transformations radioactives. Une porticn d'énergie beaucoup plus petite encore, provenant, non plus des noyaux des atomes, mais des électrons qui gravitent autour de ces noyaux est dégagée dans le rayonnement (chaleur rayonnante, lumière, rayons $\mathrm{X}$ ) ou mise en jeu dans les réactions chimiques.

LE PRINCIPE DE LA CONSERVATION DE L.I M.ISSE SE CONFOND AVEC LE PRINCIPE DE L.I CONSERVITION DE LÉNERGIE. - Dans un systeme isolé, les diverses parties échangent de l'énergie entre elles: les masses individuelles des corps ne se conservent dor c pas; seule la masse de l'ensemble reste invariable. Le principe de la conservation de la masse n'est pas distinct du principe de la conservation de l'énergie, puisque la masse de toute substance mesure son énergie totale.

UNIFICATION DES PRINCIPES DE CONSERVATION DE I.A NASSE, DE L'ÉNERGIE ET DE LA QUANTITÉ DE: MOLVEMENT. - CONSERVATION DE L'INPUISION D'UNIVERS. - Dans la mécanique classique, en plus des deux principes de conservation de la masse et de lénersice, qui apparaissaient cemme distincts, mais cui deviennent identiques dans la dynamique nouvelle, il existe un troisieme principe : celui de la conservation de la quantité de mouvement. d'un système isolé.

Nous avons vu que la quantité de mouvement d'une parlicule de matière est le produit mo de la masse de cette

1. Sauf ceperdar.t aux rayons a (expéi iences récentes de Sir Rutherford;. 
particule par sa vitesse. C'est une quantité orientée comme la vitesse, un vecteur. Tout vecteur peut être représenté géométriquement par une portion de droite OM (fig. 11)

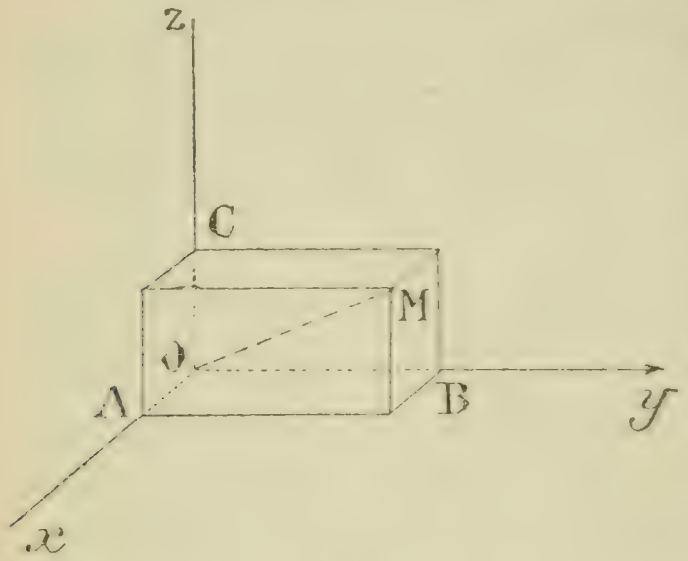

Fic. 11. ayant la direction et le sens du vecteur, et dont la longueur OM est proportionnelle à la grandeur du vecteur. On peut projeter le vecteur en $\mathrm{OA}, \mathrm{OB}, \mathrm{OC}$ sur les directions des axes de coordonnées ; l'ensemble des trois vecteurs $\mathrm{OA}, \mathrm{OB}, \mathrm{OC}$, qui sont les composantes du vecteur O.M, est entièrement équivalent à ce vecteur OM. C'est ainsi qu'on décompose les déplacements rectilignes en géométrie, les vitesses en cinématique, les forces et les quantités de mouvement en dynamique. Par la construction inverse, on peut composer en un vecteur unique OM trois rectcurs $\mathrm{OA}, \mathrm{OB}, \mathrm{OC}$ de même nature dirigés parallèlement aux axes de coordonnées.

Considérons un systène de points matériels; projeton: sur les axes de coordonnées les quantités de mouvement de tnus les points, puis ajoutons toutes les composantes suivant Ox, toutes les composantes suivant $\mathrm{O} y$, toutes les composantes suivant $\mathrm{O} z$, nous obtenons trois grandeurs $\mathrm{G}_{1}, \mathrm{G}_{1 / \text {, }}$ $\mathrm{G}$ : qui sont les composantes d'un vecteur: la quantité de: mouvement du système matériel. Nous avons ainsi composí 
en un vecteur unique l'ensemble des vecteurs quantités de mouvement de tous les points matériels du systeme.

Le principe de la conservation de la quantité de mouvement affrme que dans un système de points matériels isolé qui évolue, la quantité de mouvement de l'ensemble reste la même, c'est-à-dire que les projections $\mathrm{G}_{x}, \mathrm{G}_{y}, \mathrm{G}_{z}$ restent constantes dans un même système d'axes de coordonnées.

Lorsque, dans un même système de róférence, on change d'axes de coordonnées, les ccmposantes d'un vecteur quelconque prennent de nouvelles valeurs; il est évident que les composantes d'un vecteur se transforment suivant I mème loi qu'une distance orientée (déplacement rectiligne) puisqu'un vecteur se représente géométriquement par une portion de droite dirigée. Réciproquement, trois grandcurs physiçuement de méme nature (homogenes) qui, dans un changement d'axes de coordonnées, se transforment comme les composantes d'un déplacement rectiligne, conslituent les trois composantes d'un vecteur d'espace.

Nous allons généraliser ces notions et les étendre à l'Espace-Temps. Au lieu d'une distance, considérons un intervalle d'Univers $s$

$s^{2}=-l^{2}+c^{2} T^{2}$

$=-\left(x_{2}-x_{1}\right)^{2}-\left(y_{2}-y_{1}\right)^{2}-\left(z_{1}-z_{1}\right)^{2}+c^{2}\left(1-l_{1}\right)^{2}$

$x_{1}, y_{1}, z_{1}, t_{1} ; x_{2}, y_{2}, z_{z}, l$ étant les coordonnées d'espace ct de tomps des deux événements origine et cxtrémité de l'intervalle $s$.

De méme que la distance de deux points $\mathrm{A}$ et $\mathrm{B}$ est orientée dans l'espace, de même l'intervalle quui sépare deux événements est orienté dans l'espace-temps.

$x_{2}-x_{1}, y_{2}-y_{1}, z_{2}-z_{1}$ sont, comme en géométric, les composantes, suivant les axes de coordonnéces, de la dis- 
tance spatiale des deux événements; quant à $c\left(t_{2}-t_{1}\right)$, nous pouvons dire que c'est la composante de l'intervallc suivant le temps.

Par conséquent $x_{2}-x_{1}, y_{2}-y_{1}, z_{1}-z_{1}, c\left(t_{2}-t_{1}\right)$ sont les composantes d'espace et de temps de la portion de droite d'Univers qui sépare les deux événements. Une portion de droite d'Univers, ou ce qui est la même chose un déplacement rectiligne effectué d'un mouvement uniforme est un vecteur d'Univers à quatre dimensions, un quadrivecteur.

Par extension des propriétés des vecteurs de l'espace, lorsque quatre grandeurs physiquement de même nature se transforment, dans un changement du système de référence, comme les composantes d'une portion de droite d'Univers, c'est-à-dire (dans le cas de la relativité restreinte et avec la disposition d'axes adoptée) conformément aux formules de Lorentz, ces grandeurs constituent les composantes d'un quadrivecteur.

Un fait remarquable est que les trois composantes d'espace de la quantité de mouvement d'une portion d: matière et sa masse multipliée par la vitesse de la lumierr. (c'est-à-dire son énergie totale divisée par la vitesse de la lumière) sont quatre grandeurs jouissant de la propriété précédente. Ce sont les composantes d'un quadrivecteur, l'impulsion d'Univers.

Ce vecteur d'Univers a ainsi pour composantes d'espuat: les trois quantités de mourement suivant les dirertions des trois axes de coordonnées, et pour composante de temps l'énergic (diviséc par c) qui n’est pas orientée dans l'épare mais qui est orientée suivant le temps.

La quantité de mouyement et l'énergic (ou la masie) d'un systène matéricl apparaissent donc comme des gran- 
deurs inséparables, et les trois principes de la mécanique ancienne se réduisent maintenant à un principe unique: la conservation de l'impulsion d'Únivers.

Alors que la quantité de mouvement et l'énergie, consiĊérées séparément, ne se conservent que dans un même système de référence et changent d'un systeme de référence à l'autre, l'impulsion d'Univers a un sens absolu, indépendant du système de référence. On voit que seul l'ensemble des principes de la dynamique est absolu.

Loin de compliquer les lois de la nature, le principe de relativité, par sa puissance de simplification, conduit à unc synthese sur la beauté de laquelle il serait superflu d'insister. 


\section{VÉRIFIC.ATIONS EXPÉRIMIENT.ILES}

LFS VITFSEFS DES ÉLFCTRONS. - La thénrie de la relativité affirne que la vitesse de la lumiere ne peut pas être dépassée. Cette affirmation est la base méme de la théorie, car c'est clle qui entraine la négation du temirs absolu.

Les vitesses les plus rapides que nous connaissions sont celles des particules; (électrons) émises par les corps radioactifs. Danysz a montré que ces particules présentent toutc une série de vitesses et il est remarquable que ces vitesses convergent vers la vitesse de la lumière, allant juqu’à 297000 kil. sec. sans pouvoir atteindre 300000 kil./sec.

VERIFICATION DE I.A 1 OI D'ACCROISAFNIENT DE I A MASSE AVEC LA VITESSE. - Les expériences de M. Kaufmann et de M. Bücherer sur les rayons ; des inrps radioactifs et surtout les mesures trés précises de MM. Ch.-Eug. Guye et Lavanchy sur les rayons cathodiques (formés d'électrons animés de grandes vitesses) on prouvé que la masse de l'électron augmente avec sa vitesse. conformément à la loi prévue $m=-m_{n}$. Dans ces

$$
11-\frac{i^{2}}{c^{2}}
$$


dernières expériences la loi est vérifiée jusquà des vitesses allant jusquà la moitié de la vitesse de la lumière.

LA STRLCTURE DES RAIES SPECTRALES. - L'expérience prouve que les raies du spectre de l'hydrogène ne sont pas simples; chacune d'elles est en réalité constituée par une série de composantes extrêmement rapprochées, dont deux sont particulièrement intenses.

Un modèle d'atome, proposé par M. Bohr (un seul électron négatif tournant autour d'un électron positif dans lc cas de l'hydrogene) rend compte du spectre de l'hydroģene, mais l'application de la dynamique classique conduit à prévoir seulement des raies simples.

M. Sommerfeld a établi que la dynamique de la relativité rend compte exactement, qualitativement et quantitativcment, de la structure complexe des raies de l'hydrogene ainsi que de la structure des spectres de rayons $\mathrm{X}$. On peut considérer comme établi que la mécanique nouvelle est seule applicable aux mouvements intra-atomiques. C'est là une des plus intéressantes vérifications du principe de relativité.

LA SIGNHFICATION DE, I. ENIPERIENCE DE MICHEISON. - On présente souvent l'expérience de Michelson comme la base du principe de relativité et beaucoup de personnes oljectent quüil est scabreux de bâtir une pareille théoric sur une expérience dont le résultat a été négatif.

Il est essentiel de faire remarquer que ce n'est pas sur l'expérience de Michelson qu'il faut fonder la théorie de la relativité. Cette théorie est basée sur les formules de Lorentz, c'est-à-dire sur les lois de l'électromagnétisme car les formules de Lorentz sont implicitement contenues dans 
les équations de Maxwell : c'est le fait que ces lois ont été vérifiées par des expériences d'une extraordinaire précision et doivent être conservées quand on change de systeme de référence qui est la base inébranlable de toute la théorie. L'expérience de Michelson a joué un rôle considérable, parce qu'elle a d'abord appelé l'attention sur la discordance entre l'expérience et les prévisions déduites des lois de la mécanique; on a ensuite reconnu les causes profondes de ce désaccord. Si maintenant on donne à l'expérience de Michelson son véritable sens, on constate qu' elle vient simplement se joindre aux autres vérifications expérimentales.

La relativité généralisée et la loi de la gravitation d'Ëinstein nous apporteront des rérifications plus remarquables encore que celles qui viennent d'être indiquées. 


\section{DEUXIËME PARTIE \\ LE PRINCIPE \\ DE RELATIVITÉ GENERALISE ET LA GRAVITATION}

CHAPITRE VIII

LE CHAMP DE GRAVITATION ET L'UNIVERS RÉEL

LES SYSTĖMES GALILÉENS. - Une conception fondamentale est à la base de la théorie de la relativité restreinte : celle du mouvement rectiligne et uniforme.

Mais tout état de mouvement étant relatif, comment attribuer un sens absolu à l'état de mouvement rectiligne et uniforme? On imagine bien un mobile en translation uniforme dans un système de référence considéré, par convention, comme immobile; on conçoit que deux systemes de référence soient en mouvement rectiligne et uniforme l'un par rapport à l'autre. Existe-t-il un criterium qui permetle de décider si un système envisagé isolément est ou non en translation uniforme?

On voit qu'il est nécessaire de préciser les conditions de validité des principes et des lois précédemment exposés. Voici comment on doit résoudre la question. Nous supposons qu'on puisse trouver un système dans lequel la loi d'incrtic de Galilée (chap. 1) soil vérifiée : dans ce sy'stème, défini par un corps de référence, une particule matérielle est au repos ou se déplace d'un mouvement de: trans- 
lation uniforme par rapport au corps de référence (ou par rapport à des axes liés à ce corps) si l'on ne fait agir aucune force sur elle. Dans un tel système, appelé système galiléen, on peut adopter les coordonnées habituelles d'espace et de temps (trois axes rectangulaires pour repérer les positions et un phéncmène périodique servant d'horloge pour mesurer le temps) et ces coordonnées sont dites conrdonnées galiléennes.

Sil existe un système galiléen, il en existe une infinité d'autres : ce sont tous ceux qui sont animés par rapport au premier (et les uns par rapport aux autres) d'un mouvement de translation uniforme, sans rotation.

Ce que nous avons appelé système en translation uniforme, ou encore système non accéléré est ce que nous appeIons maintenant système galiléen. La thénrie de la relativité restreinte n'envisage que des systemes galiléens: elle affirme. que dans tout sy'stème galiléen la lumière se propage aver la même vitesse dans toutes les directions (propagation isotrope), que cette vitesse est une constante universelle, que dans chaque systeme on peut faire une mesure optique du temps (chap. III), queles lois de l'électromagnétisme (équations de Maxwell) sont rigoureuses, que les formules de transformation des coordonnées galiléennes sont celles de Lorentz, que les lois des phénomènes physiques restent lies mêmes quand on change de système galiléen.

Un espace-temps qui jouit de la propriété de contenir dans toute son étendue une infinité de systemes galiléens est un Univers de Minkowski (chap. v). Nous dirons quilil est "euclidien" à cause de l'analogie entre la ligne d'Univers qui correspond au mourement rectiligne et uniforme et la ligne droite dans l'espace de la géométrie euclidienne (p. 59) et parce que, comme l'espace de la géométrie, il 
CHAMP DE GRAVITATION ET UNIVERS RÉEL 79

est homngène, c'est-à-dire jouit des mêmes propriétés dans toute son étendue. Comme l'espace de la géométrie, cet Univers est infini.

Une question capitale se pose maintenant: l'Univers réel est-il euclidien? L'existence de la gravitation, que nous avons totalement négligée jusquà présent, ne vient-elle pas détruire l'homogénéité, qui est caractéristique de l'Univers de Minkowski?

LA PESANTELR DE L'ÉNERGIE. - Chacun sait qu'aux environs de toute matière règne un champ de gravitation, c'est-à-dire qu'en tout point de l'espace s'exerce une force, la pesanteur, qui agit sur toute portion de matière. On appelle " intensité du champ de gravitation "ou intensité de la pesanteur en un point la force qui sexerce en ce point sur une masse matérielle égale à l'unité de masse ; cette intensité dépend des masses en rironnantes (les corps sont plus légers sur la lune que sur la terre). D’après la vieille loi de Newton, deux particules matérielles de masses $m$ ct $i \prime$ 's'attireraient proportionnellement à leurs masses et ('n raison inverse du carré de leur distance $r$, de sorte que la forcc $F$ aurait pour expression

$$
\mathrm{F}=\mathrm{G} \frac{m m^{\prime}}{r^{2}}
$$

G étant une constante, la constante de la gravitation (égale a $6,7,10$ ) dans le systeme centimetre-gramne-seconde (C. G. S.).

En un point situé à la distance $r$ d'une particule unique de masse $m$, l'intensité de la pesanteur due à cette particule (force agissant sur la masse $m^{\prime}=1$ ) serait $\frac{G m}{r^{2}}$. 
La gravitation, qui agit sur toute portion de matière. agit-elle aussi sur l'énergie? la pesanteur est-elle, comme l"inertie, une propriété de l'énergie? lorsque la masse incrite d'un corps change avec son énergie interne, en est-il de même de sa masse pesante?

L'expérience répond par l'affırmative. Supposons qu'une perte d'énergie, et par suite de masse, par rayonnement, ne s'accompagne d'aucune variation de poids. Il en résulterait qu'une certaine quantité d'uranium et l'ensemble des produits de sa transformation, hélium et plomb, auraient des poids égaux mais des masses différentes. Or les expériences de M. Eotvös ont démontré (avec une précision qui atteint le vingt-millionième) qu'en tout lieu il y a proportionnalité entre la masse et le poids : la direction de la verticale, qui est celle de la résultante du poids et de la force centrifuge proportionnelle à la masse (force dinertic due à la rotation de la terre), est en effet la même pour tous les corps.

Nous sommes amenés à conclure que lénergie rayonnante, en particulier la lumière, doit etre pesante purisgi elle a une masse. Par suite un rayon lumineux doil sincurver dans un champ de gravitation.

L'ELIVALENCE ENTRE. LN CHAMIP DE: CR IVITATION IT UN CHAMP DE FORCL: DU A LX IIAT WH NOUVI:MENT ACCÉLÉE. - Les résultats qui précident entralnent de graves conséquences.

Pour un observateur lié à la terre, un mabile lancé el ahandonné à lui-méme no brét pas à la loi galileenne d"ine-1lie, puisquil est dévié par la pesanteur: nous coyons qu il en est de même pour la lumière, oe qui implique que la vitesse de la lumière ne reste pas rigoureusement constante 
CHAMP DE GRAVITATION ET L'NIVERS RÉEL 81

sur tout le parcours d'un rayon lumineux, contrairement au principe fondamental de la constance de cette vitesse.

La même conclusion s'applique partout où règne un champ de gravitation, c'est-à-dire dans l'Univers tout entier; däaucun système naturel on ne peut voir - du moins sur une grande étendue - un mobile abandonné à lui-méme ou même un rayon lumineux se propager suivant un mouvement rectiligne et uniforme ; aucun mouvement n'est conforme à la loi d'inertie de Galilée.

Mais, pensera le lecteur, ce n'est pas étonnant : la loi de Galilée s'applique, dans un système galiléen, au mobile sur leguel n'est appliquée aucune force; or dans un champ de gravitation une force attractive s'exerce sur le mobile.

Nous allons, avec Einstein, être conduits à une toute autre interprétation : si la loi d'inertie de Galilée n'est pas satisfaite, ce n'est pas parce qu'un mobile subit une force attractive sil y a un champ de gravitation, c'est parce qu'on ne peut pas trouver un système galiléen. Nous allons montrer, en effet, que la force de gravitation ne doit pas être considérée comme une force appliquée à un corps: c'est une force d'inertic absolument de même nature que celle qui apparait dans un système accéléré, c'est-à-dire dans un système non galiléen (voir fin du chap. I) ; il en résultera que dans une région où règne un champ de gravitation il n'existe pas de système de référence qui soit galiléen dans toute l"étendue du champ.

Nous allons analyser des notions qui nous paraissent évidentes parce que nous y sommes habitués, et c'est précisíment parce que nous y sommes trop habitués que personne, arant M. Einstein, n'avait eu l'idée de les approfondir.

1" L.I GRAVITATION EST UNE ICTION DE PROCHE EN PROCHE. - A la question " pourquoi un 


\section{REI_ATIVITÉ GÉNÉRALISÉE ET GRAVITATION}

objet soulevé puis abandonné à lui-même tombe-t-il ?", chacun est tenté de répondre : " parce quil est attiré par la terre ". La physique moderne doit formuler autrement la réponse.

Le développement, dans le domaine de l'électromagnétisme, de la théorie des actions de proche en proche non instantanées a conduit à la théorie de Maxwell, vérifiée par l'expérience, et au principe de relativité restreint (voir chap. IV). Une conception semblable doit être admise pour la gravitation : l'attraction de la terre sur l'objet qui tombe est un effet indirect; la propriété d'agir sur une masse matérielle ou sur un rayon lumineux appartient, en réalité, au champ de gravitation, c'est-à-dire à l'EspaceTemps qui se trouve modifié au voisinage de la matiere; ce n'est pas une action à distance, directe et instantanée, produite par un corps attirant.

2" ÉGALITÉ DE LA M.ISSE PESANTE ET DE LA MASSE INERTE. - Le champ de gravitation possede une propriété extrêmement remarquable qui n'appartient pas aux champs électrique et magnétique. Alors que dans un même champ électrique des charges différentes prennent de's accélérations différentes, dans un champ de gravtation l'accélération acquise par un corps ne dépend ni de l'état physique, ni même de la nature du corps. Tous les corps. quils soient lourds ou légers, tombent avec la méme citesse si les conditions initiales sonl les memes. L'acićlération est indépendante de la force qui sexerce sur le enrpes (indipendante de son poids).

Ce fait, si familier, est extrandinaire.

Pour les faibles vitesses, on a la loi du mouvement de Newton

lorce $=$ masse inerte $\gtrsim$ accélération 
CHAMP DE GRAVITATION ET LNIVERS RÉEL 83

c'est-à-dire que la masse inerte (masse au repos) est une constante propre au corps accéléré.

Si la force est le poids, on a

$$
\text { force }=\text { masse pesante } ~ \text { intensité du champ. }
$$

La masse pesante étant également une caractéristique du corps.

On a donc :

accélération $=\frac{\text { masse pesante }}{\text { masse inerte }} \div$ intensité du champ.

Puisque l'expérience prouve que, dans un même champ de gravitation, l'accélération est indépendante du corps, le rapport masse pesante est une constante pour tous les masse inerte

corps et si l'on choisit les unités de façon que ce rapport soit égal à 1, la masse pesante est égale à la masse inerte.

Il y a longtemps que la mécanique a enregistré ce résultat, mais personne ne l'avait interprété. L'interprétation est celle-ci : la même qualité de la matière se manifeste, selon les circonstances, soit comme inertie, soit comme pesanteur: en termes plus précis : L.I FORCE DE GR.IVI. T.ITION EST UNE FORCE DINERTIE.

Avec M. Einstein, imaginons une portion d'espace vide, si loin des étoiles et de toute matière qu'il n'y ait plus de champ de gravitation et que nous soyons dans le cas idéal nu la loi galiléenne d"inertie est applicable. Il est alors possible, dans cette portion d'Univers, de choisir un système galiléen. Dans ce système supposons une chambre isolée à lintírieur de laquelle se trouve un observateur; pour cit 
homme il n'y a pas de pesanteur, pas de direction privilégiée.

Supposons maintenant que, par un câble fixé à un crochet au milieu de la toiture de la chambre, un être extérieur se mette à tirer avec une force constante. Pour un ohservateur immobile dans le système galiléen, la chambre va prendre un nouvement uniformément accéléré et sa vitesse croitra d'une façon fantastique. Mais toute autre sera l'opinion de l'honme enfermé dans la chambre: l'accélération va projeter cet homme sur le plancher, pour lui il y aura un "haut" et un "bas" comme dans une chambre sur la terre; il constatera que tous les objets tombent avec une même accélération; sa première impression sera quil se trouve dans un champ de gravitation.

A la réflexion, il se demandera pourquoi il ne tombe pas en chute libre, ce qui ferait disparaitre la pesanteur. Cherchant ce qui se passe, il découvrira le crochet et le câble tendu; cette fois tout sera clair pour lui, il se dira : ma chambre est suspendue, au ropos, dans un champ de gravitation.

Cet homme est-il dans l'erreur? nullement : il a parfaitement le droit de considérer sa chanbre comme immohile, hien qu'elle soit accélérée relativement à l'espace galiléen. On voit que la possibilité de cette interpretation repose sur la proprićté fondamentale d'un champ de gravitation, de donner à tous les corps la même accólśration, c'est-à-dire sur l'égalité de la masse pesante et de la mass. inerte.

$3^{*}$ LE. BOULET DE JULES VERNE. - Aulieu dömaginer que la chambre de lobservateur est loin de toute matiere, supposons-he, au contraire, en chute lihre sans rotation) dans le champ de rravitation diun astre. La 
pesanteur y sera supprimée puisque tous les objets seront soumis à la même accélération que la chambre en tombant avec elle. Pour l'observateur de la chambre, il n'y aura plus ni haut ni bas, et un mobile libre sera au repos ou animí d'un mouvement rectiligne et uniforme; ce mobile se conformera à la loi de Galilée; un système de référence lié à la chambre sera donc un système galiléen (bien que pour un observateur situé sur l'astre sur lequel tombe la chambre ce système soit accéléré) et l'homme de la chambre considérera l'Univers comme euclidien dans son voisinage.

4" LE PRINCIPE D'ÉQUIV. ILENCE - Ainsi, d'une part l'emploi d'un système de référence en mouvement accéléré dans un Univers euclidien équivaut à créer un certain champ de gravitation dans lequel ce systeme pourra être considéré comme immobile; d'autre part, l'emploi d'un système de référence lié à un corps en chute libre dans un champ de gravitation revient à supprimer ce champ. En tout point d'espace il est donc impossible de se prononcer entre les deux hypothèses suivantes: $1^{\circ}$ il existe un état de mouvement accéléré sans champ de gravitation : 2" le système est au repos mais il y règne un champ de gravitation s'exerçant sur toute portion d'énergie.

En un mot il est impossible de distinguer un champ de force d'inertie dû à un état de mouvement et un champ de gravitation. Il y a ćquicalence, selon l'expression d'Einstein, qui appelle champ de gravitation tout champ de force, que ce champ soit dû à un état de mouvement du système de référence ou au voisinage de masses matérielles.

L'UNIVERS REE N'EST PAS ELCI.IDIEN. - Pour un observateur en chute libre, dans un boulet de Jules Verne, le champ de gravitation n'est supprimé que localement. 
C'est seulement dans une région peu étendue (théoriquement infiniment petite) que l'Univers est euclidien pour cet observateur. Le champ de gravitation subsiste à distance, parce que l'intensité de la pesanteur n'est constante ni en grandeur ni en direction; en supprimant le champ en un point, on l'accentue ailleurs: par exemple, relativement à un observateur qui tomberait en chute libre sur la Terre, le champ de la pesanteur serait doublé dans la région symétrique par rapport au centre de la Terre.

Dans la nature, aucun champ de gravitation n'est uniforme; aucun système de référence ne peut annuler un champ de gravitation dans toute son ćtendue. Il est impossible de trouver un système de référence dans lequel la lumière ait une propagation rigoureusement isotrope, dans lequel la loi d'inertie de Galilée puisse être rigoureusement appliquée. En un mot le système galiléen est théoriquement imaginable, et l'esprit le conçoit aisément parce que c'est le système le plus simple - de même que la géométrie euclidienne est la plus intuitive - mais ce n'est qu'une fiction et l'Unicers réel, cnvisagé dans son cnsemble, n'est pas euclidien.

LA GÉNÉRAIISATION DU PRINCIIE DE REIATTIVITÉ. - Puisque nos postulats fondamentaux ne sont pas rigoureusement vrais dans l'Univers réel, faut-il donc considérer le principe de relativité comme une abstraction en dehors des réalités? Doit-on renoncer à cette admirable synthèse et considérer l'invariance des lois de la nature comme une simple approximation? Faut-il penser que celle: invariance ne serait exacte qu' à la limite, dans un Univers euclidien et en n'envisageant que des systèmes de réfé. rence galiléens? 
CHAMP DE GRAVITATION ET UNIVERS RÉEI

Doit-on, au contraire, étendre le principe de relativité au cas de l'Univers réel et de système de référence absolument arbitraires?

M. Einstein n'a pas hésité. Il a érigé en principe l'affirmation suivante:

Tous les systèmes de référence sont équicalents pour formuler les lois de la nature: ces lois sont "covariantes" vis-à-vis de transformations de coordonnées arbitraires.

Cette généralisation s'impose. En effet toutes les lois de notre science sont basées sur la constatation de coïncidences absolues dans I'Univers. Dans le langage de la relativité, ces coïncidences sont des intersections de lignes d'Univers, absolues et par suite indépendantes de tout système de coordonnées. Il est donc certain que les lois de la nature doivent pouvoir s'exprimer sous une forme intrinsèque, une forme qui reste la même quel que soit le système de référence, quelles que soient les coordonnées choisies pour repérer les événements.

Il fallait néanmoins une certaine audace pour généraliser ainsi le principe de relativité, car les observations les plus familières semblent contredire cette généralisation. $\mathrm{Par}$ exemple: dans un véhicule, un voyageur a été jeté à terre par suite d'un coup de frein trop brusque; il parait diffcile de persuader à ce voyageur que les lois des phénomènes sont les mêmes dans un système de translation uniforme et dans un système accéléré. Voici l'explication: dans tout système de référence règne un champ de force, un champ de gravitation (au sens généralisé d'Einstein); les grandeurs caractéristiques de ce champ interviennent dans l'expression des lois; c'est lui qui se manifeste par les

1 Cela signifie oue si ces lois sont donnée: dans un sy:tème de référence, elles :ont dunnées en mêne temp dans lout autre système, quel qu'il soii. 
effets mécaniques de l'accélération. Dans le cas idéal du système galiléen, ce champ est nul: c'est précisément l'annulation du champ de force qui se traduit par la loi d'inertie de Galilée et qui caractérise le système galiléen: les lois générales doivent alors prendre, dans ce cas particulier, une forme simplifiée, disons plus exactement une forme dégénérée. Par exemple, les équations de Maxwell sont la forme dégénérée d'équations générales (auxquelles M. Einstein a pu remonter) où intervient le champ de gravitation: fait remarquable, leз lois de l'électromagnétisme sous leur forme la plus générale sont d'une extrême simplicité; elles apparaissent à l'esprit comme plus claires que les lois de Maxwell (appendice, note 14). C'est sous la forme dégénérée que ces lois ont été établies expérimentalement, parce que sur la terre le champ de gravitation (pesanteur et force centrifuge) est trop faible pour que son influence sur les phénomènes électromagnétiques ait pu être constatée. Les lois de Maxwell et les formules de Lorentz (qui sont la conséquence de ces lois) doivent étre rigoureuses dans un Univers euclidien, et si l'on prend des coordonnées galiléennes: on voit par là que la théorie de la relativité restreinte reste intacte, mais elle correspond a un cas idéal: celui où le champ de gravitation serait nul.

En résumé les équations qui expriment les lois physiques doivent pouvoir être écrites de manière à conserver la même forme dans un champ de gravitalion quelconque cest-à-dire quand on change d'une manière arbitraire le système de référence.

Cette condition de covariance limite considérablement les formes possibles pour les lois de la nature. 


\section{LES COORDONNÉES DE G.AUSS}

LE TEMPS ET LES IONGUEURS DANS LN CHAMP DE GRAVITATION. - Dans un Univers de Minkowski, imaginons un système galiléen $S$, puis prenons un second système $\mathrm{S}$ formé par un disque plan dont le mouvement, par rappcrt à $S$, est une rotation autour d'un axe normal au plan du disque, passant par le centre de ce disque, ei fixe dans le système $\mathrm{S}$. Un observateur situé excentriquement sur le disque éprouve l'effet d'une force agissant radialement vers l'extérieur; cette force est interprétée par un observateur immobile par rapport au systeme $\mathrm{S}$ comme un effet d'inertie (force centrifuge), mais l'observateur entrainé avec $S$ peut considérer son disque comme immobile et attribuer la force à un certain champ de gravitation. Ce champ possède d'ailleurs une structure fort différente de celle du champ qui s'exerce au voisinage d'une masse attirante, mais en vertu du principe d'équivalence nous l'appelons quand même champ de gravitation ou, si l'on veut, champ de gravitation géométrique.

Supposons que l'observateur de $\mathrm{S}^{\prime}$ prenne deux horloges identiques marquant toujours la même heure tant qu'on les laisse au même point; il place l'une au centre du disque et l'autre à une distance $r$ du centre; ces horloges ne vont pas rester synchrones. Examinons-les, en effet, du 
système galiléen $\mathrm{S}$, de façon à appliquer les résultats de la relativité restreinte; celle qui est au centre est immobile. l'autre est en mouvement: le temps propre de cette dernière est donc plus court que le temps du système galiléen, qui est le temps au centre. Si, au bout de quelque temps, on ramène au centre du disque l'horloge qui a séjourné à la distance $r$, on constate qu'elle retarde sur celle du centre. Comme à chaque distance au centre correspond un temps propre, iln'y a aucune synchronisation possible pour les horloges du systeme $\mathrm{S}^{\prime}$; on ne peut pas définir un temps ialabie pour le disque iout cntier, c'est-à-dire mesurable par des horloges immobiles par rapport à ce disque. La même difficulté se présente pour les coordonnées d'espace. Imaginons qu'en appliquant sur la périphérie du disque une règle très courte prise pour unité de longueur, on marque deux points $A, B$ et que le rayon soit mesuré avec la même règle unité. Pour un observateur placé au centre du disque (immobile et par conséquent appartenant au système galiléen) le rayon du disque n'est pas changé par la rotation, mais la longueur $A B$ qui est parallele à la vitesse est plus courte que si le disque ne tournait pas (contraction des longueurs); l'observateur est donc conduit à considérer la circonférence, qui contient un nombre déterminé de fois la longueur $A B$, comme plus courte, et il trouve que le rapport de la circonférence au diamère est inférieur au nombre -. La géométric de ce disque niest plus cuclidienne.

Cet exemple fait comprendre que, d'une faşon ginérale, dans un champ de gravitation géométrique (dù à un état de mouvement accéléré) on ne peut plue définir les coordonnées habituelles d'espace et de temps. En vertu du principe d'équivalence il en est de même dans un champ̧ 
de gravitation permanent (dû au voisinage de matiere) c'est-à-dire dans un univers non euclidien. Dans un univers non euclidien, il n'y a plus de coordonnées galiléennes, car la possibilité de choisir de telles coordonnées est caractéristique d'un univers euclidien.

En présence de cette difficulté, M. Einstein a résolu la question par une admirable extension de la théorie des surfaces de Gauss.

LES SURFACES ET LES COORDONNEES DE G.ILSS.Tout au début du chapitre I nous avons, pour définir ce qu'on entend par systeme de coordonnées, envisagé une surface plane. Supposons maintenant une surface courbe qui ne soit pas développable sur un plan, par exemple la surface de la terre que pour simplifier nous supposerons rigoureusement sphérique; si l'on s'interdit d'aller d'un point à l'autre de la surface en quittant celle-ci, c'est-à-dire si l'on ne considère que les points situés sur la surface même, celle-ci constitue, comme le plan, une multiplicité à deux dimensions - deux coordonnées, la longitude et ia latitude définissent la position d'un lieu sur la terre -- mais la géométrie de cette surface n'est plus la géométrie d'Euclide: il n'y a plus de lignes droites sur la sphère, et la plus courte distance d'un point à un autre est un arc de grand cercle; on ne peut plus se servir de coordonnées cartésiennes rectangulaires.

On vait que pour repérer les événements dans l'Univers réel, non euclidien, ou il n'y a plus de coordonnées rigoureusement galiléennes, nous nous trouvons, avec quatre dimensions au lieu de deux, dans la même situation que le géomètre qui veut repérer les points sur une surface courbe sans sortir de cette surface. 
Gauss a montré qu'il est possible d'énoncer les lois de la géométrie d'une surface courbe quelconque (sphère, ellipsoïde, etc.) sous une forme indépendante du système ae coordonnées. On comprend qu'en ajoutant deux dimensions on pourra, par une généralisation de cette théorie. énoncer les lois de l'Univers non euclidien à quatre dimensions.

Gauss est parti de liidée qu'il doit ètre possible, par des opérations de géodésie sur la surface, de mettre en évidence la courbure de celle-ci en faisant simplement des opérations locales d'arpentage, par les procédés habituels de la gécmétrie euclidienne du plan. En effet, en tout point d'une surface, il existe un plan tangcil et dans une étendue limitée la surface peut ètre confondue avec son plan tangent: ceci est d'autant plus exact que l'étendue envisagée autcur du point est plus petite, ei devient rigoureux. à la limite, pour une étendue infiniment petite.

Traçons sur la surface une famille de courbes artitraires $x_{1}$ (fig. 12): désignons chacune de ces courbes par un

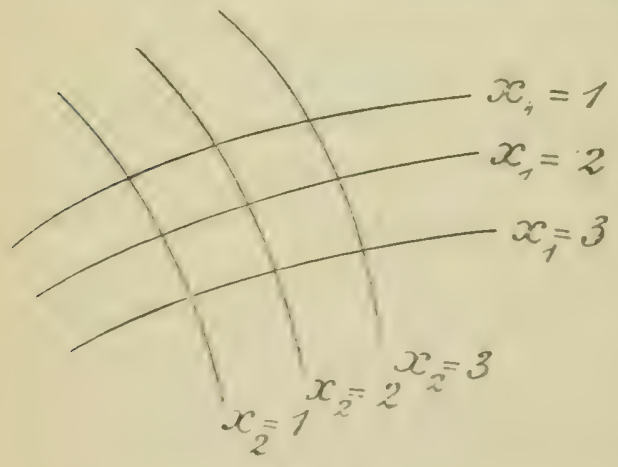

FIG. 12 chiffre et figurons les courbes $x_{1}=1$, $x_{1}=2 .$. entre deux de ces courbes, on peut imaginer une infinité de courbes représentant tousles nombres compris entre les deux nombres entiers qui désignent les deux courbes envisagées. Ces crourles sont seu!ement assujetlies à la condition de ne 
pas se couper, de façon qu'il ne passe qu'une des courbes $x_{1}$ par chaque point; de la sorte, à chaque point de la surface correspond une coordonnée $x_{1}$ bien déterminée.

Traçons de même une seconde famille de courbes $x$, les courbes $x$. coupant les courbes $x_{1}$. Chaque point de la surface est maintenant entièrement défini par les valeurs de ses deux coordonnées $x_{1}$ et $x_{2}$.

Deux points $\mathrm{P}$ et $\mathrm{P}^{\prime}$ infiniment voisins ont pour coordonnées respectives $x_{1}$ et $x_{2}, x_{1}+d x_{1}, x_{2}+d x_{2}$. Les coordonnées de Gauss reviennent, en somme, à un numérotage, à la coordination de deux nombres, faite de manière que deux points infiniment voisins soient représentés par des nombres infiniment peu différents.

Dans une étendue infiniment petite autour d'un point $\mathrm{P}$, nous confondons la surface avec son plan tangent et les courbes avec les lignes droites qui leur sont tangentes (fig. 13); nous sommes ainsi ramenés, en chaque point, à un système de coordonnées rectilignes mais obliques; une formule bien connue de la géométrie euclidienne donne la distance $d l$ du



FIc. 13 point de coordonnées $x_{1}, x_{2}$ au point infiniment voisin de coordonnées $x_{1}+d x_{1}, x_{2}+d x_{2}$

$$
d^{2}=g_{11} d x_{1}^{2}+g_{12} d x_{1} d x_{2}+g_{21} d x_{2} d x_{1}+g_{3-2} d x^{2}
$$

ou (17) dl $=g_{11} d x_{1}^{2}+2 g_{12} d x_{1} d x_{2}+g_{2} d x_{2}^{2}$

parce que $g_{21}=g_{12}$. 
Si l'on s'est donné les courbes $x_{1}$ et les courbes $x_{-}$, on peut, en chaque point $\mathrm{P}$, de coordonnées $x_{\mathrm{i}}$ et $x_{2}$, mesurer avec une règle les distances $(\vdots l)^{\prime}\left(\sum_{l}^{\prime}\right)^{\prime}(\vdots l)^{\prime \prime}$ qui séparent le point $\mathrm{P}$ de trois points extrêmement voi-

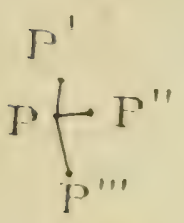

Fic. It sins de lui $\mathrm{P}^{\prime}, \mathrm{P}^{\prime}, \mathrm{P}$ (fig. 14) et correspondant à des valeurs connues des différences de coordonnées $\left(\hat{\Sigma}_{x_{1}}\right)^{\prime},\left(\hat{x}_{2}\right)^{\prime}$, etc...; toutes ces grandeurs étant extrémement petites nous pourons pratiquement les considérer comme infiniment petites, cest-à-dire écrire $(\vdots l)=(d l)^{\prime}$, etc... et appliquer, pour les trois distances, la formule (17). Nous avons danc trois équations permettant de calculer g.!, $g_{12}, g_{2: 1}$ qui sont ains! obtenus par des mesures ordinaires d'arpentage.

Conformément à la géométrie euclidienne ordinaire, les g sont bien déterminés en chaque point: ils sont indépendants des points $\mathrm{P}^{\prime}, \mathrm{P}, \mathrm{P}$ choisis pour les mesures d'arpentage. Mais, d'un point $\mathrm{P}$ à un autre. les go sont variables; ce sont des fonctions des conrdonnéce $x$ : et $x$. (c'est-à-dire des grandeurs qui dépendent des valeurs de $x_{1}$ et $\left.x_{2}\right)$. Cest seulement dans le cas d'une surfare euclidienne quion peut trcuver des lignes $x_{1}$ et $x$, telles quion ait

$$
\begin{aligned}
d l^{2} & =d x_{1}^{2}+d x_{2}^{2} \\
g_{11}=g_{22} & =1, \quad g_{12}
\end{aligned}
$$
c'est-à-dire
$g_{12}=0$

en tout point. C'est ainsi que dans le plan, on peut prendre pour $x_{1}$ et $x_{2}$ des droites rectangulaires, cest-a-dire enployer des coordonnées cartésiennes rectangulaires (chap. I. équation (1)|. L’équation (18) est coractiristique dime surface cuclidienne.

Dans le cas général, les g étant en chaque point des fonctions de $x_{1}$ et $x_{2}$, larpentage permet de calculer les 
$g$ et de déterminer comment ils varient en fonction des coordonnćes. Gauss a montré que la géométrie d'une surface est entièrement déterminée quand on connaít ces fonctions et que les lois de cette géométrie s'expriment d'une façon indépendante des coordonnées.

Il est évident que la distance $d l$ de deux points déterminés, infiniment voisins l'un de l'autre, est un invariant, c'est-à-dire a une valeur indépendante du système de coordonnées, puisque cette distance peut être assimilée à un élément de ligne droite dans le plan tangent. Considérons maintenant deux points $P_{1}$ et $P_{2}$ et une ligne courbe quelconque tracée sur la surface entre ces points: la longueur de l'arc de courbe entre $\mathrm{P}_{t}$ et $\mathrm{P}_{2}$ est (comme nous l'avons dit p. 54) l'intégrale $\int_{P_{1}}^{P_{3}} d l$; l'arc de courbe élémentaire $d l$, assimilé en chaque point de la courbe à un élément de droite dans le plan tangent en ce point, est donné par la formule (17); il a une valeur indépendante des coordonnées choisies: l'intégrale, c'est-à-dire la longueur de l'arc de courbe est, par suite, un invariant pour toute transformation de coordonnées.

Sur toute surface, il existe des lignes de plus courte distance qu'on nomme les géodésiques (sur le plan ce sont les droites, sur la surface d'une sphère ce sont les grands cercles, etc...). Si l'on exprime mathématiquement qu'une ligne jouit de la propriété d'être la plus courte entre deux quelconques de ses points, c'est-à-dire que l'intégrale $\int d l$ (où maintenant l'en ne spécifie plus les deux points $\dot{P}_{1}$ et $P$.$) est minimum, on obtient une équation qui est l'équation$ générale des géodésiques. Dans un changement du système de coordonnées, l'équation des géodésiques reste la même, à condition, bien entendu, que les g aient les nou- 


\section{RELATIVITÉ GÉNÉRALISÉE ET GRAVITATION}

velles valeurs g' correspondant aux nouvelles coordonnées $x_{1}$ et $x$. Les propriétés des géodésiques sont exprimées sous une forme indépendante du système de coordonnées; il devait bien en être ainsi car la propriété de longueur minimum qui les caractérise est absolue; elle est évidemment indépendante du fait qu'il plait au géomètre d'adopter telle ou telle décomposition de la surface en mailles à deux dimensions.

On peut aller plus loin et caractériser l’individualité de la surface en chaque point; il existe, en effet, un élément qui s'exprime au moyen des g et de ce qu'on nomme en mathématiques leurs dérivés premières et secondes. C'ct ćlement est invariant, cest-à-dire a une valeur numérique indépendante du systime de référence employé; íest ha courbure totale
(19)
$\mathrm{R}=\frac{1}{\mathrm{R}_{1} \mathrm{R}_{2}}$

$R_{1}$ et $R_{2}$ étant deux rayons de couriure quion appelle les rayons de courbure principaux.

Pour un plan, $\mathrm{R}_{1}$ et $\mathrm{R}_{2}$ sont infinis et la courbure totale est nulle en tout point. Pour un cylindre, l'un des deux rayons de couribure est infin: (à cause des génératrices rectilignes) et l'on a encore $\mathrm{R}=0$.

Si l'on suppose $\mathrm{R}$ constant et négatif on a les lois de la géométrie de Lobatchefski.

Si $R$ est constant et positif, on a la gémétric de Riemann, applicable à la surface d'une: sphère.

Extension de La THÉORIE DE Callos - Dans l'Univers réel, nous ne pouvons plus employer des coordonníes galiléennes; puisque nous ne pouvons plus défr- 
nir les coordonnées habituelles d'espace ct de temps, de mêmé qu'en géométrie des surfaces courbes, il n'y a plus de coordonnées cartésiennes rectangulaires.

En géométrie, on décompose une surface courle en mailles bidimensionnelles, avec des coordonnées $x_{1}, x$ 2 arbitraircs. De même l'Univers peut être décomposé en celluies quadridimensionnelles, avec quatre coordonnées urbitruires $x_{1}, x_{2}, x_{*}, x_{\text {. }}$. Dans le cas général, il n'y a plus ni longueurs ni temps; $x_{i}, x_{i}, x, x_{i}$ sont quatre " coordonnées d'Univers". La méthode est calquée sur celle de Gauss, avec deux dimensions de plus. Au lieu de deux familles de courbes $x_{1}$ et $x_{2}$, on a quatre familles d" " cspaces" (non cuclidiens) tridimensionnels $x_{1} x x_{2}$ : $x_{1} x_{2} x_{i}, x_{1} x_{1} x_{i}, x_{-} x_{2} x_{4}$; en chaque point d'Univers, ou événement, se coupent quatre espaces.

Il ne faudrait pas croire qu'une pareille coordination n'ait pas de sens, les coordonnées ne signiftant plus rien au point de rue des longueurs et du temps. Nous arons en effet insisté sur le fait, qui est la base même de la génèralisation du principe de relativité, que les réalités physiques correspondent aux rencontres de lignes d'Univers de portions de matière ou d'énergie. Ces rencontres s'expriment par des valeurs communes des coordonnées, quel que soit le choix de ses coordonnées; tous les systèmes sont donc également bons pour exprimer les lois de la nature, et la description de l'Univers peut se faire en coordonnées arbitraires, tout comme la géométrie des surfaces; peu importe que ces coordonnées ne soient ni des longueurs ni des temps. Le principe de relativité généralisé peut maintenant sénoncer : Tous les systèmes de Gauss (généralisés) sont équiviclents pour formuler les lois de la nature.

Tient-on cependant à conserver les notions d'espace et 
de temps? on peut le faire et l'on devra le faire dans toutes les applications physiques. Dans un systeme galiléen, on pourrait prendre un corps de référence invariable (invariable dans un méme système) par rapport auquel on repérerait les longueurs, et des horioges synchrones pour mesurer le temps; dans un champ de gravitation, ou il n'y a plus de corps invariable ni d'horloges synchrones, on envisagera comme corps de référence des corps non rigides auxquels seront liées des horloges de marche arbitraire (assujetties seulement à la condition que les indications observables d'horloges infiniment voisines diffèrent infiniment peu), ou si l'on veut un systeme formé d'un réseau arbiiraire à trois dimensions, avec des horloges aux noeuds du réseau pour donner l'heure dans chaque cellule. De pareils systemes de référence, qui non seulement sont en mouvement arbitraire mais changent de forme arbitrairemeni dans le champ de gravitation sont les " mollusques" d'Einstein. Le mollusque est un système de Gauss généralisé, mais on conserve les notions d'espace et de temps, chaque point du mollusque étant considéré comme point d'espace. chaque point matériel par rapport à lui étant considéré comme au repos, tant que ce mollusque sert de systeme de référence.

La généralisation de la théorie de Gauss peut se résumer dans le tableau suivant : 
SURFACE COURBE, NON EUCLIDIENNE

Deux dimensions. Décomposition en mailles bidimensionnelles arbitraires.

Dans une étendue infiniment petite autour de chaque point, la surface peut étre remplacée par son plan tangent.

Dans le plan tangent, la géométric euclidienne du plan est applicable, par suite :

La distance élémentaire $d l$ de deux points infiniment voisins ne dépend pas du système de coordonnéss (est un invariant).

Cette distance s'exprime par la formule :

$d i^{2}-511 d x i+g 12 d x_{1} d x=$

$$
+g_{2 i} d_{2} x_{1}+g_{23} d x
$$

avec

$$
g_{21}=g_{1}
$$

de sorte que les quatre $g$ se réduisent à trois.

La géométrie euclidienne est caractérisće par le fait qu'on peut trouver des systèmes de coordonnées dans lesquels les $g$ ont les valeurs conitantes

$$
g_{11}=g_{22}=1, \quad g_{12}=0
$$

en tuut point

'coordonnéss cartésiennes rectangulaires).

Il existe des lignes de plus courle distance (telles que $\int d l$ soit minimum) appelées géodésiques.

La courbure totale s'exprime en fonction des g et de leurs dérivées premières et secondes; cette courbure est un invariant.

\section{UNIVEIRS NON EUCLIDIE::}

Quatre dimensions. Décomposition en cellules quadridimensionnelles arbitraires.

Dans un domaine quadridimensionnel infiriment petit autour de chaque pointévénement. l'Univers peut être remplacé par son Univers euclidien tangent, qui est un Univers de Minkowski.

Cet Univers tangent est (dans une étendue suffisamment petite) l'Univers de tout observateur en chute libre, rapportant les événements à un système de référence qui lui est lié.

Dans l'Univers euclidien tangent, la relativité restreinte est applicable, par suite :

L'intervalle élémentaire $d s$ entre deux événements infiniment voisins ne dépend pas du système de coordonnézs (est un invariant).

Cèt intervalle s'exprime par la formule :

$\begin{aligned} d s^{2}= & g_{11} d x_{i}^{*}+g_{10} d x_{1} d x_{2} \\ & +g_{13} d x_{1} d x_{3}+\ldots+g_{11} d x_{1}^{2}\end{aligned}$

avec

$$
g_{\mu^{2}, \gamma_{1}}=g_{\gamma_{\gamma_{2}}}
$$

de sorte que lcs seize g se réduisent à dix.

La relativité restreinte (Univers cuclidien) est caractérisée par le fait qu'on peut trouver des systèmes de cuordonnézs dans lesquels les $g$ ont les valeurs. constantes

$$
\begin{aligned}
& g_{11}=g_{22}=g_{33}=-1, \quad g_{34}=1 \\
& g_{\mu \nu}=0, \text { si } ; \neq 0 \quad\left(i_{0}, \gamma=1,2,3,4\right)
\end{aligned}
$$

en tout événement.

(coordonnéas galiléennes).

II existe des lignes d'Univers de plus grande longueur (telles que $i d s$ soit maximum) appelées géodésiqucs.

Il existe un invariant qui s exprime en fonction des $g$ et de leurs dérivées premières el secondes. On l'appelle courbure totale d'Univers en chaque pointévénement. 
Une différence se remarque entre les propriétés geoméiriques d'une surface et celles de l'Univers. Dans le cas d'une surface, $d l^{\prime}$, qui est le carré d'une longueur, est une quantití toujours positive; dans le cas de l'Univers, $d s^{2}$ peut ètre positif ou négatif; si $d s^{2}$ est positif, l'intervalle $d$ s renrésente un tcmps multiplié par $c$; si ds est négatif, l’intervalle représente une longueur dans l'espace. Sur une surface euclidienne $d l^{2}$ est la somme de deux carrés $\left(d l^{2}=d x_{1}{ }^{2}+d x_{2}^{2}\right)$; dans l'Univers euclidien de Minkowski, $d_{s}{ }^{\prime \prime}$ s'exprime au moyen de quatre carrés $\left(d s^{2}--d x^{2}-d x_{2}^{2}-d x^{2}+d x^{2}\right.$ aves $d x=c d t)$ mais les carrés des trois composantes d'espace sont précédés du signe - alors que le carré de la composante de temps a le signe + : cette différence de signe est, suivant l'expression de M. Eddington, "Ie secret des différences que présentent les manifestations de l'espace et du temps dans la Nature".

Lorsque ds représente l'are de courbélémentaire (c'està-dire infiniment petit) d'une ligne d'Univers, $d^{2}$ est toujours positif (deux événements infiniment voisins sur une ligne d'Univers forment un couple dans le temps; voir p. 51) et ds est le temps propre élémentaire multiplié par $c$; ce n'est pas une distance spatiale. La propriété de maximum des géodésiques d'Univers (au lieu de minimum comme en géométrie) est la conséquence de ce fait.

Malgré cette différence l'analngie de prnpriéés, d'une part entre le plan et l'Univers de Minkowski, d'autre purt entre une surface courbe et l'Univers réd. est telle que nous avons le droit de dire que l'Univers de Minkowski est curlidien et rue l'Univers réel est courbe. 
CHAPITRE $X$

\section{L.I LOI DE L.I GRIVITATION (EINSTEIN)}

NATURE DE LA GRAVITATION. - Les grandeurs $g$.. $(\because, y=1,2,3,4)$ qui interviennent dans l'expression du carré de l'intervalle élémentaire et qui, dans le cas général, sont variables d'un point d'Univers à un autre, sont lesgrandeurs caractéristiques du champ de gravitation (au sens généralisé d'Einstein). Ce sont ces dix polcntiels de gravitation qui doivent figurer dans l'expression des lois physiques pour conserver à celles-ci leur forme, quel que soit le système de référence.

Dans le cas particulier d'un Univers euclidien, et si de plus les coordonnées sont galiléennes. les g... ont en tout point-événement les valeurs constantes :

$$
\begin{gathered}
g_{11}=g_{22}=g_{23}=-1, g_{4 \%}=1, \\
g_{12}=0 \text { si } \% \text { est différent de } \%
\end{gathered}
$$

et le champ de gravitation disparait; c'est le cas étudié en relativité restreinte. Les formules de transformation des coordonnées galiléennes - de celles-là seulement - sont les formules de Lorentz.

Un champ de gravitation, au sens généralisé, comporte un certain arbitraire puisqu'on peut le modifier à volonté par le choix des coordonnées, dont les ģx dépendent. Néanmoins il $y$ a une chose indépendante de tout choix de coordonnées: c'est la structure gécmétrique de l'Unicers; 
à cette structure correspond un champ de gravitation permanent, connexe de la présence ou du voisinage de la matière, auquel se superpose un champ de gravitation géométrique introduit par le choix du système de référence.

Puisque, dans le voisinage de masses matérielles, tous les corps prennent la mêrne accélération malgré leurs poids différents, il faut porter son attention, non pas sur la prétendue "force atiractive" qui est variable avec le corps, mais sur l'état de mouiement, c'esi-à-dire sur la ligne d'Unicers qui, étant la même pour tous les corps placés dans les mênes conditions initiales, doit être une caractíristique de l'Univers lui-même.

C'est bien une caractéristique de l'Univers car il est facile de démontrer que c'est une géodésique (voir tableau p. 99). Dans un Univers euclidien, et en coordonnées galiléennes, le mouvement d'un mobile abandonné à lui-même serait rectiligne et uniforme, la ligne d'Univers serait une droite d'Univers, ligne qui jouit de la propriété de longueur maximum (p. 57); toujours dans un Univers euclidien, remplaçons les coordonnées galiléennes par des coordonnées arbitraires, c'est-à-dire introduisons un champ de gravitation géométrique quelconque; il est bien évident, puisque l'élément de ligne $d s$ a une longueur indépendante du șistème de coordonnées, que la propriété de longueur maximum est conservée. Alors, le principe d'équivalence (p. 85) nous permet d'affirmer qu'il en est de mêrne dans un champ de gravitation permanent, c'est-à-dire que dans l'Univers réel, non euclidien, la ligne d'Univers d'un mobile abandonné à lui-même est encore la ligne de longueur maximum, c'est-àdire celle des géodésiques qui est déterminée par les conditions initiales du mouvement: c'est lia l'énoncé le plus général de la loi d'inertie. 
LA LOI DE LA GRAVITATIION (EINStein) 103

On voit bien maintenant quil serait faux de dire : la force de gravitation est une force attractive; un corps abandonné à lui-même n'a pas un mouvement conforme à la loi d'inertie par ce qu'il subit une force appliquée. Cette ancienne conception est inexacte car un corps abandonné à lui-même est un mobile libre et se meut toujours suivant la loi d'inertie, mais cette loi n'est plus celle de Galilée puisque dans un champ de gravitation permanent, c'est-àdire dans l'Univers non euclidien, il n'y a plus de système galiléen, plus de droites d'Univers.

La structure géométrique de l'Univers est liée à la présence de la matière et plus généralement de l'énergie. La courbure imposée par cette structure aux géodésiques, lignes d'Univers des mobiles libres, se traduit dans nos observations par un état de mouvement accéléré; le champ de gravitation est un champ de force d"inertie, mais cette force nous a donné l'illusion d'une force attractive appliquée, parce que, en fait, elle possède à nos yeux une telle apparence et que la loi de Newton, qui exprime cette prétendue force attractive, s'est trouvée être une excellente approximation dans la pratique.

LES TENSElRs. - Dans la théorie de la gravitation, l'intervalle élémentaire dont le carré (voir tableau, p. 99), qui est une grandeur indépendante du système de coordonnées $\left(x_{1}, x_{2}, x_{i}, x_{i}\right)$, s'exprime par

$$
d s^{2}=g_{11} d x_{1}^{2}+g_{12} d x_{1} d x_{2}+\ldots+g_{i} d x_{1}^{2}
$$

joue un rôle fondamental.

On est conduit, de plus, à envisager des êtres mathématiques appelés tenseurs (appendice, note 11). Un tenseur est un ensemble de grandeurs de même nature insé- 
104 RELATIVITÉ GÉNÉR.ALISÉE ET GRAVITATION

parables les unes des autres qui sont ditez " composantes du tenseur ". Le " calcul différentiel absolu ", créé par Riemann et Christoffel, développé par MM. Ricci et Levi-Civita (antérieurement à la théorie de la relativité) donne les règles (roir appendice) qui permettent de définir les tenseurs et de calculer les composantes d'un tenseur dans un nouveau système de coordonnées lorsqu' on connait ces composantes pour un premier système et que, bien entendu, la transformation de coordonnées qui relie les deux systèmes est donnée.

La propriété fondamentale des tenseurs est la suivante: quand toutes les composantes d'un tenseur sont nulles (ou sont respectivement égales aux composantes d'un autre tenseur) dans un système de coordonnées, elles sont encore toutes nulles (ou égales aux composantes de l'autre tenseur) dans tout autre système de coordonnées, arbitrairement choisi. C'est là une propriété qui donne une individualité propre à un ensemble de grandeurs possédant le caractère tensoriel.

Par suite, une loi formulée par l'annulation d'un tenseur (annulation de toutes ses composantes) ou formulice par l'égalité de deux tenscurs est indépendante de tout système de coordonnées.

Le principe de relativité exige que toutes les lois puissent être mises sous la forme tensorielle. II en résulte immédiatement que la vieille loi de la gravitation, la loi de Newton. ne peut pas être rigoureuse, car on ne peut pas la mettre sous la forme requise.

LA I.OI DE LA GRAVITATION. - La structure d'Univers, en présence d'une distribution donnće de matiere, est absolue, car elle ne saurnit être changée par le fait quil 
plait au mathématicien d'adopter tel ou tel système de coordonnées.

Par suite, lorsque les potentiels de gravitation $g_{2}$, changent avec le choix (arbitraire) du système de coordonnées, les valeurs de ces potentiels doivent rester compatibles avec une même structure d'Univers. C'est dire que les $g_{\aleph}$, sont nécessairement assujettis à certaines liaisons.

Les équations les plus générales exprimant les liaisons qui doivent exister entre les dix potentiels de gravitation pour que ceux-ci, dans un changement arbitraire de coordonnées, se modifient en restant compatibles avec une même structure d'Univers (quelle que soit d'ailleurs celle-ci) doivent être, comme toutes les lois physiques, des relations tensorielles.

Ces relations constituent la loi de la gravitation.

Pour résoudre ce problème, M. Einstein n'a eu que les données suivantes :

I" A distance infinie de toute matière (ou de tout rayonnement) l'Espace-Temps doit être euclidien.

2" La loi de conservation de l'impulsion et de l'énergie sous sa forme (tensorielle) la plus générale doit être satisfaite.

Il est remarquable que ces conditions aient suffi pour déterminer la loi.

LOI DE LA GRAVITATION DANS LE VIDE.

Le tenseur fondamental de l'espace-temps est formé par les $g . .$. ; il a pour composantes les seize $g$...

tenseur $g_{3:} \mid \begin{array}{llll}g_{11} & g_{32} & g_{1: 3} & g_{14} \\ g_{21} & g_{22} & g_{2: 3} & g_{24} \\ g_{31} & g_{32} & g_{3: 3} & g_{: 3} \\ g_{31} & g_{42} & g_{43} & g_{4 !}\end{array}$


mais ce tenseur est symétrique $\left(g_{21}=g_{1:,} g_{: 1}=g_{1: \text {, etc... })}\right.$ de sorte qu'il n'y a que dix composantes pouvant prendre des valeurs différentes.

A partir de ce tenseur fondamental, on forme un autre tenseur, appelé tenseur de Riemann-Christoffel. dont les composantes ont une expression très compliquée (voir note 11); nous désignerons ce tenseur par la notation abrégée

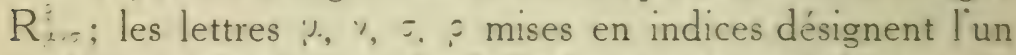
quelconque des indices $1,2,3,4$; ces mêmes indices $\%$, \%, $\tau_{,}$, figurent aussi parmi les indices des g et parmi ceux des coordonnées qui interviennent dans l'expression développée de $\mathrm{R}^{\vdots}$. Nous ne pouvons guère expliquer en langage ordinaire pourquoi $;$ est écrit en haut alors que les trois autres indices sont écrits en bas; disons seulement quil existe deux lois de transformation des composantes tensorielles lorsquion change de coordonnées: la loi de contrevariance et la loi de covariance; quand un indice est de caractère contrevariant, on l'écrit en haut, quand il est de caractere covariant on l'écrit en bas.

Comme dans le cas du tenseur g.., mais arec deux indices de plus, pour chaque valeur 1, 2, 3, 4 des indices on a une composante du tenseur, de sorte qu'en donnant successivement à toutes les lettres $:, \%, \bar{\gamma}$, i chacune des valeurs $1,2,3,+$ et faisant toutes les combinaisons possibles, on obtient 256 composantes.

On démontre que l'annulation de ce tenseur, c'est-à-dire l'annulation de toutes ses composantes est la condition nécessaire et suffisante pour que lespace-temps soit cuclidien.

(20) $\quad \mathrm{R}_{i, 5}^{\mathrm{p}}=0$.

Les 256 équations représentées symboliquement par 
cette formule se réduisent d'ailleurs à 20 équations distinctes.

Ce n'est pas la loi cherchée puisque l'Espace-Temps n'est pas euclidien dans son ensemble, mais la loi $\mathrm{R}^{\prime}=0$ convient dans une région de l'espace située à l'infini de toute masse; il faut donc chercher une relation tensorielle plus générale, comportant la précédente comme cas particulier, c'est-à-dire qui se trouve satisfaite lorsque $R_{m}^{\prime}=0$.

Partant du tenseur $\mathrm{R}_{\mathrm{v} v \pi}^{\mathrm{z}}$, à quatre indices (du quatrième ordre), on peut construire un tenseur du second ordre, c'està-dire à deux indices (seize composantes) en imposant la condition que les indices $\sigma$ et $\hat{r}$ soient toujours les mêmes (opération qu'on nomme contraction); ce nouveau tenseur, que nous désignerons par $\mathrm{R}$.. est le tenseur de RiemannChristoffel contracté ; à l'aide de ce tenseur contracté, on peut enfin former un tenseur d'ordre nul (une seule composante): or tout tenseur d'ordre nul est un invariant. L'invariant en question, $R$, se trouve être une généralisation de la courbure de Gauss (p. 96), on peut donc l'appeler courbure totale d'Univers.

La loi $\mathrm{R}=0$, c'est-à-dire l'annulation de la courbure totale en tout point-événement ne saurait convenir non plus, car c'est une loi trop générale, insuffisante pour déterminer un champ de gravitation.

On n'a donc pas le choix, car pour que $\mathrm{R}^{\prime}=0$ soit une solution particulière, il n'y a qu'une loi générale possible, l'annulation du tenseur contracté
(21)
$\mathrm{R} .=0$.

C'est la loi générale de la gravitation dans le vide (appendice, note 12).

Le tenseur $\mathrm{R}_{\ldots}$, étant symétrique $\left(\mathrm{R} .=\mathrm{R}_{. .}\right)$n'a que 
dix composantes distinctes; l'annulation des composantes donne donc dix équations. Mais on constate que six seulement de ces équations sont indépendantes: c'était à prévoir par :e que dix équations indépendantes détermineraient les dix g. dans l'expression du carré de l'intervalle $d s^{2}$, et par conséquent spécifieraient non seulement la structure de l'Espace-Temps mais encore le système de coordonnées. Or ce système doit rester arbitraire; il est quatre fois indéterminé puisqu'il y a quatre coordonnées: il faut donc qu il y ait entre les g.. quatre relations qui soient des identités (appendice. note 12).

En définitive la loi de la gravitation dans le vide comporte six conditions. C'est une restriction considérable imposée aux géométries de l'Univers.

LOI DE LA GRAVITATION D.ANS LA MATIËRE. Les équations résumées par $R=0$ décrivent les proprictés les plus générales de la structure géométrique de l'Univers aux points où il n'y a ni matière ni énergie électromagnétique. c'est-à-dire dans ce que nous appelons le vide. Il reste à résoudre un problème fondamental : la matière subit l'action d'un champ de gravitation mais nous savons qu'elle est ausai la source d'un champ de gravitation: c'est ce qu'il s'agit d'exprimer. En d'autres termes, il s'agit de déterminer la loi qui doit remplacer la loi d'attraction proportionnelle à la masse et inversement proportionnelle au carré de la distance, la vieille loi de Newton que Poisson a traduite analytiquement par une équation locale. Cest-i-dire. par une équation valable en chaque point d'espace: dans cette équation intervient la densilé de la matiere au proint considéré.

La densité en un point est le rapport de la masse contenue. dans un volume d'espace infiniment petit, à ce volume lui- 
méme: ćest la masse par unité de volume. Or la masae, c'est l'énergie diviséc par la constante $c^{2}$, et nous avons vu (p. 72) que l'énergie est inséparable de la quantité de mouvement. Mais l'énergie et la quantité de mouvement ne suffisent pas, dans le cas général, pour constituer un tenseur: 1) faut y joindre des grandeurs qui expriment les courants de matière, en d'autres termes, les courants de quantité de de mouvement. On forme ainsi un tenseur, le tenseur matériel ou ienscur impulsion-énergie que nous désignerons par T... (seize composantes) dont dix seu'ement sont distinctes car il est symétrique. C'est ce tenseur qui doit remplacer la densité qui figurait seule dans l'ancienne théorie: signalons d'ailleurs que la composante $T_{i ;}$ de ce tenseur est, dans tous les cas nù la matière est animée de vitesses faibles par rapport à la vitesse de la lumière, considérablement plus grande que les autres composantes, et serait précisément égale à la densité de la matière, si l'on pouvait employer des coordonnées rigoureusement galiléennes.

On démontre (voir une démonstration intuitive dans l'appendice, note 12) que, pour que la loi générale de conservation de l'impulsion-énergie et que la loi de la gravitation dans le vide $R_{\ldots}=0$ soient satisfaites, il doit y avoir, à un facteur constant près, égalité entre le tenseur $T_{\ldots}$, et le tenseur $R .-\frac{1}{2}$ s... $R(R$ est l'invariant dont il a été question plus haut, la courbure totale de l'espace-temps). On doit donc avoir en tout point

$$
\text { (22) } \quad \mathrm{R}-\frac{1}{2} g_{1} \mathrm{R}=-\% \mathrm{~T}_{\cdots}
$$

$\%$ étant une constante universelle. 
110 RELATIVITÉ GÉNÉRALISÉE ET GR.AWITATION

Cette loi peut encore se mettre sous la forme suivante
(23)

$$
R_{k, \nu}=-\%\left(T_{i,}-\frac{1}{2} g_{i,} T\right)
$$

T étant un incariant qu'on constiuit à partir de $T$... et qu'on trouve égal à \%, po étant la densitć au repos de la matière au point considéré. c’est-à-dire la densité qui serait mesurée par un observateur immobile par rapport à la matière ".

Dans le vide $T$,.. et $T$ sont nuls, puisqu'il n'y a pas de matière et l'on retrouve bien la loi dans le vide $R_{1}=0$.

LOI DE NEWTON. - Si, dans les dix équations représentées par (22) ou (23), obtenues en donnant aux indices $y$ et " les valeurs 1, 2, 3, 4 (les seize équations se réduisent à dix parce que les tenseurs sont symétriques: $R_{1}=R_{\text {. et }}$ $\mathrm{T}_{\mathrm{F}}=\mathrm{T}_{\text {. }}$ ) on néglige les quantités qui pratiquement sont très petites, on trouve, en premiere approximation mais non comme loi exacte, la loi de Newton, et la constante \% se trouve déterminée en fonction de la constante connue $\mathrm{G}$ qui intervient dans l'expression de l'ancienne loi.

LA DYN.IMIQLE. - Nous avons dit que, dans le vide, les dix équations $R=0$ se réduisent à six conditions, à cause de quatre identités qui correspondent à la quadruple indétermination des coordonnées.

En tout point où il y a de la matière présente, les quatre mêmes identités sont encore vérifiées, car elles résultent de: la définition mathématique du tenseur R,. Comme, d'autre part, la loi de la gravitation exprimée par (22) ou (23) traduit une relation entre $R$.. et $T$... les quatre ilintitis se transforment en quatre ćquation.s - entre les grandeurs qui

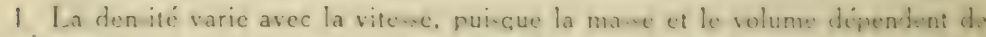
la vilesse.

2. Les cquation sont des relation: entre de zrandeur inconnue et des gran-
} 
forment le tenseur impulsion-énergie. Le degré d’indétermination des coordonnées, c'est-à-dire le nombre de dimensions de l'Univers impose donc à la matière un nombre égal de conditions qui doivent être nécessairement remplies. II se trouve que ces quatre conditions constituent la los fondamentale de conservation de la quantité de mouvement et de l'énergie (voir appendice, note 12).

Si l'on demandait "la loi d'Einstein est-elle bien d'accord avec les lois de la mécanique" il faudrait répondre: c'esl elle qui résume la dynamique tout entière. Cette loi contient. sous sa forme la plus générale, la loi de conservation de la quantité de mouvement, de l'énergie et de la masse; on démontre (voir note 12) qu'elle contient la loi du mouvement du mobile libre (la ligne d'Univers du mobile libre est une géodésique): c'est la loi de l"inertie car gravitation et inertie sont une seule et même chose. Eile contient toutc la dynamique du point matériel.

deur: connues: $c$ s sont des conditions impo:ées aux srandeurs inconnues, ces condition., si elles sont suffi:antes, déterminent les inconnuts. Dans uns idcntitc, le- deux membres expriment une seule et méme cho:e et peuvent se ramener ḋ une même exprassion; autrement dit les termes d'une identité se détruisent deux à deux. 


\section{IPPLIC. ITIO \S ET VERIFIC. ITIONS \\ DE LA LOI D'EINSTEIN}

LE CHANP DE GR AITATION D L N CHNTRE MATHRIEI. - La loi d'Einstein a permis de déterminer l'expression de l'intervalle élémentaire $d s$ qui sépare deux événements intiniment voisins dans le champ de gravitation produit par un centre matériel, c'est-à-dire l'cxpression de l'élément de temps propre ( $\left.d==\frac{d s}{c}\right)$. Celte expression (note 13) a etédnnnie en fonction de coordonnées aussi voisines que ponsithle. de coordonnées polaires cuclidiennes (roir p. 12), et qu'on peut assimiler pratiquement à des coordonnes euclidicuncs, tant que la déformation (par rapport à un espace-l(muls euclidien) de l'Univers est faible; elle s'applique dans un système de référence lié au centre source du champ de gravitation ".

Dans un champ non statique, c'est-à-dire si l'on suppos: que le centre matériel est en mouvement dins le systeme de référence employé, on trouve que les petites déformations de l'Espace-Temps, c'est-à-dire les cffels de gravilation, se propagent acee la vitesse de la lumicre. Voila résolu un problème qui avait pendant bien longtemps préoccupé les physiciens et les astronomes.

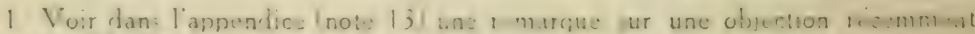
faite par M. Painlevé. 
LE MOUVEMENT DES PLINETES - PRENIERE VERIFIC ITION: LE MOUVEMENT DE MERCURE - Si I'on détermine les géodésiques de l'Espace-Temps ayant l'élément d'arc calculé ainsi qu'il a été dit plus haut, on otient le mouvement des mobiles libres dans le champ de gravitation d'un centre. Au lieu d'une ellipse fixe pour la trajectoire des planètes (mouvement conforme à la loi de Newton) on trouve une ellipse qui tourne lentement dans son plan (appendice, note 13). Le calcul numérique montre que pour les planètes autres que Mercure, l'écart entre les prévisions conformes à la loi de Newton et celles qui résultent de la loi d'Einstein est très faible, de l'ordre de grandeur des erreurs d'observation.

Mais pour Mercure, dont l'orbite a une forte excentricité et qui cst près du Soleil, si l'on introduit dans la formule les valeurs connues de la masse du Soleil, du grand axe et de l'excentricité de l'orbite, et de la durée de révolution de Mercure on trouve une rotation de périhélie (point le l'orbite le plus rapproché du soleil) de 42,9 secondes d'arc par siècle.

Depuis que Leverrier a ćtabli la théorie de Mercure, en tenant compte des perturbations dues aux autres planètes, de Vénus en particulier, le désaccord entre les prévisions de la mécanique newtonienne et les observations est précisément 43 par siècle, ćcart qu'on n'avait pas réussi à expliquer.

La nouvelle mécanique céleste basée sur la loi d'Einstein se développe actuellement, en particulier en ce qui concerne la théorie de la Lune.

SECONDE VERIFIC ITION - DEVIITION DE LA LUMIERE. - La ligne d'Univers d'un rayon lumineux est une géodésique de longueur nulle, puisque $d s$ est constam- 
ment nul pour la lumière (p. 57). La trajectoire d'un rayon lumineux s'obtient en écrivant cette condition.

On trouve (note 13) que si un rayon lumineux se dirige sur le centre matériel (propagation radiale) la vitesse de la lumière diminue à mesure que

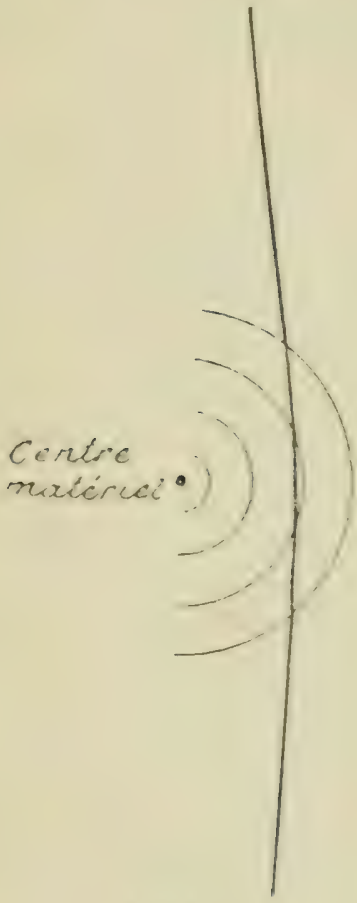

FIG. 15 la lumière se rapproche de ce centre, et le même résultat serait exact pour un mobile animé d'une vitesse très voisine de la vitesse de la lumière: c'est une véritable répulsion. Pour un rayon passant transversalement, la vitesse en un point de ce rayon est d'autant plus faible que la distance de ce point au centre matériel est moindre; il en résulte que tout se passe comme si le rayon lumincux traversait, dans un espace euclidien, un milieu réfringent réparti en couches concentriques dont lindice de réfraction augmenterait à mesure que la distance au centre serait plus petite; il est facile de voir que la trajectoire sincurve en donnant toutes les apparences d'une altraction de la lumiere par le centre (fig. 15).

Ces résultats montrent quil est, au fond, inexat d. qualifier le centre matériel de "masse attirante". La matière est un centre de déformation de l'Espace-Temps et l'effet produit sur un mobile nous apparait, selon la grandeur el l'orientation de la vitesse. soit sous l'aspect d'une 
attraction, soit sous l'aspect d'une répulsion. Nous sommes loin des idées newtoniennes.

Il est essentiel de remarquer que les résultats qui précèdent sont établis dans un système de référence lié au centre matériel et en prenant comme coordonnées des coordonnées aussi voisines que possible des coordonnées euclidiennes (coordonnées devenant d'ailleurs euclidiennes à distance infinie du centre). Il n'en reste pas moins vrai que l'observateur en chute libre (c'est le cas de l'observateur terrestre dans le champ de gravitation du Soleil, car la Terre est en chute libre), faisant avec des règles et des horloges, dans son voisinage immédiat, la mesure de la vitesse de la lumière, confondrait l'Univers réel avec l'Univers euclidien tangent et trouverait toujours dans toutes les directions une vitesse égale à la constante $c$; la variation de vitesse ne peut apparaître que si l'on envisage une très grande étendue du champ de gravitation.

Le calcul montre que pour un rayon venant de très loin, et parvenu très bin du centre après être passé à la distance minimum $\mathrm{R}$ de ce centre, la déviation totale de ce rayon cst $\frac{4 \mathrm{GM}}{c^{2} \mathrm{R}}, \mathrm{M}$ étant la masse du centre, et $\mathrm{G}$ la constante de la gravitation. Celte déviation est cxactement disuble de celle qui résulterait de la loi de Neu'on, appliquée à un mobile de vitesse initiale c. Pour un rayon passant tangentiellement au bord du soleil, on trouve 1",74: ainsi une étoile, vue près du bord du Solcil, doit être dévićc vers l'extérieur du Soleil, de 1",74 à partir de sa position normale sur la sphère céleste.

Malgré la petitesse de l'effet, l'exactitude de ce résultat a été vérifiée par les astronomes de Grcenwich et d'Oxfrrd, au cours de l"éclipse totale de Soleil du 29 mai 1919. La 


\section{II6 RELATIVITÉ GÉNÉR.ALISÉE ET GRAVITATION}

zone de totalité traversait l'Atlantique, commençant au Brésil ct finissant en Afrique. Une première expédition (MM. Crommelin et Davidson) se rendit à Sobral au Présil, et prit une dizaine de photographies pendant les cinq minutes que dura la totalité de l'éclipse. Deux mois après, la même région du ciel fut visible de nuit et fut photographiee avec les mêmes appareils pour permettre la comparaison; on trouva que les déplacements des étoiles sont bien, comme le préroit la théorie, en raison inverse de la distance au centre du Soleil, et les déplacements ramenés à ce qu'ils seraient au bord même du Soleil, ont donné une moyenne de 1",98. L’autre expédition (MM. Eddington et Cottingham), installée dans l'ỉle du Prince (golfe de Cuinée), a trouvé une moyenne de 1",60. La moyenne des deux résultats, 1,79, concorde remarquablement avec la valeur prévue par Einstein.

La déviation observée ne peut d'ailleurs pas être attribuée à une atmosphère ou à de la matière cosmique entourant le Soleil jusqua aux distances pour lesquelles les mesures ont été faites, car le pouvoir absorbant et la densité d’une telle atmosphère auraient affaibli notablement l'éclat des étsiles; d'autre part, des comètes suivies dans la même région n'ont manifesté aucun ralentissement.

TROISIE.ME VÉRIFIC. ITION- LE DÉPL. ICE.MENT DES R.MIES SPECTR.\LES. - La formule qui donne l'expression de $d s^{2}$ dans un champ de gravitation permet d'établir que, si deux horloges identiques sont placées, l'une sur la Terre ou le champ de gravitation est faible, l'autre sur le Soleil ou le champ est intense, pour l'observateur situé sur la Terre l'horloge solaire a une marche plus lente que l'horloge terrestre (note 13).

On démontre que ce résultat entraine la conséquence 
suivante. Considérons une des vapeurs lumineuses présentes sur le Soleil; les raies spectrales de cette vapeur doivent, dans le spectre solaire, nous paraitre déplacées vers le rouge, par rapport à la position des raies de la même vapeur dans le spectre obtenu au laboratoire. Ce déplacement est très faible $(0,011$ unité d'Angström pour le milieu du spectre).

M. A. Pérot, aỹant comparé dans le spectre solaire et dans le spectre obtenu au laboratoire les positions de raies du cyanogène et du magnésium, a établi qu'après corrections de déplacements dus à des causes connues, il subsiste un écart égal, dans les limites d'approximation des mesures, à celui prévu par Einstein. Le même résultat a été obtenu par MM. Buisson et Ch. Fabry pour de nombreuses raies du fer. 


\section{LA COURBURE DE L'ESPACE ET DU TEMIPS. - HYPOTHESES COSMOLOGIQUES}

L'ESPACE FINI BIEN QL'ILIAMIITÉ. - L'ancienne conception de l'espace infini comporte des contradiclions connues depuis longtemps. Doit-on admettre que dans cet espace infini la matière est répandue partout arec une densité movenne constante (l'unité de volume étant prisc. suffisamment grande) ? Ce serait admettre une quantité infinie de matière: on peut démontrer que dans cette hypothèse la loi d'Einstein, comme celle de Newton, conduirait à des résultats contradictoires.

Doit-on admettre que l'Univers a une sorte de centre près duquel la densité de la matière est maximum et autour duquel la matière se raréfie jusqu'au vide complet? La matière formerait une ile dans l'espace infini. Mais alors tonte inergie rayonnante sortie de cette ile se propageruit à l'infıni, sans retour, et se dissiperait; la matière elle-méme se disperserait, comme l'atmosphère d'un astre qui s'évapore peu à peu dans l'espace. Il faudrait admettre que, puisque l'Univers n'est pas mort, la matiere nexiste gue depuis un tumps limiti, ce qui recule toutes les difficultés et n'cn résout aucune.

Pour un homme intelligent quion aurait laiss: d.uns lignorane de la forme de la Terre. la disparition foro- 


\section{LA COURBURE DE L'ESPACE ET DL TEMPS 119}

gressive d'un navire sous l'horizon serait une révélation: ayant compris que la surface est courbe, cet homme envisagerait la possibilité d'une surface finie, d'un monde fermé. Pareille révélation est donnée par la théorie d'Einstein, par le simple fait qu'un rayon lumineux ne se propage pas nécessairement en ligne droite dans le vide, par la notion de courbure de l'Univers. On supprimerait les difficultés de l'ancienne conception en admettant que l'espace est fini bien qu'illimité, comme la surface d'une sphère qui ne comporte pas de bornes puisqu'on peut en faire le tour indéfiniment. Le temps seul resterait infini.

Ce n'est pas là une hypothèse arbitraire. M. Einstein a établi, par des considérations basées sur la théorie de l'électromagnétisme (voir cette théorie dans l'appendice, note 14), sur les propriétés du tenseur d'énergie électromagnétique (quion doit ajouter au tenseur d'impulsion-énergie matérielle quand il $\mathrm{y}$ a un champ électromagnétique) sur la théorie électronique de la matière, que la loi de gravitation quili avait primitivement donnée - celle que nous avons admise jusquà présent - doit être corrigée (note 15).

R. étant, comme précédemment, le tenseur de RiemannChristoffel contracté (p. 107), posons $\mathrm{R}^{\prime}=\mathrm{R}_{\mathrm{*},}-\lambda \mathrm{g}_{\mu,}, \lambda$ étant une constante universelle, d'ailleurs extrêmement petite. La loi dans le vide doit être
(24)
$\mathrm{R}^{\prime}=0$
au lieu de
$\mathrm{R}_{3,}=0$

et la loi, en tout point où se trouve de la matière ou de l'énergic électromagnétique sexprime par

$$
\mathrm{R}_{*}^{\prime}-\frac{1}{2} g_{*} \mathrm{R}^{\prime}=-\% \mathrm{~T}_{*}
$$

au lieu de $R_{w}-\frac{1}{2} g \ldots, R=-\% T_{w}$ 
$\mathrm{R}$ ' étant l'invariant $\mathrm{R}-4$ \%, et $\mathrm{T}$.. le tenseur total d'énergie (tenseur matériel + tenseur d'énergie électromagnétique).

Une modification radicale est la conséquence de la nouvelle loi. Alors que dans la loi primitive la courbure totale $\mathrm{R}$

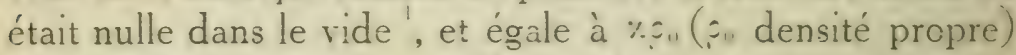
dans la matière, maintenant la courbure dans le vide est la constante $\mathrm{R}_{i}=4 \%$ et la courbure dans la matière est $\mathrm{R}=\mathrm{R}_{0}+\% \mathrm{o}_{0}$

Mais, à part ce qui vient d'être dit, rien n'est changé à la théorie, où il suffit de remplacer $R_{\ldots}$. par $R$. et $R$ par $\mathrm{R}^{\prime}=\mathrm{R}-4 \%$. La nouvelle loi entraine, comme la précédente. la conservation de l'impulsion et de l'énergie. D'ailleurs le terme correctif i.g. étant très petit, on peut le supposer nul dans toutes les applications astronomiques.

Les vitesses relatives des astres sont toujours extrêmement petites par rapport à la vitesse de la lumière. Cette remarque nous permet d'envisager un systeme de référence relativement auquel la matière est en moyenne au repos et dans lequel les vitesses individuelles sont faibles. Dans ce système la matière est quasi-stationnaire. Adoptant ce système, si l'on cherche l'aspect d'ensemble de l'Univers, en négligeant les perturbations locales dues à la distribution irrégulière de la matière (comparables au relief du sol par rapport à la forme d'ensemble de la terre), la nouvelle loi comporte deux solutions données, l'une par M. Einstein, l'autre par M. de Sitter (note 15). Dans l'une comme dans l'autre la "coupe à temps constant" est un espace à crutrbure constante positive. L'espace est fermé.

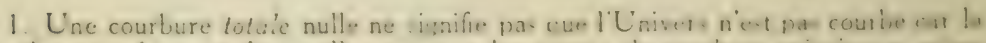

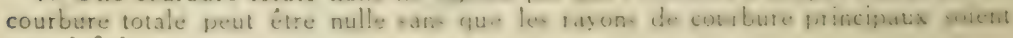
tous infinis. 
L'univers d'Einstein. - Pour mieux comprendre, considérons d'abord une surface courbe au lieu d'un espace courbe. Imaginons des êtres infiniment plats, entourés d'objets tout en surface, assujettis à vivre sur la surface d'une sphère (fig. 15) sans avoir la perception d'une troisième dimension d'espace. Confondant en chaque point $\mathrm{P}$ la surface de leur monde sphérique avec le plan tangent PT, ils imagineront la géométrie plane (celle d'Euclide) et penseront d'abord que leur univers s'étend à l'infini. Ils appelleront "ligne droite" le plus court chemin d'un point à un autre. S'ils portent autour d'un même point $P$, dans toutes les directions, des longueurs égales, ils construiront un cercle, et tant que le rayon sera petit, ils trouveront que le rapport de la circonférence au diamètre est un



Fic. 16. nombre indépendant du rayon $\div=3,1415 \ldots$ Cependant, s'ils tracent des cercles de "rayons" de plus en plus grands $\mathrm{PP}_{1}, \mathrm{PP}_{2}$ etc. - ce qu'ils appellent rayon étant un arc de grand cercle puisqu'ils restent sur la surface - ils constateront que le rapport de la circonférence au diamètre devient inférieur à - et diminue à mesure que le rayon augmente, enfin que la circonférence elle-même décroît et finit par se réduire à un point $\mathrm{P}^{\prime}$ - le point antipode. Les mathématiciens de ce 
monde comprendront que leur univers est courbe ; ils déduiront de leurs mesures d'arpentage que c'est une surface à courbure constante positive finie bien qu'illimitée, limitant un "hypercercle" à trois dimensions dont ils pourront calculer le rayon.

Ajoutons une dimension, nous pourons conceroir l'cspace sphérique. Cet espace est difficile à se représenter ; il n'a absolument rien de commun avec l'intérieur d'une boule limitée par une surface sphérique dans l'espace à trois dimensions: il limite une hypersphère dans un espace à quatre dimensions comme une surface sphérique limite une sphere ordinaire. Dans l'espace courbe qui limite une hypersphère. portons à partir d'un point, dans toutes les directions, de's longueurs égales mesurées sur des fils tendus, nous obtenons une sphère. A partir du même point portons des longueurs de plus en plus grandes, nous obtenons d'abord des sphères de surfaces croissantes, puis une sphere maximum (pour la longueur $\frac{1}{2} \approx r, r$ étant le rayon de l'hypersphère) ensuite les sphères décroissent - comme les cercles de l'exemple précédent - pour se réduire au point antipode à la distance $\pi r$.

Dans l'Univers d'Einstein, l'espace est sphérique' mais le: templs na pas de courlure, il est rectiligne: l'Fspace- T cmps cst culindrique. Cette hypothèse constitue un retour it l'espace absolu et au temps ahsolu; la séparation entre lispace et le temps cost ritablie, paree que la direction des génératrices du cylindre donne un temps d Uniters atowh. Mais c'est un ahsolu dont nous navons pas connaisane en toule rigueur, iar, pour tout olservateur en mouve-

1. Ou elliptique, mais nous ne parlerons que de l'espace sphérique. 
LA COURBURE DE L'ESPACE ET DL TEMPS 123

ment par rapport à l'ensemble de la matière mondiale, l'espace et le temps restent unis suivant la conception de Minkowski; le temps que nous mesurons, variable d'un système à l'autre, variable d'un point à un autre dans un champ de gravitation, n'est pas ce temps absolu; toutefois l'écart est bien faible, il ne serait notable que si l'on parvenait à réaliser des vitesses considérables relativement à l'ensemble de la matière mondiale.

Une conséquence curieuse est que les rayons lumineux émanés d'un point, après s'être concentrés au point antipode, pourraient se concentrer de nouveau au point de départ, qui ne serait plus d'ailleurs le point occupé par la source de lumière car celle-ci se serait déplacée pendant le temps - des billions ou des trillions d'années peut-être - que demanderait la lumière à faire le tour de l'Univers. Beaucoup d'étoiles ne seraient que des fantômes d'un passé très reculé. Mais cette conception est peu rraisemblable; il est bien probable que la lumière serait absorbée dans un pareil voyage, car il $y$ a toujours des traces de matière répandues dans l'espace.

L'Univers de DE SitTer. - Dans la solution de M. de Sitter la coupe à temps constant est encore un espace sphérique, mais il y a aussi une courbure du temps. L'Univers est hyperbolique. Il n'y a plus de temps d'Univers al solu; l'espace et le temps restent unis: c'est la relativité dans toute sa plénitude.

Une conséquence remarquable de la courbure du templs est que le temps qui sécoule entre deux événements sc: produisant, relativement à l'olsservateur, en un même point d'esprace, parait à cet olservateur d'autant plus long que le. point est plus voisin d'une certaine zone où le lempes est 
stationnaire (l'observateur ne perçoit pas le temps propre de cette zone, parce que ce temps et le sien sont orthogonaux) (note 15).

Mais ceci n'est qu'un point de vue relatif à l'observateur et ne signifie pas que le cours du temps soit arrêté dans cette zone; si l'observateur s'y transportait, it trouverait que la Nature y est aussi actire que partout ailleurs, et c'est son ancienne demeure qui lui paraitrait immobilisée dans un repos éternel.

La lumière elle-même demanderait un temps infini pour parvenir à la zone du temps stationnaire; alors, plus de fantômes d'étoiles, car il y a la barrière du temps; pour l'observateur, jamais un mobile, jamais un rayon de lumière ne franchiront cette barrière. Et pourtant, si l'observateur pouvait mesurer la vitesse d'un mobile à mesure qu'il s'éloigne, il trouverait que cette vitesse (et à fortiori celle de la lumière) croît indéfiniment! Ce serait, pour l'homme auquel il manque une dimension pour percevoir directement la courbure, l'illusion complete d'un Univers infini dans l'espace comme il est infini dans le temps.

On se demande si les déplacements des raies spectrales des nébuleuses spirales (mondes extrêmement lointains), déplacements qui ont presque toujours lieu vers le rouge, ne seraient pas la manifestation du ralentissement apparent du temps, c'est-à-dire de la courbure du temps qui pourrait se manifester sur de si grandes distances.

L'ACCÉlÉRATION ET LA ROTATION. - Nous avons déjà insisté sur le fait que toute accélération semble posséder un caractère absolu. L'explication est la suivante: les lignes d'Univers naturelles, ou gćodésiques, ont une signi- 
fication absolue: elles sont déterminées par la structure séométrique de l'Espace-Temys. En tout point-événement, il existe un Univers tangent, l'Univers euclidien de l'observateur en chute libre; dans un système de référence lić à cet observateur, ou dans un systeme en trans'ation uniforme par rapport à lui, les gúodésiques peuvent être à très peu près confondues avec des droites d'Univers dans une grande étendue. Le mouvement de translation uniforme n'a aucun caractère absolu puisquil conserve aux géodésiques leur forme rectiligne: il ne peut pas être déterminé par rapport aux géodésiques. Au contraire toute accélération (et en particulier toute rotation) par rapport à ces lignes d'Univers a une réalité objective. C'est cette réalité qui est observée avec le pendule de Foucault qui permet de constater la rotation de la terre.

LA STRUCTURE D'UNIVERS ET L'ÉTHER. - L'Univers possède une structure géométrique connexe de la présence de matière ou d'énergie électromagnétique, puisque le champ de gravitation qui règne au voisinage de la matière (ou de l'énergie) n'est autre chose qu'une déformation de l'EspaceTemps.

Si l'on cherche à préciser la relation qui doit exister entre la structure de l'Espace-Temps et la matière, deux points de vue opposés peuvent être envisagés.

1" On peut attribuer à la matière, ou plus exactement aux électrons qui la composent, un rôle primordial. Ce point de vue parait conforme à la conception de l'Univers cylindrique d'Einstein, car il résulte des formules de la solution d'Einstein que la courbure d'ensemble de l'Univers est déterminée par la quantité totale de matière existante; on trouve que le rayon $U$ de l'Univers est lié à la masse 


\section{I26 RELATIVITÉ GÉNÉR.ALISÉE ET GRAVITATION}

totale Mí de la matiere mondiale par la relation

$$
\mathrm{U}=\frac{\%}{4 \pi^{2}} \mathrm{M}
$$

( $\%$ est la constante de la formule 22, p. 109).

de sorte que, si par un miracle de la matière venait à ctre créée dans l'espace existant, le volume de cet espace augmenterait; la matière crée, en quelque sorte, l'espace qui la contient, et s'il n'y avait pas de matière, il n'y aurait pas d'Univers.

2" Une autre théorie, soutenue par M. Eddington, est la suivante: "Je préfère, dit M. Eddington, regarder la matière et l'énergic, non pas comme des facteurs produisant les différents degrés de courbure $\mathrm{d}$ I l'espace, mais comme des éléments de perception de cette courbure.

Cette manière de voir est en accord avec la solution de de Sitter, car dans cette solution il n'y a aucune relation cntre le rayon d'Univers et la masse totale de la matiere. L'Univers a une courbure naturelle; la matière n'est pas la cause de la courbure d'ensemble; elle correspond à des sortes de montagnes ou de rides, irrégularités lorales par lesquelles l'Univers est bien moins altéré que ne l'est la terre par le relief du sol. D'après cette théorie on pourrait concevoir un Univcrs vide de matière.

Dans cette hypothèse de la courbure naturelle, les lois générales sont des identifications de grandeurs physiques aver des grandeurs géonétriques, et nn peut les considérer comme: des définitions de grandeurs physiques. Si la courbure totale est ti. et si, de plus, le tenseur R'. est nul, nous disons qu'il y a le vide: cette structure géométrique se manifeate à nous snus un aspect particulier que nous appelons le vide. Si li courbure lolule est incore 4h, mis si R'. n'est plus 
nul, nous disons qu'il y a de l'énergie rayonnante; si enfin la courbure totale est différente de $4 \%$ nous sommes en présence d'une structure géométrique que nous appelons matière, et ce que nous appelons densité propre n'est autre chose que linvariant géométrique



Le ròle primordial est attribué à l'Espace-Temps, dont les divers degrés de courbure nous apparaissent sous des aspects que nos sens distinguent les uns des autres, et auxquels nous avons donné les noms de vide, rayonnement, matière. Cette manière de voir nous parail trés séduisante par sa logique et sa simplicité ${ }^{1}$.

C'est un retour à l'hypothèse d'un " substratum universel ", de l"éther par conséquent, mais cet éther est bicn différent de celui des anciennes théories.

L'espace vide de matière n'est pas anorphe, car la théoric de la relativité, qui ramène la mécanique et la physique à la géométrie de Riemann, prouve que l'Univers possède des proprićtés métriques en relation avec la matière présente ou avoisinante. Ces propriétés sont précisécs, dans chaque systeme de référence, par les valeurs des dix potenticls g:. du champ de gravitation et aussi, d'après une généralisation due à M. W Weyl, par les valcurs de quatre grandeurs qui constituent les composantes d'un quadrivecteur, le potentiel électromagnétique.

On doit, aussi bien dans l'hypothe'se cosmologique d'Einstein que dans celle de de Sitter, écarter la concep. tion que l'espace serait physiquement vide, au sens du néant absolu; il faut, non pas supprimer l'éther, mais donner une

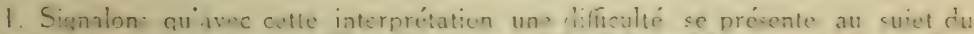
princine de moindre action. Toutefoi c the difficulte semble nouvoir di paraitre dans le csientom de la thérie d'Ein tein cont il ara quetion plus loin. 
forme nouvelle à la notion du substratum universel : l'éther de la relativité n'a rien de commun avec le milieu quasimatériel admis autrefois : c'est " un milieu privé de toutes les propriétés mécaniques et cinématiques, mais qui détermine les phénomènes mécaniques et électromagnétiq̣ues " (Einstein).

D'après Einstein, l'éther " détermine les relations métriques dans le continuum spatio-temporel, par exemple les possibilités de configuration des corps solides aussi bien que les champs de gravitation ".

Deux extensions successives de la théorie d'Einstein, dues à M. Weyl et à M. Eddington, paraissent apporter un complément fondamental. Grâce à l'union, en une géomśtrie unique, du champ de gravitation et du champ électromagnétique, on peut concevoir que les électrons (et par suite la matière) soient des états particuliers de la structure d'Univers, de l'éther au sens qu'on doit atiribuer aujourd'hui à ce mot.

En résumé l'espace possède des propriétés physiques, et l'on peut exprimer ce lait en disant qu' un "éther " existe. Mais " cet éther ne doit pas être conçu comme étant doué de la propriété qui caractérise les milieux pondérables, c'est-à-dire comme constitué de parties pouvant être suivies dans le temps: la notion de mouvement ne doit pas lui être appliquée" (Einstein).

On peut dire encore, avec M. Eddington, que l'éther est incapable de créer une division de l'Univers en espace et en temps. 


\section{CONCLUSIONS GENERALES}

La loi de la gravitation est maintenant connue: elle englobe toute la dynamique et bouleverse les anciennes conceptions. Jusqu à la découverte d'Einstein, non seulement on ignorait la loi exacte, mais on était bien loin de soupçonner la nature du champ de gravitation : on est certain aujourd'hui que ce champ est la manifestation du caractère non euclidien de la structure géométrique de l'Univers.

L'Univers est caractérisé, en chaque point-événement, par ses propriétés géométriques, liées à la présence ou au voisinage de la matière. L'espace n'est ni un vide amorphe, ni l'éther quasi-matériel de l'ancienne physique, et il ne doit pas être infini.

Le temps est l'aspect d'une des dimensions de la multiplicité quadridimensionnelle qui constitue l'Univers; il reste très mystérieux. S'il existe un système de référence privilégié auquel est lié un " temps d'Univers absolu" (hypothèse d'Einstein), ce temps absolu n'est pas, en toute rigueur, celui que nous percevons et que nous pouvons mesurer: pour nous il y a toujours, suivant la conception de Minkowski, union de l'espace et du temps: la division de l'Univers en espace et en temps n'est possible qu'en choisissant convenablement les coordonnées, et elle est relative à l'observateur. 
Toutefois, les phénomènes de la Nature ont un caractìre absolu, parce quils sont déterminés par des coincidences absolues dans l'Espace-Temps, des intersections de lign:s d'Univers. Il y a des réalités que la science peut atteindre: elles se traduisent par des lois qui s'expriment à l'aide d'équations intrinsèques, de relations tensorielles où tout systeme de coordonnées a disparu.

Cependant la théorie de la relativité ne remonte pas aux causes profondes des phénomènes: elle ne fait pas connaître la nature du substratum universel. C'est une description en langage mathématique, une interprétation géométrique des lois physiques et une magnifique synthèse de ces lois. C'est, dit M. Eddington, "la science de la structure et non celle de la substance".

La mécanique et la physique sont ramenées à la gécmétrie non-euclidienne de Riemann, ou plus exactement à la géométrie plus générale encore de MM. Weyl et Eddington (note 15); c'est là le fond de la théorie. Dans la géométrie, on groupe dans un tenseur des grandeurs inséparables les unes des autres, et l'annulation d'un tenseur (ou l'égalité de deux tenseurs) exprime une propriété intrinsèque de l'Univers. En mécanique et en physique, on forme des tenseurs arec des grandeurs que nous révele notre science expérimentale; la théorie de la relativité affirme que les tenseurs mécaniques et physiques fondamentaux doivent être égalés à certains tenseurs de la géométrie riemannienne.

Les tenseurs mécaniques et physiques sont égaux à des tenseurs gćométriques: cela ne saurait être mis en doute. mais comment faut-il comprendre ces égalités? S'agil-il d" "équations" ou d" "identités" ? La loi de la gravitation, celles de l'électromagnétieme sont-elles des conditions 
imposées par la Nature aux relations entre la matière et l'Espace-Temps, ou ne sont-elles que des identifications de l'aspect physique et de l'aspect géométrique des propriétés d'une même entité? Si l'Espace-Temps et la matière sont deux entités distinctes, les lois fondamentales sont des équations. Mais si l'on admet, arec M. Eddington, que les particules qui, en dernière analyse, constituent la matière ne sont autre chose qu'une structure géométrique d'Univers, la matière cesse d'être une entité primordiale, les tenseurs mécaniques et physiques deviennent des tenseurs géométriques vus sous un aspect relatif à notre interprétation de la Naturc, relatif à notre entendement.

Admettons cette conception. Est-ce dire que la loi de la gravitation est complètement subjective? Non pas, au fond, car il existe un théorème de géométrie, qui se traduit par les quatre identités dont nous avons parlé (p. 108); c'est là une propriété intrinséque de la multiplicité quadridimensionnelle qui constitue l'Univers, c'est une vérité objective. Mais la loi de conservation de l'impulsion-énergie et la loi de la gravitation sont des aspects subjectifs de cette vérité. L'homme a recherché ce qui, dansla Nature, se présente à ses yeux comme permanent: il a trouvé les lois de conservation de la masse, de l'énergie, de la quantité de mouvement; par synthèses successives, il a été conduit à identifier les grandeurs physiques qu'on peut grouper dans le tenseur matériel $T$. avec celles qui constituent un tenseur géométrique, le tonseur $R_{\text {. }}^{\prime}-\frac{1}{2}$ g. $R^{\prime}$ (éq. 25, p. 119) dont le théorème mentionné plus haut exprime précisément les propriétés de permanence: c'est la loi de la gravitation, d'où découle toute la dynamique. On ne peut pas prétendre que la Nature force la matière à suivre cette loi, car c'est nous qui définissons la 
matière de façon que cette loi soit satisfaite: ce que nous avons appelé tenseur matériel ou tenseur impulsion-énergie n'est pas autre chose qu'un certain tenseur d'Univers conservatif; notre loi de conservation ainsi que notre loi de la gravitation n'expriment, au fond, que des identités.

Nous avons fait allusion à deux généralisations successives de la théorie d'Einstein. Ces généralisations (IW eyl et Eddington) complètent la théorie d'Einstein sans l'altérer; leur intérêt est considérable. Partant des propriétés géométriques les plus générales que doit posséder un univers quadridimensionnel, M. Eddington a montré qu il doit exister deux catégories de propriétés qui correspondent à ce que les mathématiciens peuvent appeler la non-intégrabilité de la direction et la non-intégrabilité de la longueur; il doit en résulter, à nos yeux, deux catégories de phénomènes, deux champs de force de natures différentcs. La quadruple indétermination des coordonnées doit se traduire par quatre formules qui expriment une loi de conservation; mais ce n'est pas tout: l'indétermination du systeme de? mesures, c'est-à-dire l'indétermination de l'unité choisie en chaque point pour la mesure des intervalles, doit donner une autre loi de conservation.

C'est exactement ce que l'expérience nous révèle. Nous connaissons deux champs de force: le champ de gravitation et le champ électromagnétique; la conservation de limpulsion-énergie est une loi expérimentale qui sexprime par quatre équations; l'autre loi, bien connue, est celle de la conservation de l'électricité.

Quelle que puisse être, dans l'avenir, l'évolution dess idées, l'union de l'espace et du temps, l'inertie et la pesanteur de l'énergie. la loi de la gravitation. la dynamique de. la relativité, la courbure de l'Univers, les losis générales de 


\section{CONCLUSIONS GÉNÉRALES}

l'électromagnétisme sont des résultats, presque tous dus au génie d'Einstein, qui resteront acquis à la science.

La théorie actuelle pourra être retouchée ou plutôt complétée, surtout en ce qui concerne les hypothèses cosmologiques, la généralisation de la théorie d'Einstein et l'interprétation philosophique des lois. Mais ce qu'on peut affirmer, c'est qu'un retour en arrière, vers les idées encore enracinées dans quelques esprits, est une chose impossible. 


\section{APPENDICE}

\section{I. - RELATIVITÉ RESTREINTE.}

\section{Note 1 (p. 21).}

SUR LINVARIANCE DE LA DISTANCE SPATIALE DE DELX ÉVENLMLNIS SIMULTANÉS.

Soient $x: y: z 1, x: y: z$ : les coordonnées d'espace de deux érénements simultanés dans un premier système $\mathrm{S}$, et soient $x_{1} y_{1} z, x_{2} y_{z} z$ les coordonnées des deux mémes événements dans un second système $\mathrm{S}$. La distance spatiale est donnée par les équations:

$I^{2}=\left(x_{1}-x_{1}\right)^{2}-\left(y_{2}-y_{1}\right)^{2}+\left(z_{2}-z_{1}\right)^{2}$ dans le système $S$ $l^{\prime}=\left(x_{1}-x_{1}\right)^{2}+\left(y_{1}-y_{1}\right)^{2}+\left(z_{1}-z_{1}\right)^{2}$ dansle systeme $S^{\prime}$.

L'application des formules du groupe de Galilée donne $l=l$; le temps sélimine parce que, la simultanéité étant supposée absolue, les événements se produisent à la mème époque $t$ dans les deux systèmes.

\section{Note 2 (p. 24).}

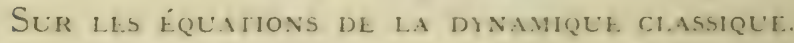

$m$ étant la masse d'un point matériel; $X, Y, Z$ et $X^{\prime}$, $Y^{\prime}, Z^{\prime}$ désignant les composantes de la force $F$ dans les



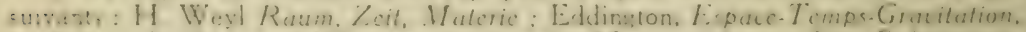

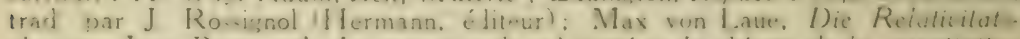
theorie: Jean Becqu-rel, Le principe de relaticité et la thiorie de la gracilation Gauthier-Villars, éditeur). 
systemes $S$ et $S^{\prime}$ non accélérés, les équations du mouvement sécrivent

$m \frac{d^{2} x}{d t^{2}}=\mathrm{X}, \quad m \frac{d^{2} y}{d t^{2}}=\mathrm{Y}, \quad m \frac{d^{2} z}{d t^{2}}=\mathrm{Z} \quad$ (système $\mathrm{S}$ )

$m \frac{d^{2} x^{\prime}}{d t^{2}}=X^{\prime}, \quad m \frac{d^{2} y^{\prime}}{d t^{2}}=Y^{\prime}, \quad m \frac{d^{2} z}{d t^{2}}=Z^{\prime} \quad$ (systeme S )

avec la relation



Les équations fondamentales ont la même forme dans les deux systemes; on peut les résumer par la relation vectorielle

$$
\overrightarrow{m \vartheta}=\vec{F} \quad \overrightarrow{(\because \text { vecteur accélération })}
$$

où tout systeme de coordonnées a disparu.

$$
\text { Note } 3 \text { (p. 31). }
$$

Sur la contraction de Fitzgerald-Lorentz.

Soient $l$ le trajet optique $\mathrm{OM}_{1}$ de la lumière entre la face semi-argentée de la lame $\mathrm{A}$ et le miroir $\mathrm{M}_{1}$; $l$ le trajet $\mathrm{OM}_{2}$ entre la lame et le miroir $\mathrm{M}_{2}$; si le bras $\mathrm{OM}_{1}$ est parallèle au mouvement absolu de la terre, le temps que met la lumière à aller de $\mathrm{O}$ au miroir $\mathrm{M}_{1}$ et à revenir en $\mathrm{O}$ est

$$
t_{1}=\frac{l^{\prime}}{c-v}+\frac{l^{\prime}}{c+v}
$$

Le temps employé pour parcourir le bras $\mathrm{OM}_{2}$, aller ct retour, est

$$
t_{2}=\frac{2 l}{\sqrt{c^{2}-v^{2}}}
$$


Pour que $t_{1}=t_{2}$, il faut et il suffit que

$$
\frac{l^{\prime}}{l}=\sqrt{1-\frac{v^{2}}{c^{2}}} .
$$

Note 4 (p. 37).

REMARQue sUR LA MESURE DU TEMPS.

En disant que les observateurs ont des horloges étalons identiques, nous supposons qu'ils mesurent le temps en prenant comme étalon de temps la période d'un même phénomène, en choisissant un phénomène qui ne snit pas déterminé par des conditions spéciales à un système particulier: par exemple, un pendule ne pourra pas servir d'étalon universel, parce que sa période d'oscillation est déterminé par la pesanteur; mais on pourra adopter la période d'une radiation émise par un corps et prendre pour unité de temps, dans tous les systèmes, un même multiple de cette période.

Note 5 (p. 39).

LE GROUPE DE LORENTZ.

Soit un même événement noté $x_{v}, y_{1}, z$, tu par lés olsservateurs du systeme $\mathrm{S}$ et noté $x, y, z, i$ par lés olsservateurs du système $\mathrm{S}$ en translation uniforme avec ha vitesse $v$ par rapport à $\mathrm{S}$.

Cherchons lesfonctions $f, f, f \rightarrow f$, satisfaisant aux relations

$$
\begin{aligned}
& x^{\prime}-x_{0}^{\prime}=f_{1}\left(x-x_{0,} y-y_{0,} z-z_{0, t}-t_{11}\right) \\
& \cdot t^{\prime}-\dot{t}_{0}^{\prime}=f_{4}\left(x-x_{0,} \cdot y_{0}, z-z_{0}, t-t_{0}\right) .
\end{aligned}
$$

Si l'on suppose la combinaison de l'espace et du tempras homogène, ces formules doivent s'appliquer quel que soit 
l'événement de référence $\left(x_{i}, y_{i}, z, t_{0}\right)$ ou $\left(x^{\prime}, y_{\prime \prime}^{\prime}, z^{\prime}, t^{\prime}\right), \mathrm{et}$ l'on trouve aisément la forme des fonctions: considérons trois événements (indices $0,1,2$ ), nous aurons

$$
\begin{aligned}
& x_{1}-x_{0}=f_{1}\left(x_{1}-x_{0}, y_{1}-y_{10}, z_{1}-z_{1}, t_{1}-t_{0}\right), \\
& x_{1}-x_{1}=f_{1}\left(x_{2}-x_{1}, y_{2}-y_{1}, z_{2}-z_{1}, t_{2}-t_{1}\right), \\
& x_{2}-x_{0}=f_{1}\left(x_{2}-x_{0}, y_{2}-y_{11}, z_{2}-z_{11}, t_{2}-t_{0}\right) .
\end{aligned}
$$

D'où

$f_{1}\left(x_{2}-x_{1}\right.$, etc $\left.\ldots\right)=f_{1}\left(x_{-}-x_{1}\right.$, etc... $)+f_{1}\left(x_{1}-x_{n}\right.$, etc...). éguation fonctionnelle qui montre que $f_{1}$ cst une fonction lincaire el homogène de ses arguments; il en est de même de $f_{: 3}, f_{:}, f_{4}$ 。

Prenons maintenant comme premier événement l'émission d'un signal lumineux en $\mathrm{O}$ et $\mathrm{O}$ à l'origine des temps; au bout du temps $t$, pour l'observateur du systeme $\mathrm{S}$, le signal lumineux est sur la surface de la sphère du système $\mathrm{S}$.

$$
x^{2}+y^{2}+z^{2}-c^{2} t^{2}=0 \text {. }
$$

la vilcsse de la lumière ćtant une constante universclle, pour lobservateur du systeme $\mathrm{S}^{\prime}$, le signal est au bout du temps $t$ sur la sphère du système $S$

$$
x^{2}+y^{2}+z^{2}-c^{2} t^{2}=0 \text {. }
$$

Si $x, y, z, t, x^{\prime}, y, z, t$ sont les coordonnées d'un méme apparcil qui reçnit le signal (second événement), on a

$$
x^{2}+y^{2}+z^{2}-c^{2} t^{2}=\lambda\left(x^{2}+y^{2}-z^{2}-c^{2} t^{2}\right)=0 \text {. }
$$

Les lois des phenomenes ne deiant pas changer quand nn passe de $\mathrm{S}$ ì $\mathrm{S}$ el réciproquement, on a nécessairement $\lambda=1 \mathrm{et}$

(5-1) $x^{2}+y^{2}+z^{2}-c^{2} t^{2}=x^{2}+y^{2}+z^{2}-c^{2} t^{2}$. 
La disposition d'axes choisie exige que :
(5-2)
quels que soient $y$ et $z$ on ait à la fois $x,=0$ et $x=$ tt

$$
\begin{aligned}
& x, z \text { et } t \quad-\quad y^{\prime}=0 \text { et } y=0 \\
& x, y \text { et } t \quad-\quad z^{\prime}=0 \text { et } z=0 .
\end{aligned}
$$$$
x, y \text { et } t
$$

Les relations linéaires et homogènes qui donnent $x, y^{\prime}$, $z, t$ en fonction des $x, y, z, t$ contiennent dans le cas général 16 coefficients fonctions de $v$; avec la disposition envisagée, en vertu des conditions (5-2) il ne reste que 7 coefficients; on les calcule aisément d'après l'iilcntilé (5-1) et l'on trouve les formules de Lorentz.

$$
\text { Note } 6 \text { (p. 44). }
$$

\section{LA COMPOSITION DES VITESSES.}

Différencions les équations de Lorentz

$$
\begin{gathered}
d x=-\frac{1}{\alpha}\left(d x^{\prime}+v d t^{\prime}\right), \quad d y=d y^{\prime}, \quad d z=d z^{\prime}, \\
d t=\frac{1}{x}\left(d t^{\prime}+\frac{v}{c^{2}} d x^{\prime}\right) .
\end{gathered}
$$

Divisant les trois premières de ces équations par la dernière, nous obtenons

(6-1)

$$
\begin{aligned}
& v_{x}^{\prime \prime}=\frac{d x}{d t}=\frac{v+v_{x}^{\prime}}{1+\frac{v v_{x}^{\prime}}{c^{2}}}, \\
& v_{y}=\frac{d y}{d t}=\% \frac{v_{y}^{\prime}}{1+\frac{v v_{x}^{\prime}}{c^{\prime}}}, \\
& v_{z}=\frac{d z}{d t}=2 \frac{v_{z}^{\prime}}{1+\frac{v v_{x}^{\prime}}{c^{2}}} .
\end{aligned}
$$


En particulier si, comme nous l'avons supposé dans le texte, $v$ est parallèle à $v$, on a

$(6-2)$

$$
v^{\prime \prime}=\frac{v+v}{1+\frac{v v}{c^{2}}} .
$$

Note 7 (p. 52).

CONIRACIION DES LONGUELRS ET DILAIAIION DU TLMPS.

Snient $x_{1}, x_{2}$ et $x_{1}, x_{-}$les abscisses des deux extrémités de la tige dans les systemes $\mathrm{S}$ et $\mathrm{S}$; la premiere des formules de Lorentz

$$
x^{\prime}=\frac{1}{x}(x-v t)
$$

appliquée aux deux points extrémités de la tige, à un míme instant $t$ du système $\mathrm{S}$, donne

$$
\text { (7-1) } x_{2}-x_{1}^{\prime}=\frac{1}{2}\left(x_{2}-x_{1}\right) \text { ou } l=l^{\prime} \text {. }
$$

$D^{\prime}$ autre part, considérons une horloge du systeme $S^{\prime}$ et deux événements infiniment voisins se produisant sur cette horloge; nous avons : invariant $d s^{2}=c^{2} d t^{2}=c^{2} d t^{2}-d x^{2}$ avec $d x=v d t$.

D'où
$(7-2)$
$d t=\frac{1}{\%} d t$

Soit maintenant une tige infiniment courte dirigée parallèlement à la viiesse, immobile dans le système $\mathrm{S}$ et de longueui $d x^{\prime}$ dans ce système; considérons deux événements infiniment voisins concernant cette tige; d'après (7-1) 
et (7-2), l'olsservateur du système $\mathrm{S}$ mesure :

$$
d x=\% d x^{\prime} \quad \text { et } \quad d t=\frac{1}{\%} d t .
$$

On a donc
$(7-3)$
$d x d t=d x^{\prime} d t^{\prime}$.

INVARIANCE. DE L'HYPERVOLUMIE D'UNIVERS, - Avec des tiges de !ongueurs au repos $d x, d y^{\prime}, d z$ dirigées parallèlement aux axes, formons un parallélipipède rectanglc, immobile dans $\mathrm{S}^{\prime}$; soit $d t$ un intervalle de temps infiniment court marqué par une horloge au centre de parallélipipède; comme, avec notre choix d'axes, $d y=d y^{\prime}$ et $d z=d z$, nous obtenons
$(7-4)$
$d x d y d z d t=d x^{\prime} d y^{\prime} d z^{\prime} d t^{\prime}$

ou cncore en prenant comme coordonnée de temps la longueur $u=c t$ $d x d y d z d u=d x^{\prime} d y^{\prime} d z^{\prime} d u^{\prime}$.

Note 8 (p. 57).

SUR LE TEMPS PROPRE.

Considérons deux mobiles $\mathrm{M}_{1}$ et $\mathrm{M}_{\text {: en }}$ coïncidence al)solue aux événements $\mathrm{A}$ et $\mathrm{B}$, et ayant, entre ces érénements, des lignes d'Univers différentes. Supposons que $\mathrm{M}_{1}$ soit en translation uniforme; $\mathrm{M}_{2}$ a alors nécessairement subi une accélération entre les deux érénements considérés. Repérons les événements dans un système $\mathrm{S}$ (en translation uniforme) lié à $\mathrm{M}_{1}$.

Prenons deux époques $t$ et $t+d t$ du temps du systeme $\mathrm{S}$, comprises entre les épogues $t_{\mathrm{A}}$ et $t_{\mathrm{B}}$ auxquelles se pro- 
duisent, toujours dans le système $S$ lié à $\mathrm{M}_{1}$, les événements A et B. Aux époques $t$ et $t+d t$, le second mobile $M_{2}$ est repéré $x, y, z, t ; x+d x, y+d y, z+d z, t+d t$ dans le système $\mathrm{S}$; ces coordonnées déterminent, sur la ligne d'Univers de $\mathrm{M}_{2}$, deux événements infiniment voisins dont lintervalle est $d s$; on a

$$
d s^{2}=-d x^{2}-d y^{2}-d z^{2}+c^{2} d t^{2}
$$

mais on a aussi

$$
d s=c d t
$$

par conséquent

(8-1) $d s=\% d t, \quad$ ou $\quad d t=\% d t \quad\left(\%=\left(1-\frac{v^{2}}{c^{2}}\right)\right.$.

Les mobiles $M_{1}$ et $M_{2}$ étant en coïncidence absolue aux événements $\mathrm{A}$ et $\mathrm{B}$, nous obtenons, par intégration,

$$
\int_{\mathrm{A}}^{\mathrm{B}} d t=\int_{t_{\mathrm{A}}}^{t_{\mathrm{B}}}{ }_{0} d t
$$

Plus le mouvement de $M_{2}$ aura été accéléré, plus, par conséquent, les vitesses par rapport à $M_{1}$ seront grandes puisque la durée totale $t_{B}-t_{A}$ est fixe, et plus le temps propre total sera court.

En d'autres termes, entre deux événements déterminés, la plus longue ligne d'Univers est celle qui correspond au mouvement rectiligne et uniforme.

\section{Note 9 (p. 59). \\ LA rol Dinertie.}

Une fonction a une variation nulle lorsqu elle passe par un 
minimum ou par un maximum. Donc, pour la ligne géométrique la plus courte on $a \vdots \mid d l=0$, et pour la ligne d'Univers la plus longue on a

$$
\Xi \int d s=0 \text {. }
$$

Dans un cas comme dans l'autre, l'énoncé sous forme de loi d'action stationnaire est le même. La formule (9-1) est l'expression intrinsèque, indépendante de tout système de coordonnées, de la loi d’inertie.

\section{Note 10.}

\section{I. - Le champ électromagnétique.}

Dans un système de référence $\mathrm{S}$, soient au point $x, y, z$ et à l'instant $t: \mathrm{X}, \mathrm{Y}, \mathrm{Z}$ les composantes de la force électrique. L. M, N les composantes de l'induction magnétique, l' la densité de charge multipliée par $4 \pi, u_{x}, u_{y}, u_{z}$ les composantes de la vitesse de la charge supposée en mouvement (courant de convection). Les équations de Maxwell-Lorentz. s'écrivent

(10-1)




$(10-2)$

$$
\begin{gathered}
\frac{1}{c}\left(\frac{\partial \mathrm{X}}{\partial t}+i w_{x}\right)=\frac{\partial \mathrm{N}}{\partial y}-\frac{\partial \mathrm{M}}{\partial z} \\
\frac{1}{c}\left(\frac{\partial \mathrm{Y}}{\partial t}+i w_{y}\right)=\frac{\partial \mathrm{L}}{\partial z} \frac{\partial \mathrm{N}}{\partial x} \\
\frac{1}{c}\left(\frac{\partial Z}{\partial t}+i w_{z}\right)=\frac{\partial \mathrm{M}}{\partial x}-\frac{\partial \mathrm{L}}{\partial y} \\
\text { Div. }(\mathrm{X}, \mathrm{Y}, \mathrm{Z})=\frac{\partial \mathrm{X}}{{ }_{x}}+\frac{\partial \mathrm{Y}}{\partial y}+\frac{\partial \mathrm{Z}}{\partial z}=\mathrm{I} .
\end{gathered}
$$

Dans un système de référence $\mathrm{S}^{\prime}$ animé de la vitesse $v$ par rapport à $S$, ces équations doivent être remplacées par des équations de même forme. Adoptons la disposition d'axes précédemment considérée; le calcul montre que ces équations restent les mêmes si les nouvelles grandeurs (lettres accentuées) sont liées aux anciennes par les relations suivantes:

1" Les formules de Lorentz pour les transformations d'espace et de temps;

2 Les formules (6-1) de composition des vitesses pour les w;

3" Les formules suivantes, pour la transformation des grandeurs électriques et magnétiques.

$\mathrm{X}^{\prime}=\mathrm{X}$

$(10-3)$

$(10-4)$
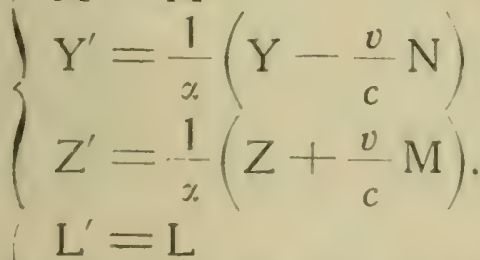

$\mathrm{M}^{\prime}=\frac{1}{\%}\left(\mathrm{M}+{ }_{c}^{v} \mathrm{Z}\right)$

( $N^{\prime}=\frac{1}{\%}\left(N-\frac{v}{c} Y\right)$. 


$$
f^{\prime \prime}(10-5) \quad \Gamma^{\prime}=\frac{1}{x}\left(1-\frac{i u_{x}^{\prime}}{c^{2}}\right) \Gamma^{\prime} .
$$

a) Cette dernière formule donne immádiatement un résultat fondamental.

Soit e la charge de l'élément de volume d'espace, on a

$$
\mathrm{P}=\frac{4 \pi c}{d x d y d z}, \quad \mathrm{P}^{\prime}=\frac{4 \pi e^{\prime}}{d x^{\prime} d y^{\prime} d z^{\prime}},
$$

de (10-5) on déduit

$e d x d y d z d t=e d x^{\prime} d y d z d t$ ou $e=e$ d'apres $(T-4)$.

La charge électrique est un intariant.

b) Les formules $(10-31,(10-4)$ montrent qu'un champ électrique et un champ magnétique nont pas d'existence absolue. Par exemple, ce qui est un pur champ magnétique dans un système est un champ mixte (électrique et magnétique) dans un autre systeme : cecifait comprendre laction d'un champ magnétique sur une charge en mouvement. car, alors que dans le système de lobservateur, il existe un pur champ magnétique, dans le sustème do la charge. it règne un champ électrique qui agit sur elle. On retrouve facilement, d'après ces formules, la loi de Biot et Savart et la loi de l'induction, qui ne subissent aucune correction dans la théorie nouvelle.

c) Appliquées aux ondes lumineuses, les formules de transformation permettent d'établir la théorie exacte de leffet Doppler et de laberration de la lumiere. Les anciennes formules ne constituent que des approximations; les formules exactes sont : 
$(10-6)$

$$
1-\frac{v}{c} \cos \frac{7}{7}
$$

$(10-7)$

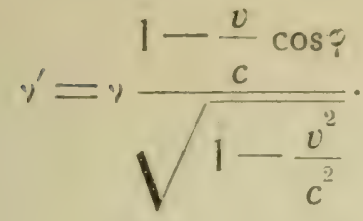

$$
\cos \theta-\frac{v}{c}
$$

$$
1-\frac{v}{c} \cos q
$$

" est la fiéquence propre de la source et "' la fréquence apparente pour l'observateur. ¿ est l'angle de la vitesse $t$ et du rayon lumineux dans le systeme $\mathrm{S}$ de la source, est l'angle de la vitesse $v$ et du rayon reçu par l'o!sservateur.

L'énergie lumineuse se transforme suivant la même loi que la fréquence.

(10-8)

$$
W^{\prime}=\mathbb{W} \frac{1-\frac{v}{c} \cos ?}{\sqrt{1-\frac{v^{2}}{c^{2}}}} .
$$

On trouve enfin l'expression de la pression de la lumière sur un réflecteur intégral, animé de la vitesse $v$ par rapport à l'observateur.

$$
\text { (10-9) } \quad p=2 \frac{\mathrm{A}^{2}}{8 \pi} \frac{\left(\cos s-\frac{v}{c}\right)^{2}}{1-\frac{v^{2}}{c^{2}}} \text {. }
$$

A étant l'amplitude des ondes, pour l'ohserrateur. 
II. - Dynamique de la relativité.

$1^{n}$ LA MASSE EST FONCTION DE L.A VITESSE. - Dans un système $S$, un point matériel est supposé en mouvement avec la vitesse $v$ à l'instant $t$.

Introduisons un second système $\mathrm{S}^{\prime}$ animé de la vitesse v par rapport à $\mathrm{S}$. Dans $\mathrm{S}^{\prime}$, à l'instant considéré, le mobile a une vitesse nulle; pendant le temps infniment court qui suit, nous pouvons dans le système $\mathrm{S}^{\prime}$ appliquer les équittions de la dynamique classique puisque le mobile part du repos dans ce système.

Soient $m$ la masse initiale ou masse au repos du point matériel; $F_{x}^{\prime}, F_{y}^{\prime}, F_{z}$, les composantes, mesurées par un observateur du système $S^{\prime}$, de la force que subit ce point: nous avons
(10-10)
$m_{11} \frac{a^{2} x^{\prime}}{d t^{2}}=F_{*}^{\prime}$
$m \frac{d^{2} y^{\prime}}{d t^{\prime 2}}=F_{s}^{\prime}$
$m \frac{d^{2} z^{\prime}}{d t^{\prime}}=\mathrm{F}_{z}$

Pour avoir les équations de la dynamique dans le système $\mathrm{S}$, il faut chercher comment se transforment $\frac{d^{2} x^{\prime}}{d t^{-}} d^{\prime \prime} u^{\prime}$. $\frac{d^{2} z^{\prime}}{d t^{\prime 2}}$ et $F_{x}^{\prime}, F_{y}^{\prime}, F_{z}^{\prime}$ en fonction des mesures faites dans le système S.

Adoptons la disposition d'axes habituelle, en prenant $O_{x}$ et $\mathrm{O} x$ ' parallèles à $\imath$. Les formules de Lorentz permeitent de calculer $\frac{d^{2} x}{d t^{\prime 2}} \cdots$ en fonction de $v$ et de $\frac{d^{2} x}{d t^{2}} \cdots$ en tenant compte de ce que $\frac{d x}{d t}=t$ à linstant $t$ considéré.
On trouve
(10.11) $\frac{d^{2} x^{\prime}}{d t^{\prime 2}}=\frac{1}{2^{3}} \frac{d^{2} x}{d t^{2}}$
$\frac{d^{2} u^{\prime}}{d !^{\prime 2}}=\frac{1}{u^{2}} d d^{2} u$
$\frac{d^{2} z}{d i^{2}}=$
$\frac{d l^{2} z}{3 !}$ 
Pour obtenir la transformation des $F_{x}^{\prime}, F_{y}^{\prime}, F_{z}^{\prime}$ nous considérons le cas d'une force électrique; supposons que dans $S$ règne un champ électrique $X, Y, Z$; pour les observateurs de ce systeme, il s'exerce sur une particule de charge e une force

$$
\mathrm{F}_{x}=\mathrm{eX}, \quad \mathrm{F}_{y}=\mathrm{eY} \quad \mathrm{F}_{z}=\mathrm{eZ} .
$$

Appliquons (10-3), en y faisant $\mathrm{M}=\mathrm{N}=\mathrm{O}$, et remarquons que $c$ est un invariant: nous obtenons

$$
(10-12) \quad \mathrm{F}_{x}^{\prime}=\mathrm{F}_{x}, \quad \mathrm{~F}_{y}^{\prime}=\frac{1}{\%} \mathrm{~F}_{y}, \quad \mathrm{~F}_{z}^{\prime}=\frac{1}{\%} \mathrm{~F}_{z} .
$$

substituant dans (10-10) les valeurs de l'accélération (10-11) et de la force (10-12) il vient
$(10-13)$
$\frac{m_{0}}{x^{3}} \frac{d^{2} x}{d t^{2}}=F_{x}$
$\frac{m}{\%} \frac{d^{2} y}{d t^{2}}=F_{y}$

$$
\frac{m_{11}}{d^{2} z} \frac{\mathrm{F}_{z}}{d t^{2}}
$$

Bien qu'établies dans le cas particulier de la force éicetrique ces équations s'appliquent à une force quelconque, car si une force mécanique (par exemple la tension d'un ressort) fait équilibre à l'action exercée par un champ électrique, c'est un fait sur lequel tous les observateurs doivent se trouver d'accord. Il est donc nécessaire que les composantes de la force mécanique se transforment comme celles de la force électrique.

On trouve ainsi une masse longitudinale $\frac{m_{0}}{\alpha^{3}}$ et une masse transversale $\frac{m_{0}}{\sim}$, la masse étant définie comme coefficient d'inertie.

Mais les équations (10-13) peuvent s'écrire: 


$$
\frac{d}{d t}\left(\frac{m_{0}}{\%} \frac{d x}{d t}\right)=\mathrm{F}_{x}, \quad \frac{d}{d t}\left(\frac{m_{u}}{\%} d y\right)=\mathrm{F}_{y}, \quad \frac{d}{d t}\left(\frac{m_{u}}{2} \frac{d z}{d t}\right)=\mathrm{F}_{z}
$$

sous cette forme symétrique, la restriction due au choix particulier des axes est levée; les équations sont absolument générales. $\mathrm{F}_{x} d t, \mathrm{~F}_{y} d t, \mathrm{~F}_{z} d t$ sont les composantes de l'impulsion $d \mathrm{G}$; on a donc, en intégrant et prenant la quantité de mouvement nulle au repos

$$
\frac{m_{0}}{\alpha} \vec{v}=\overrightarrow{\mathrm{G}}
$$

la masse définie comme capacité d'impulsion est $\frac{m}{\%}$.

2 L'INERTIE DE L'ÉNERGIE. - Multipliant les équations (10-14) par $d x, d y, d z$ et ajoutant, on obtient

$$
d\left(\frac{m_{11}}{x} c^{2}\right)=d\left(m c^{2}\right)=F_{x} d x+F_{y} d y+F_{z} d z=a W
$$

$d W$ étant l'énergie fournie au point matériel.

On a donc
(10-16)

$$
m c^{2}=W+C^{t e} .
$$

La variation de masse est proportionnelle à la variation d'énergie cinétique.

a) Masse de l'énergie rayonnante. - Considérons un train d'ondes planes tombant normalement sur une surface noire S. L"énergie $\lesssim \mathbb{W}$ absorbée pendant le temps シ/ exerce

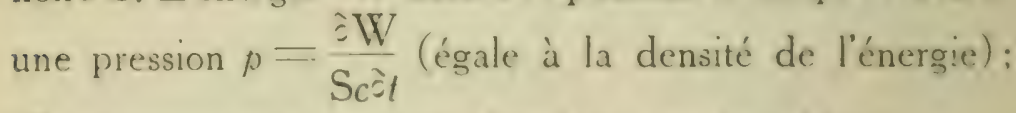
elle communique au corps absorbant une impulsion

$$
: \mathrm{G}=p \mathrm{~S}: t=\frac{\Sigma W \mathrm{~W}}{c} \text {. }
$$


L'énergie rayonnante $\mathbb{W}$ possède donc une quantité de

mouvement $G=\frac{W}{W}$ c'est-à-dire une masse (capacité d'impulsion)
nulle.

b) Un corps qui rayonne éprouve une perte de masse égale à la masse $\frac{\mathrm{W}}{c^{2}}$ de l'énergie rayonnée $\mathrm{W}$. - Prenons un cas simple: une lame plane normale à $O x$ rayonne par ses deux faces, avec la même intensité, des ondes planes se propageant de part et d'autre normalement à son plan.

Dans un système de référence $\mathrm{S}$ par rapport auguel elle était immobile avant de rayonner, la source envoie, de part et d'autre, des quantités de mouvement électromagnétiques égales et opposées; elle reste donc immobile. Soit W une certaine quantité d'énergie rayonnée $\left(\frac{W}{2}\right.$ de chaque côté), mesurée par un observateur du systeme S.

Pour un second observateur $\mathrm{S}$ animé, par rapport à $h$ source, d'une vitesse $v$ parallèlement à $O x$, l'énergic se transforme d'après (10-8) (où $₹=0$ et $₹=-$ ).

Cet observateur mesure

$$
W^{\prime}=\frac{1}{\%} \frac{W}{2}\left(1-\frac{v}{c}\right)
$$

pour l'énergie envoyée dans la direction et le sens de $v$ et

$$
\mathbb{W}=\frac{1}{\sigma} \frac{W}{2}\left(1+\frac{v}{c}\right)
$$

dans le sens opposé. 
La quantité de mouvement qui s'est propagée, pour S', dans le sens de $v$ est

$$
(10-17) \quad \frac{\mathrm{IV}}{c}-\frac{\mathrm{W}}{c}=\frac{1}{\%} \frac{\mathrm{W}}{c^{2}}(-v)
$$

$(-v)$ est la vitesse de la source pour $S^{\prime}$. D'après la conservation de la quantité de mouvement, $\frac{1}{\%} \frac{W}{c^{2}}(-v)$ est la quantité de mouvement perdue par la lame. Comme la vitesse n'a pas changé, cette variation provient d'une variation de masse de la lame

$$
\frac{\text { I }}{c^{2}} \text { pour l'observateur } \mathrm{S}^{\prime}
$$

et $\frac{\mathrm{W}}{c^{2}}$ pour l'observateur $\mathrm{S}$ immobile par rapport à la source.

La lame a donc éprouvé une perte de masse au repos IV

précisément égale à la masse de l'énergie rayonnée.

c) L'énergie potentielle totale d'un électron est égale d̀ sa masse au repos multipliée par $c^{2}$. (M. Langevin). Assimilons l'électron à une sphère de rayon a possédant une charge superficielle $e$.

L’énergie potentielle du champ électrostatique $h$ est :

$$
\begin{aligned}
& \mathrm{WV}_{1}=\frac{1}{8 \pi} \int^{2} \iint^{2} h^{2} d \mathrm{~V}=\frac{e^{2}}{8 \pi} \iiint^{0} \frac{1}{r} d \mathrm{~V} \\
& =\frac{e^{2}}{8 \pi} \int_{a}^{\infty} \int_{0}^{\infty} \int_{i}^{2-} \frac{\sin (0)}{r^{2}} d r d \theta d s=\frac{c^{2}}{2 a}
\end{aligned}
$$


Soient $==\frac{c}{4 \pi a^{2}}$ la densité superficielle de charge, $p$ la pression de Poincaré, nécessaire à admettre pour expliquer que la charge ne se dissipe pas. La pression $p$ fait équilibre à la tension $2 \pi \tau^{2}$ résultant de la répulsion mutuelle des éléments qui composent la charge.

On a donc

$$
p=2 \pi \sigma^{2}=\frac{e^{2}}{8 \pi a^{i}} .
$$

Il en résulte une énergie potentielle $W_{\text {: égale au pro- }}$ duit de $p$ par le volume de l'électron.

$$
\mathrm{W}_{2}=\frac{4}{3} \pi a^{3} p=\frac{1}{6} \frac{e^{2}}{a} .
$$

L'énergie potentielle totale de l'électron au repos est ainsi

$$
\text { (10-18) } \quad W=W_{1}+W_{2}=\frac{2}{3} e^{e^{2}} \text {. }
$$
Or la masse de l'électron est $\frac{1}{c^{2}} \frac{2}{3} \frac{e^{2}}{a}$; on a par suite
$m c^{2}=\mathrm{W}$.

Généralisation. - Dans tous les cas où l’on peut calculer l'énergie totale $\mathbb{W}$ d'un système, on la trouve égale à $m c^{2}$. On est donc conduit à généraliser et à donner les lois énoncées page 66.

$3^{n}$ L'IMPULSION D'UNIVERS. - Soit d: l'élément de temps propre d'un point matériel $\left(d_{0}=\frac{1}{c} d s\right)$. Les dérivées $\frac{d x}{d t}, \quad \frac{d y}{d t}, \quad \frac{d z}{d t}, \quad \frac{c d t}{d t}$ 
se transforment comme les composantes $d x, d y, d z$, cdl d'un déplacement élémentaire, puisque $d$ - est un invariant. Ce sont donc les composantes d'un quadrivecteur, la vitesse généralisée.

Multiplions par linvariant m., (masse au repos) les composantes de ce quadrivecteur; nous avons les composantes de l'impulsion d'Univers.

$$
m_{11} \frac{d x}{d \tau}, \quad m_{u} \frac{d y}{d \tau}, \quad m_{1} \frac{d z}{d \tau}, \quad m \cdots c \frac{d t}{d \tau}
$$

qui s'écrivent, puisque $d=z d t$ et $m=\frac{m}{\%}$

(10-19) $m \frac{d x}{d t}, \quad m \frac{d y}{d t}, \quad m \frac{d z}{d t}$. mc.

Les trois premieres composantes (composantes d'espace) sont les composantes de la quantité de mouvement: Ia quatrième (composante de temps) est l'énergie divisée par c.

La conservation de la quantité de mouvement et la conservation de l'énergie qui, pour un système de points matériels isolé, s'écrivent :

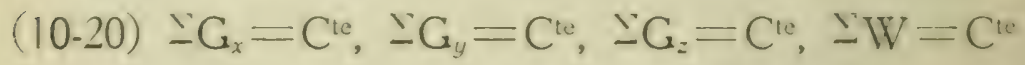

se résument maintenant dans l'affirmation que la sommine des iccleurs impulsions d'Livicrs, somme entendue au sems géométrique reste constante dans un systeme matéricl isolé. Elle est indépendante du système de référence, alors que la quantité de mouvement et l'énergie varient d'un système de référence à un autre. Le principe de la conservation de l'impulsion d'Univers a seul un sens absolu. 


\title{
RELATIVITÉ GÉNÉRALISÉE
}

\author{
Note 11. \\ Les TENSEURs.
}

I" TR.INSFORMATION DU DÉPLACEMENT ÉLÉMENTAIRE. - Passons d'un système de coordonnées $\left(x_{1}, x_{2}, x_{i}, x_{i}\right)$ à un autre $\left(x_{1}^{\prime}, x_{2}^{\prime}, x_{i}^{\prime}, x_{i}^{\prime}\right)$ l'élément de ligne se transforme d'après les quatre équations

$$
d x_{1}=\frac{\partial x_{1}^{\prime}}{\partial x_{1}} d x_{1}+\frac{{ }^{\prime} x_{i}^{\prime}}{\partial x_{2}} d x_{2}+\frac{\lambda^{\prime} x_{1}}{\partial x_{3}} d x_{3}+\frac{{ }^{\prime} x_{1}}{\partial x_{i}} d x_{i}
$$

qu'on résume sous la forme abrégée

$$
(11-1) \quad d x_{\tau}^{\prime}=\sum_{\nu}^{1} \frac{\partial x_{\sigma}^{\prime}}{\partial x_{\nu}} d x
$$

F étant le même indice dans les deux membres et la sommation étant faite, pour chaque indice 5 , en remplaçant" successivement par 1, 2, 3, 4 .

2" QU.ADRIVECTEURS. - Tout groupe de quatre quantités $\mathrm{A}^{\prime}$ qui se transforment suivant la même loi que les $d x$,

$$
\text { (11-2) } \quad \mathrm{A}^{\prime \sigma}=\sum \frac{\partial x_{\sigma}^{\prime}}{\lambda_{x}} \mathrm{~A}^{\prime \prime}
$$

constitue un quadrivecteur ou tenseur de premier ordre conlrevariant. On met l'indice en haut (sauf pour $d x$, qui est cependant contrevariant). 
Tout groune de quatre quantités $B$. (indice en bas) quui se transforment suivant la loi

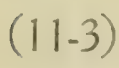

$$
\mathrm{B}^{\prime}=\sum_{\nu} \frac{\partial_{x}}{\partial_{x_{i}}^{\prime}} \mathrm{B}
$$

constitue un quadrivecteur ou tenseur de premicr ordre covariant.

On voit facilement que

$$
\text { (11-4) } \quad \Sigma A B= \pm A B=\text { invariant. }
$$

Un invariant, appelé aussi sculaire. est un tenseur d'ordre nul.

Remarque. - La sommation est faite par rapport à l'indice qui figure deux fois sous le signe - . Cet indice n'a pas de signification propre puisque dans l'expression complète d'une méme composante on lui donne successivenent les valeurs 1, 2, 3, 4: on l'appelle indice muct. La lettre qui le désigne peut être à volonté remplacée par une autre pourvu que la nouvelle lettre ne figure pas déjà dans le terme considéré. Dans la suite nous supprimerons le il sera sous-entendu quion doit toujours sommer par rapport aux indices muets, faciles à reconnaitre d'après ce qui vient d'être dit.

$3^{\prime \prime}$ TENSEURS DORDRE SUPERIEUR. - Scize grandeurs qui se transforment suivant la loi

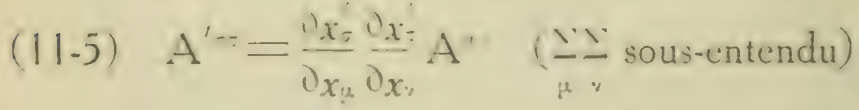

sont les composantes d'un tensicur du second urde contrevariant. 
Seize grandeurs qui se transforment suivant la loi

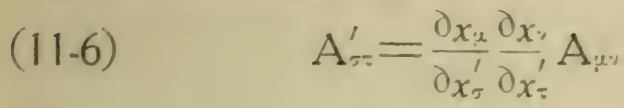

forment un tenscur du second ordre covariant.

On généralise aisément pour définir des tenseurs d'ordre $n$. Un tenseur d'ordre $n$ possède $4^{n}$ composantes (dans une multiplicité à 4 dimensions). Un tenseur qui participe à la fois des deux modes de transformation est dit mixte : il est contrevariant vis-à-vis de certains indices et covariant vis-à-vis des autres.

$$
\text { Ex.: } A_{\sigma=}^{\prime ?}=\frac{\partial x_{\rho}^{\prime}}{\partial x_{\varepsilon}} \frac{\partial x_{\mu}^{\prime}}{\partial x_{\sigma}^{\prime}} \frac{\partial x_{\nu}}{\partial x_{\tau}^{\prime}} A_{\%}^{\varepsilon}
$$

Un tenseur tel que $\mathrm{A}_{1,5} \ldots=\mathrm{A}_{\ldots} \ldots$ est dit symétrique

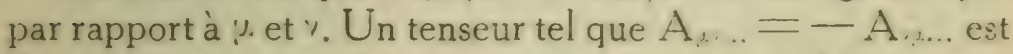
dit symétrique gauche par rapport à \% et \% Un tenseur symétrique gauche d'ordre 2 possède six composantes distinctes (au signe près); il n'y a pas de tenseur symétrique gauche d'ordre supérieur à quatre (du moins dans une multiplicité quadridimensionnelle).

$4^{\circ}$ MULTIPLiCATION. - Si l'on multiplie deux à deux les composantes d'un tenseur d'ordre $n$ et celles d'un tenseur d'ordre $n$, on obtient un tenseur d'ordre $n+n$ '.



5" CONTRACTION. - Partant d'un tenseur mixte, nn peut former un tenseur d'ordre inférieur de deux unités en égalant un indice de caractère covariant et un indice contrevariant. Ex. : soit $\mathrm{A}_{\text {;.. }}$; imposons la condition $z=-$, nous obtenons $A_{1.5}^{5}$ qui n'est plus que du second ordre et peut se 
représenter par $A \ldots$. Une multiplication suivie de contraction se nomme multiplication intérieure.

6" PROCÉDÉS POLR RECONNAITRE LE C.AR.ACTERE TEISORIEL. - a) Lorsqu un groupe de quantités $A(\% \ldots \%$.... déterminées par $n$ indices est tel que

(11-7) $\mathrm{A}\left(\right.$ (נ...\% \%) $\mathrm{B}_{\% \ldots} \ldots$ invariant

pour un choix arbitraire d'un tenseur B,.... à $n$ indices. dont $n$ ' covariants et $n$ 'contrevariants, on peut affirmer que $\mathrm{A}(i \% . . . \% \ldots)$ est un tenseur contrevariant d'ordre $n$ et covariant d'ordre $n^{\prime \prime}$.

Ex. : Si $\mathrm{A}(, y) \mathrm{B}^{\wedge} \mathrm{C}=$ invariant, $\mathrm{A}(,, \%)$ est un tenseur $\mathrm{A} \ldots$

ce résultat est encore exact si pour un quadrivecteur quelconque $\mathrm{B}$ le produit intérieur $\mathrm{A}(\%) \mathrm{B} \cdot \mathrm{B}=$ invariant, à condition que $\mathrm{A}(\mu, \nu)=\mathrm{A}(\nu / \nu)$.

b) Un grcupe de quantités dont le produit intéricur par un quadrivecteur arbitraire est un tenseur est lui-mêmo un tenseur.

Ex.: si $\mathrm{A}(\%, \%) \mathrm{B}$ est un quadrivecteur covariant, $\mathrm{A}(\%, \%)$ est un tenseur covariant.

$7^{\circ}$ TENSELRS FOND.MLNT.IUX. - Dans l'expression de linvariant $d s^{2}$
$(11-8)$
$d s^{2}=g_{i,} d x_{i \cdot} d x$

$d x$, est un quadrivecteur covariant arbitrairc; donc d'après

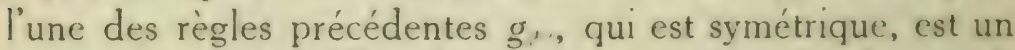
tenseur covariant. C'est le tenseur covariant fondamental. Le tenscur contrevariant fondamental g.: sobtient en écrivant le déterminant des $g_{1} .$, formant le mineur de chacun des s. . et divisant ce mineur par la valeur g du déterminant. 
D’après une propriété des déterminants on a:

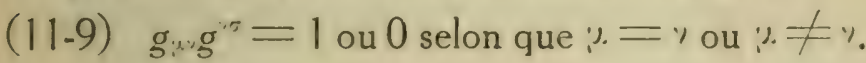

Posons $g_{\mu \tau} g^{\prime \prime \prime}=g_{j \cdot}^{\prime \prime} g_{\%}^{\prime \prime}$ est le tenseur mixte fondamental. Il jouit de la propriété d'avoir les mêmes composantes (égales à 1 pour $\mu=$ y et à 0 pour $\mu \neq \neq \nu$ ) dans tous les systèmes.

Remarquons qu'en contractant ce tenseur nous obtenons

$(11-10) \quad g_{0}^{\prime 2}=g_{1}^{1}+g_{2}^{2}+g^{3}+g_{1}^{\prime}=4$.

Remarquons aussi que g." est un opérateur de substitution, car

$$
\text { (11-11) } \quad g_{: 2}^{\prime \prime} \mathrm{A}^{\prime \prime}=\mathrm{A}^{\prime \prime}+0+0+0 \text {. }
$$

8" TENSEURS ASSOCIÉS. - Les trois tenseurs fondamentaux permettent de transformer les tenseurs par multiplication intérieure, c'est-à-dire de construire de nouveaux tenseurs en faisant passer à volonté un indice de bas en haut et inversement. Les trois tenseurs contrevariant, mixte et covariant

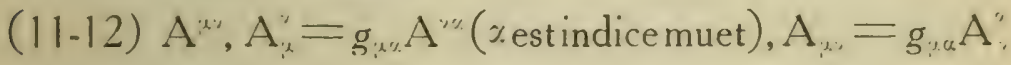
sont dits tenseurs associés.

Tout tenseur d'ordre pair permet de former un invariant appelé invariant contracté; il suffit d'amener la moitié des indices en haut, la moitié en bas et de contracter complètement.

9" LONGUEUR GÉNÉR \LISÉE. CONDITION D'ORTHOGONALITÉ. - Dans la théorie vectorielle ordinaire (3 dimensions) le produit scalaire de deux vecteurs $\mathrm{A}$ et B est :

(11-13) $\quad \mathrm{A}_{x} \mathrm{~B}_{x}+\mathrm{A}_{y} \mathrm{~B}_{y}+\mathrm{A}_{z} \mathrm{~B}_{z}=\mathrm{A}_{3} \mathrm{~B}_{4}$ 
Le carré de la longueur d'un vecteur est le produit scalaire du vecteur par lui-même. Deux vecteurs sont orthogonaux si $A_{i} B_{i}=0$.

Pour les quadrivecteurs, en coordonnées arbitraires, le scalaire

$$
\text { (11-14) } A_{B} B=A^{*} B_{2}=g^{\prime} A B=g, A B
$$

est la généralisation du produit scalaire (11-13).

Le carré de la longueur généraliscé d’un quadrivecteur $\mathrm{A}^{\prime}\left(\right.$ ou $\left.\mathrm{A}_{2}\right)$ est le scalaire

$$
(11-15) l^{2}=\mathrm{A} \cdot \mathrm{A}=g \cdot \mathrm{A}^{2} \mathrm{~A}=g \mathrm{~A} \cdot \mathrm{A} \text {. }
$$

et la condition d'orthogonalité de deux quadrivecteurs $\left(A_{: 2}, B_{i:}\right.$ ou $\left.A^{: 2}, B^{\prime 2}\right)$ est
(11-16) $\quad \mathrm{A}_{2} \mathrm{~B}^{\prime 2}=0$
ou
$\mathrm{A}^{2} \mathrm{~B}=0$.

10" DENSITÉ TENSORIELLE. - Le déterminant g des g.. est toujours $<0$; considérons $1-g$; on démontre que :

$(11-17) \backslash-g d(1)=$ invariant $\left(d(1)=d x_{1} d x_{2} d x d x\right)$.

En coordonnées galiléennes, $g_{11}=g_{22}=g_{::}=-1$. $g:=+1, \quad g_{n}=0(\because \neq y) ; 1-g=1$ et linvariant $1-g d(\omega)=d x \cdot d y \cdot d z . c d t$ (élément d'hypervolume, voir note 7).

Soit $T^{2 \ldots . . .}$ un tenseur, on appelle densite tensoriclle l'expression
(11-18)$$
\tau^{a+1}=1-g T_{1 \%}^{2 \beta \ldots}
$$

On peut toujours choisir les coordonnées de façon qu'en tout point-événement $\mid g=1$. Ce choix simplific souvent les calculs. 
$11^{\circ}$ SYMBOLES DE CHRISTOFFEL. - Nous ferons usage des symboles



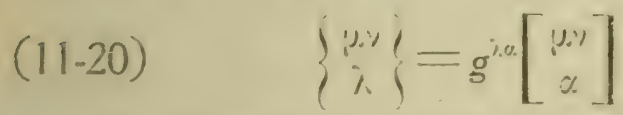

(sommation par rapport à lindice muet \%).

Les grandeurs représentées par les symboles sont nulles en coordonnées galiléennes (les g... sont constants). Ces symboles sont symétriques en '\% et \%. Il faut noter que ce ne sont pas des tenseurs.

$12^{\circ}$ DÉRIVÉE COVARIANTE. - La dérivée d'un scalaire est un quadrivecteur covariant, mais la dérivée d'un quadrivecteur n'est pas un tenseur. Soit un quadrivecteur

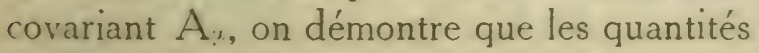

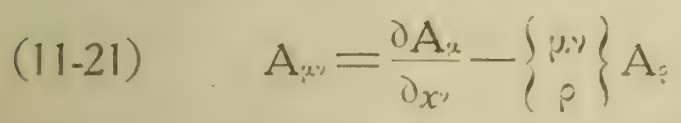

constituent un tenseur covariant, appelé dérivée covariante de $\mathrm{A}_{\ldots}$.

De même

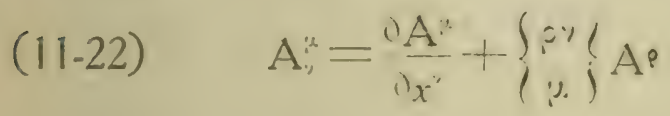

est la dérivée covariante du quadrivecteur $\mathrm{A}$. contrevariant.

Généralisation. - Soit un tenseur quelconque, A par exemple; sa dérivée covariante est le tenseur: 


\section{$(11-23)$}

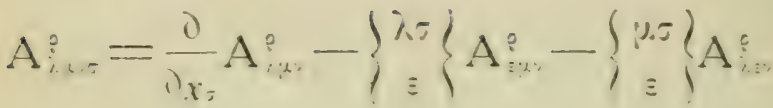



La dérivée covariante remplace, dans les équations tensorielles exigées par le principe de la relativité, la dérivée ordinaire qui en est la forme dégénércé. en coordonnées galiléennes (car en coordonnées galiléennes les symboles de Christoffel sont nuls).

Supposons qu'on déplace un vecteur suivant un certain contour. Dans un espace euclidien et en coordonnées galiléennes, la condition nécessaire et suffisante pour que le vecteur reste de même longueur et parallèle à lui-même

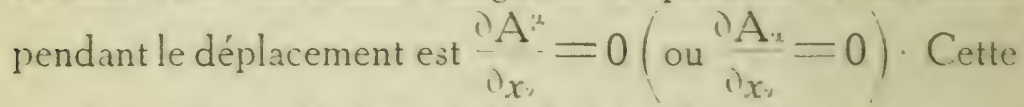
condition étant la forme dégénérée de léquation tensorielle $\mathrm{A}=0$ (ou $\mathrm{A}_{\ldots}=0$ ) nous dirons que l'annulation de la dérivée covariante d'un quadrivecteur en tout point d'un contour exprime un " déplacement sans variation absolue " (Eddington) ou un " déplacement parallèle " (Weyl) bien quil ne puisse, en général, être question de parallélisme au sens de la géométrie euclidienne.

$13^{\prime \prime}$ QLFLQUES FORIIULES UTILES. - On démontre que

$$
\begin{aligned}
& \text { (11-24) }
\end{aligned}
$$

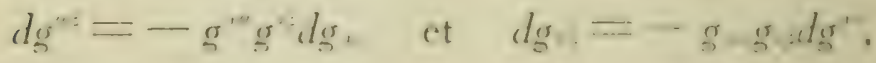

$$
\begin{aligned}
& \text { (11-25) } \quad \mathrm{A}^{\prime \prime} \mathrm{dg} \mathrm{g}_{\alpha}=-\mathrm{A}_{\alpha: \mathrm{dg}^{\alpha 3}} \text {. }
\end{aligned}
$$


$(11-26) \quad d(\log g)=-g d^{\prime \prime}=g^{\prime \prime} d g \ldots$

$(11-27)$

$$
\left\{\begin{array}{c}
\left(\mu_{c}\right. \\
i
\end{array}\right\}=\frac{\partial \log \sqrt{-g}}{\lambda_{x}}
$$

14" DIVERGE.NCE. - Dans la théorie vectorielle ordinaire, on appelle divergence le scalaire

$$
\text { (11-28) } \frac{\partial \mathrm{A}_{x}}{\partial x}+\frac{\partial \mathrm{A}_{y}}{\partial y}+\frac{\partial \mathrm{A}_{z}}{\partial z} \text { ou } \frac{\partial \mathrm{A}}{\partial x_{i}}
$$

La généralisation est immédiate. Pour un quadrivecteur contrevariant, la divergence est la dérivée covariante con-

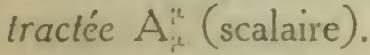

Introduisant la densité tensorielle, et tenant comptc de $(11-27)$

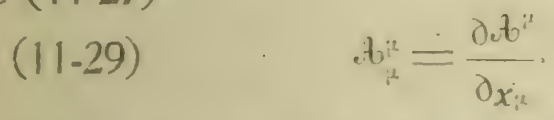

La divergence d'un tenseur du second ordre est, de même, la dérivée covariante contractée : c'est un quadrivecteur.

a) Tcnscur mixlc $\mathrm{A}_{. .}$- La divergence est $\mathrm{A}_{. .}$(, devient indice muet)

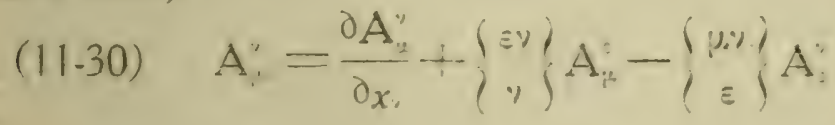

ou

$$
\text { (11.31) } \quad b_{\mu \nu}^{\prime \prime}=\frac{\partial A_{\mu}}{\partial x}-\left\{\begin{array}{c}
u . y \\
z
\end{array}\right\} b^{\prime \prime}
$$

expression qui, lorsque $A$... est symétrique, se simplific

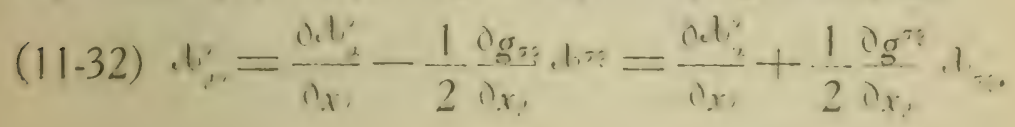


b) Tchscur contretariant A' - La divergence est A. ; en introduisant les densités tensoriclles, on trouve

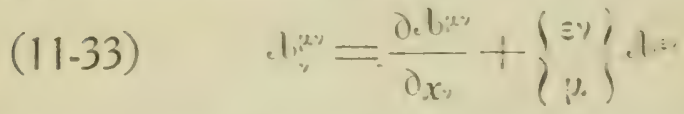

le dernier terme disparait lorsque le tenseur est symétrique gauche.

Dans la théorie vectorielle, lannulation de la dicergence d'un iecteur exprime la continuité du flux de ce iecteur. Dans la théorie de l'univers quadridimensionnel, où intervient une coordonnée de temps, l'annulation d'une divergence est la condition la plus générale de conseiiation ou de permanence d'un quadrivecteur ou d'un lenseur.

15 LE TENSEUR DE RIEM.A. CHRISTOFFLL. La dérivée covariante du tenseur $g_{\text {. }}$ est identiquement nulle. On peut cependant former un tenseur par différenciation à partir du tenseur fondamental scul.

Formons la dérivée seconde covariante $A_{1}$. d'un vecteur arbitraire $A_{\text {, }}$, puis le tenseur $A_{1}=-A_{\ldots}$, le calcul donne



$(11-34)$

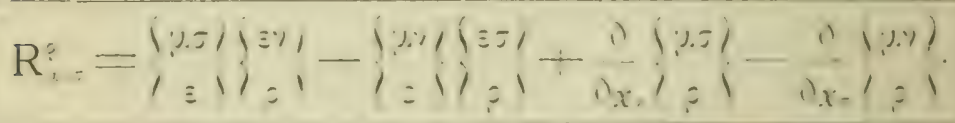

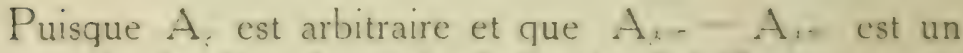
ienseur, la dernicre des règles indiquáes ( ${ }^{\circ}$ "6) montre que $\mathrm{R}$ :- est un tenseur. II niest constitue que par los g. a d leurs derivécs: c'est un tenseur d'Univers.

Le tenseur contracté est 
$(11-35)$



( $;$ et $\cong$ sont des indices muets)

qu'on peut écrire, en utilisant (11-27)

$(11-36)$



Les deux derniers termes disparaissent si l'on choisit les coordonnées de manière que $1-g=1$.

Enfin, en multipliant par $g^{\prime \prime}$ on forme l'invariant contracté
$(11-37)$
$\mathrm{R}=\mathrm{g}^{\mathrm{kn} / \mathrm{R}}$

qu'on démontre être une généralisation de la courbure de Gauss.

Note 12.

Gravitation et dynamique.

$1^{\circ}$ LOI DE LA GRAVITATION DANS LE VIDE. - Si I'Univers est euclidien, et si l'on prend des coordonnées galiléennes, toutes les composantes de $\mathrm{R}_{\ldots-}^{\prime}$ s'annulent $\mathrm{car}$ tous les symboles de Christoffel sont nuls. Mais alors ces composantes s'annulent aussi dans tout autre système de coordonnées (propriété fondamentale des tenseurs, les équations de transformation des composantes étant linéaires et homogènes). L'annulation du tenseur de RiemannChristoffel est donc une condition nécessaire pour que l'Univers soit euclidien. On démontre que cette condition est suffisante. 
Si l'on admet que l'Univers est euclidien à distance infinie de toute matière, la loi de la gravitation dans le vide est nécessairement $R_{k}=0$ (voir chap. $x$ ).

Mais si l'Univers est courbe dans son ensemble et si l'espace est fini, il n'est plus nécessaire de conserver $\mathrm{R}_{\ldots \pi}=0$ comme solution limite, et la covariance est respectée sil'on pose
$(12-1)$
$\mathrm{R}_{\mathrm{i}}^{\prime}=\mathrm{R}$
$\mathrm{R}_{\mathrm{w},}-\lambda \mathrm{g}_{\mathrm{k},}=0$

$\therefore$ étant une constante universelle certainement très petite. Pour le moment nous supposerons $\%=0$.

$R^{\prime}$. est la seule expression générale d'un tenseur du second ordre, fonction seulement des g. ct de leurs dérivées, ne contenant pas de dérivées d'ordre $>2$ et linéaire par rapport aux dérivées secondes.

2" THÉOREME FOND.MMEVTML. - L La divergence du icnseur

$$
\mathrm{R}_{;}^{\prime \prime}-\frac{1}{2} \mathrm{~g}_{i}^{\prime \prime} \mathrm{R}
$$

cst identiquement nulle, ce qu'on peut écrire
$(12-2)$

$$
\mathrm{R}_{z^{\prime},}^{\prime \prime} \equiv \frac{1}{2} \frac{\partial \mathrm{R}}{\partial x_{i, 2}}
$$

Ces quatre identités $(y=1,2,3,4)$ sont celles guu réduisent à 6 le nombre des conditions exprimant la loi do la gravitation dans le vide (Ch. X, p. 108).

3" ÉQUATIONS DES GÉODISIQUFS. - Soit A $\mathrm{A}^{\prime}$ vecteur contrevariant $\frac{d x_{\tau}}{d s}$. Sa dérivée covariante est

$$
(12-3) \quad A_{0}^{0}=\frac{i}{\partial x_{0}}\left(\frac{d x}{d s}\right)+\left\{\begin{array}{c}
\left.x_{i} j\right) d x \\
j
\end{array}\right) d s
$$


Multiplions par $\mathrm{A}^{*}=\frac{d x_{\mathrm{a}}}{d s}$ nous obtenons
$(12-4)$

$$
\mathrm{A}^{\alpha} \mathrm{A}_{\alpha}^{\sigma}=\frac{d^{2} x_{s}}{d s^{2}}+\left\{\begin{array}{c}
x_{0} \\
\tau
\end{array}\right\} \frac{d x_{\alpha}}{d s} \frac{d x_{3}}{d s} .
$$

Le premier membre étant un tenseur, il en est de même du second. Ce tenseur s’annule en coordonnées galiléennes pour tous les points d'une géodésique car alors $\frac{d^{3} x^{3}}{d s^{2}}=0$ et $\mid=1=0$; par suite l'annulation de ce tenseur représente les équations d'une géodésique dans un Univers euclidien quelles que soient les coordonnées.

D'après le principe d'équivalence, il en est de même dans un champ de gravitation permanent. L'équation générale est donc

$$
\frac{d^{2} x}{d s^{2}}+1=\left\{\frac{d x}{d s} \frac{d x}{d s}=0\right.
$$

$$
\text { (4 équations: } \sigma=1,2,3,4 \text { ). }
$$

Il est à remarquer que le principe d'équivalence n'est autre chose que l'affirmation de l'existence d'un Univers tangent. Il résulte de là qu'il y a nécessairement équivalence entre un champ de force géométrique dans un Univers euclidien et un champ de gravitation permanent pour les lois qui ne font intervenir que les $g$ et leurs dérivées premières, mais qu ill n'y a plus nécessairement équivalence pour les lois faisant intervenir les dérivées des g g. d'un ordre supérieur au premier.

$4^{\circ}$ LOI DE. L.I GRAVITATION D.ANS LA M.ATIERL: - Les équations \ $\mathrm{R}_{\mu^{v}}=0$, qui expriment la loi ldans le 
vide, remplacent l'équation de Laplace $د \Omega=0\left(\Delta=\frac{\partial^{2}}{\partial x^{2}}+\frac{\partial^{2}}{\partial x^{2}}+\frac{\partial^{2}}{\partial x^{2}}\right) \Omega$ potentiel newtonien.

Il sagit maintenant de trouver l'équation qui doit remplacer l'équation de Poisson

$\Delta \Omega=4-\mathrm{G}_{i}$ (: densité, $\mathrm{G}$ const. de la gravit. newt. $)$.

La densité est l'énergie par unité de volume divisée par $c^{2}$. Or l'impulsion-énergie trouve son expression la plus générale dans un tenseur qui précisément se réduit à : dans le cas de la matière au repos, en coordonnées galiléennes. C'est ce tenseur qui doit remplacer $\hat{i}$.

a) Le tenseur impulsion-énergie. - Les trois tenseurs d'impulsion-énergie associés ont pour expressions

$(12-6)$

$$
\mathrm{T}^{\mathrm{k},}=\rho_{0} \frac{d x_{\mathrm{z}}}{d s} \frac{d x_{\mathrm{v}}}{d s}
$$

(:" densité au repos ou densité propre)

(12-7) $\quad \mathrm{T}_{\mathrm{i}}^{\prime}=g_{\mathrm{k}, 5} \mathrm{~T}^{\sigma \nu}=g_{\mathrm{i}, \rho_{1}} \frac{d x_{\tau}}{d s} \frac{d x_{\mathrm{v}}}{d s}$



T" et $T$.. sont symétriques. En coordonnées galilécnnes $x_{i}, x_{3}, x, x_{i}=c t$, les composantes de $\mathrm{T}_{2}$, sont les suivantes.

$$
\begin{aligned}
& T_{: 2}^{\prime \prime}=-\frac{1}{c^{2}} \rho v_{x_{1}}^{2}-\frac{1}{c^{2}} \rho v_{x_{1}} v_{x}-\frac{1}{c^{2}} \rho v_{x_{1}} v_{x_{3}}-\frac{1}{c} \rho v_{x_{1}} \\
& r^{\prime \prime}-\frac{1}{c^{2}} \rho v_{x_{2}} v_{x_{1}}-\frac{1}{c^{2}} \rho v_{x_{2}}^{2}-\frac{1}{c^{2}} \rho v_{x_{2}} v_{z_{3}}-\frac{1}{c} \rho v_{x_{2}}
\end{aligned}
$$




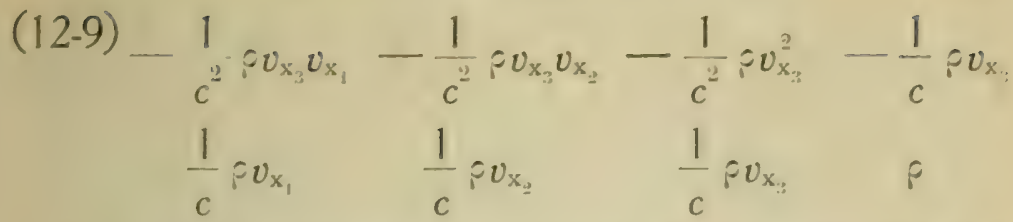

$v_{1}, i_{x}, v_{x}$, sont les composantes de la vitesse $i$ de la matière au point $\mathrm{x}_{1}, \mathrm{x}_{2} \mathrm{x}$, ${ }_{i}$ est sa densité, égale à

$$
\frac{1}{\alpha^{2}} p_{0}\left(\alpha=\sqrt{1-\frac{v^{2}}{c^{2}}}\right) \text {. }
$$

b) La loi de conservation de l'impulsion-énergie. - En coordonnées galiléennes l'expression de la loi de conservation s'écrit

$$
\text { (12-10) } \quad \frac{i T_{2}}{i_{x}}=0 \quad \text { (" indice muet). }
$$

Ces quatre équations $(\mu=1,2,3,4)$ ne sont autres que les équations bien connues de l'hydrodynamique en l'absence d'un champ de force et dans les milicux dépourvus de frottement.

Nous remarquons que l'équation (12-10) est la forme dégénérée de l'équation tensorielle

$$
\text { (12-11) } \quad \mathrm{T}_{i, \nu}^{\prime \prime}=0 \text {. }
$$

Cette équation tensorielle est donc l'expression générale de la loi, dans un Univers non euclidien. Nous avons d'ailleurs déjà dit que l'annulation de la divergence exprime la conservation.

c) La loi d'Einstein. - Du fait que les tenseurs $T_{\text {; }}$ et $R ;--\frac{1}{2}{ }_{j}{ }_{j}{ }_{2} R$ ont tous deux une divergence nulle, il ne 
résulte pas forcément que ces tenseurs sont égaux (à un facteur constant près). Cependant si, avec M. Eddington. nous posons en principe que tout tenseur physique est l'aspect sous lequel nous apparait un tenseur géométrique d'Univers, et si nous considérons la loi de conservation de l'impulsion-énergie comme une loi expérimentalement établie et rigoureuse, $T^{\prime}$ doit être identifié avec un tenseur conservatif: comme le plus simple des tenseurs conservatifs est $\mathrm{R}-\frac{1}{2} \sigma^{\circ} \mathrm{R}$, nous sommes conduits à écrire
$(12.12)$

$$
R-\frac{1}{2} g: R=-\% T_{*}^{\prime \prime}
$$

$\%$ constante universelle

quitte à vérifier ensuite par l'expérience les conséquences de cette loi.

C'est la loi d'Einstein, mais Einstein a suivi pour l'établir une marche différente. Il a mis $\mathrm{R}=0$ sous la forme des équations classiques de Lagrange, a reconnu que certaines quantités $t$. (au nombre de seize, mais en formant pas un terseur) représentent une forme d'énergie. l'énergie de gravitation, et a ajouté simplement le tenseur impulsion-énergie T à l'énergie de gravitation (il a remplacé $t$, par $t,-T$ ? Il a ainsi obtenu la loi précédente. Cette loi impose la conservation de l'impulsion-énergie car, la divergener du premier membre étant identiquement nulle, la divergencr. du second membre est nulle.

Cette loi se déduit aussi du principe daction station. naire (méthode de MM. Hilbert et Lorentz).

In loi de la gravitation peut encore se mettre anus 
d'autres formes
$(12-13)$
$\mathrm{R}_{\mu \nu}-\frac{1}{2} g_{k,} \mathrm{R}=-\% \mathrm{~T}_{k \nu}$

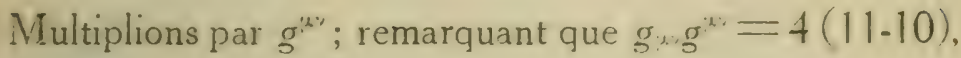
nous obtenons
(12-14)
$\mathrm{R}=\% \mathrm{~T}=\%$
$\left(\mathrm{T}=\mathrm{g}^{\ldots} \mathrm{T} \ldots\right)$

car on voit facilement, d'après (12-9), que

$$
\begin{aligned}
& \text { (12-15) }
\end{aligned}
$$



valeur indépendante du système de coordonnées, puisque c'est un scalaire.

Remplaçant, dans (12-13), R par \%T, la loi s’écrit

$$
\text { (12-16) } \mathrm{R}_{\ldots}=-\%\left(\mathrm{~T} \ldots-\frac{1}{2} g_{\mathrm{N}} \mathrm{T}\right) \text {. }
$$

5" LES ÉQUATIONS DE L'HYDRODYNAMIQUE. En mécanique classique, les quatre équations de l'hydrodynamique dans un champ de force peuvent se mettre sous la forme suivante, où,$F_{x_{1}} \ldots$ sont les composantes de la force s'exerçant sur l'unité de volume.

$$
(12-17) \frac{\partial T}{\partial_{x}}=-\left(\frac{F_{1},}{c^{2}} F_{1}, F_{x}, 0\right)
$$

en coordonnées galiléennes. En réalité il ny a plus de telles coordonnées, mais c'est un fait dont on ne tient pas compte.

Les équations rigoureuses sont les équations $\mathrm{T}:=0$, qui s'écrivent d'après (11-31) 


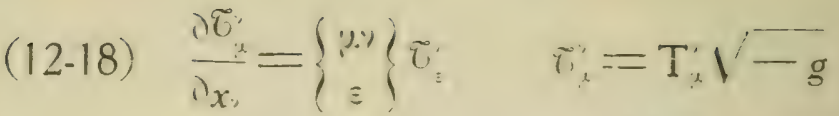

(densité tensorielle).

Ce sont les quatre conditions $(\mu=1,2,3,4)$ auxquelles la matière doit satisfaire; elles déterminent l'impulsion et l'énergie communiquées à la matière par le champ de force.

Pour les comparer aux équations anciennes, comme en pratique l'Univers est presque euclidien et que les vitesses sont faibles, nous pouvons prendre des coordonnées très voisines des coordonnées galiéennes, les choisir de manière que $1-g=1$ et admettre que $T_{\text {s }}$ se réduit à $T$ : nous obtenons l'approximation faite en mécanique ordinaire

$$
\text { (12-19) } \frac{\partial \mathrm{T}_{x}}{\partial x}=\left\{\begin{array}{c}
\cdot 4 \\
4
\end{array}\right\} \mathrm{T}_{t}=\left\{\begin{array}{l}
1.4 \\
+1
\end{array}\right.
$$

Les forces. - Comparant (12-19) et (12-17) nous

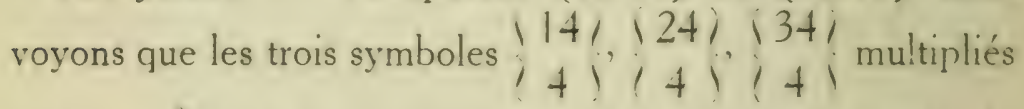
par $-c^{2}$ sont les composantes de la force principale, la force d'inertie de la mécanique qui produit une action proportionnelle à l'énergie (masse): la mécanique newtonienne néglige en général les autres "forces " liées aux autres composantes du tenseur $T_{\%}^{\prime}$ c'est-à-dire aux quantités de mouvement et aux tensions internes.

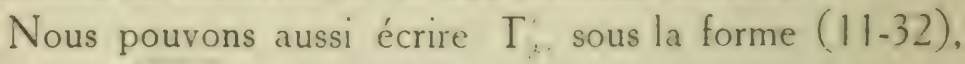
et faire $1-g=1$ nous obtenons, en première approximation

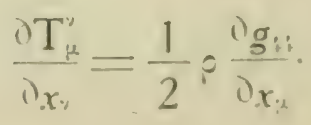


De sorte que

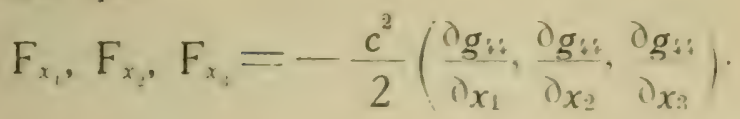

Si 12 est le potentiel au sens de la mécanique classique, on a

$$
11=c^{2} g_{i:+}+c^{t e}
$$

et si, à l’infini $\Omega=0$ et $g_{::}=1$ (valeur galiléenne)

$$
(12-21) \quad g_{i}=1+\frac{2 \Omega}{c^{2}} \text {. }
$$

6" L.I LOI DU MOUVEMENT DU POINT M.ATÉRIEL LIBRE EST CONTENUE DANS LA LOI DE LA GRAVITATION. - Si l'on adopte des coordonnées devenant galiléennes à l'infini, on trouve aisément que les équations des géodésiques se réduisent en première approximation aux équations du mouvement de la vieille mécanique, et l'on obtient en même temps (12-21), relation que nous venons de déduire de la loi de conservation, c'est-à-dire de la loi de la gravitation qui implique la conservation du tenseur $T_{2}$.

Ce résultat laisse penser qu'il n'y a pas indépendance entre la loi de la gravitation et la loi suivant laquelle un mobile libre a pour ligne d'univers une géodésique. On peut le voir de différentes manières : voici une démonstration due à M. Jacques Rossignol.

Prenons des coordonnées telles que $I_{--g}^{-g} \mathrm{I}$, on a




explicitant $T_{22}^{*}$ et $T^{\alpha .9}$, on obtient

$$
\frac{1}{d x}\left(\frac{d x}{d s} \frac{d x}{d s}\right)+\{j\} \frac{d x}{d s} \frac{d x}{d s}=0
$$

Développant le premier terme, posant $u^{\tau}=\frac{d x_{\tau}}{d s}$, et multipliant par $u_{r}$ on trouve

$$
\frac{\partial u}{d x}=0 \quad \text { ou } \quad \rho_{u} \frac{\partial u}{\partial x}=0
$$

qui $1^{\circ}$ exprime la conservation de la masse; $2^{\circ}$ réduit l'expression précédente à l'équation des géodésiques. Levant la restriction $\backslash-g=1 \frac{\partial u^{\prime \prime}}{\partial x_{v}}=0$ devient $\frac{\partial}{\partial x_{v}}(u \sqrt{-g})=0$, et l'équation des géodésiques ne change pas, car le $l^{\mathrm{m}}$ membre de cette équation est un tenseur.

On voit, par les résultats qui précèdent, que la loi d'Einstein contient toute la dynamique.

7" L.I LOI DE NEWTON. - Prenant, dans un champ staiique, des coordonnées très voisines de coordonnées galiléennes et devenant galiléennes à l'infini, négligeant toutes les quantités très petites, réduisant $T \ldots$ à $T_{0}=\beta$, confondant $T_{1}=\hat{p}$ et $T=\hat{\beta}$, tenant compte enfin de. $(12-21)$, on trouve que la formule $(12-16)$ se réduit en première approximation à l'équation de Poisson

$$
\Delta \Omega=4 \pi \mathrm{G},
$$

avec la relation
$(12-22)$
$\%=\frac{8-\mathrm{G}}{c^{2}}=1.87 \cdot 10$
unite C. G. S.

8. PROPAGATION DI LA GRAVTTITIOY. 
Dans un champ non statique, au lieu de l'équation de Poisson on obtient (après calculs compliqués)

$(12-23) \quad\left(-\frac{\partial^{2}}{\partial x_{1}^{2}}-\frac{\partial^{2}}{\partial x_{2}^{2}}-\frac{\partial^{2}}{\partial x_{1}^{2}}+\frac{\partial^{2}}{\partial x_{1}^{2}}\right) h=2 \mathrm{R}$.

Les $h$. étant les différences, supposées tres petites, entre les valcurs des g. et les valeurs constantes galiléennes. Dans le vide $R_{\ldots}=0$ et l'équation (12-23) exprime que les perturbations gravifiques se propagent avec la vitessc de la lumière $\left(\operatorname{car} x_{i}=c t\right)$.

\section{Note 13.}

LE CHAMl, de Gravitation d'Un CENTRE Matértel.

1 EXPRESSION DE $d s^{2}$, - Dans un Univers cuclidien, si l'on prend des coordonnées polaires

$$
x_{1}=r, \quad x_{2}=0, \quad x_{i}=r_{i}, \quad x_{i}=c t .
$$

l'intervalle élémentaire est

$$
d s^{2}=-d r^{2}-\left(r^{2} d f^{2}+r^{2} \sin { }^{2} y d t^{2}\right)+c^{2} d t^{2} .
$$

Dans le champ de gravitation qui règne autour d'un centre matériel, il n'y a plus de coordonnées euclidiennes, mais on peut, prenant la particule pour origine des conrdonnées, essayer de mettre $d s^{2}$ sous une forme analogue, quitte à chercher ensuite la signification des coordonnées. Nous voyons d'abord que pour que le résultat ait une signification physique, il faudra choisir des coordonnées telles que les notions d'espace et de temps soient conservées; il résulte de là qu'il ne faut pas introduire de termes en drd"), etc..., drdt, etc..., à cause de la symétrie " dans l'espace" de la particule et de son champ, et de la symé- 
trie " dans le temps" de son histoire passée et future, suivant l'expression de M. Eddington. Posons donc : $d s^{2}=-c^{2} d r^{2}-\left(r^{2} d y^{2}+r^{2} \sin ^{2}(1) \dot{r}^{2}\right)+c^{\prime} d x^{2} d x=c d t$.

On réussit effectivement à déterminer $\%$ et " (qui sont des fonctions de $r$ et non de \%, $\tau_{,} t$ et doivent s'annuler à l'infini) de manière que la loi d'Einstein $R_{-=}=0$ soit satisfaite.

On a

$$
\begin{gathered}
g_{11}=-e^{i}, \quad g_{12}=-r^{2}, \quad g \cdots=-r^{2} \sin ^{2} 1, \quad g_{i i}=e^{\prime} \\
g=-e^{i+r^{4} \sin ^{2} 0 .}
\end{gathered}
$$

Il faut écrire les équations $R_{5:}=0$ (qui se réduisent ici à $R_{11}=0, R_{2: 2}=0, R_{\cdots: 3}=0, R_{i:}=0$ ) en explicitant tous les symboles de Christoffel. On arrive, après des calculs assez pénibles, au résultat suivant (résultat rigoureux)', établi par M. Schwarzschild.

$$
\begin{array}{cc}
\text { (13-1) } d s^{2}=-\frac{1}{\eta} d r^{2}-r^{2} d t^{2}-r^{2} \sin ^{2} \theta d z^{2}+\gamma c^{2} d t^{2} \\
\text { avec } & \because=1-\frac{2 \mathrm{GM}}{c^{2} r}
\end{array}
$$

( $\mathrm{G}$ constante de la gravitation newtonienne).

1. Liobjection faite récemment par .11. Painlevé (C. R. de li.Ac de: Sel contre le: conclusion: au on peut déJuire de la formule d'Ein tein-Sciwarz-chll.t n’est pa- iu-tifiée. M1. Painlevé a employé d'autres coordonnée; et a, naturellement. trouvc une autre expression exacte de $d s^{2}$. Mais si le mathémalicien con idére à son point de vue toutes les coordonnées comme équivalentes, il n'en et pas de méme du physicien lor:que celui-ci a be:oin d'interpréter les résultat. car li choik de corlonnés peut alors se trouver impoié par la nature de grandeurs qui interviennent dans le: me-ure: expérimentales. Or le tésultat de M. I'ainleví ne saurait être interprétí physiquement parce que sa formule contient un terme en cirdt incompatible avec la symétrie dans le tenums. et que par suite les coordonnén.". empleyere nont plus de sen-au point de vue de ce que nous appelon. " distance." et "temps". Les concluions physiques de M. l'ainlevé :ont, pur cette tat. on. complètement inexactes. 
M est une constante d'intégration qu'on identifie drec la masse de la particule, car d'après
$(12-21)$
$\Omega=-\frac{\mathrm{GM}}{r}$

Cherchons maintenant la signification des coordonnées:

a) Le temps. - En un point fixe par rapport au centre matcriel $\left.\left(d r=0, d^{\prime}\right)=0, d \div=0\right)$ l'intervalle de temps mesuré entre deux événements infiniment rapprochés est

$$
d:=\frac{d s}{c}=\sqrt{\dddot{\gamma}} d t \text {. }
$$

Comme $1 \because=1$ pour $r=x$, on voit que la coordonné $t$ est le temps à distance infinie de la particule, dans un système lié à la particule.

b) L'espace. - Le terme d'espace représentant le carré de la distance de deux points infiniment voisins est

$$
\text { (13-3) } \quad d l^{2}=\frac{1}{\because} d r^{2}+r^{2} d t^{2}+r^{2} \sin ^{2} \varphi d \xi^{2} \text {. }
$$

L'espace n'est pas euclidien, cependant il s'en faut de peu car $\because$ est très voisin de 1. Transversalement $(d r=0)$ l'expression de $d l^{2}$ est la même que celle d'un arc de sphère en géométrie euclidienne ( $r$ rayon vecteur,") angle de ce rayon ayec un axe fixe, $=$ angle azimuthal). Radialement $\left.\left(d^{\prime}\right)=0, d^{\prime}=0\right)$ on a $d r=1 \% d l$. On voit que les longueurs mesurées transversalement (une circonférence, par exemple, ayant pour centre la particule) sont les mémes que si l'espace était euclidien, mais qu'il en est autrement pour les longueurs mesurées radialement (le diamètre de la circonférence), les mesures étant faites dans les deux cas arec la mème règle tries courte dl. Il résulte 
de là que le rapport de la circonférence au diamèrc est légèrement inférieur à -, mais l'écart est laible: si une masse de 1 tonne était à l'intérieur d'un cercle de 5 mètres de rayon, c'est seulement la 2f" décimale qui serait changée (M. Eddington).

Pratiquement, $r$ et $t$ sont "la distance" et "le temps". 2" MOUVEMENT DES PL. IVITES. - Supposant l. vitesse initiale dans le plan 1$)=\frac{\pi}{2}$ c'est-a-dire posanl initialement $\cos \theta=0 \mathrm{ct}-\frac{d^{(1)}}{d s}=0$, il suffit de transporier, dans l'équation générale des géodésiques, les valeurs des symboles de Christoffel trouvées dans le calcul de $d . s^{*}$, pour obtenir

a) $\frac{d^{2} \theta}{d s^{2}}=0$, qui prouve que la rajectoire reste dans le plan $6=\frac{\pi}{2}$.

b) Les équations du mouvement suivantes :

$(13-5)$

$$
\begin{aligned}
\left(\frac{d}{d s}\right)^{+}+\left(\frac{d s}{d s}\right)^{-}= & (\mathrm{K}-1) \\
& +\frac{2 \mathrm{GM}}{c^{2} r}+\frac{2 \mathrm{GMI}}{c^{2}} \frac{h^{2}}{r^{3}}
\end{aligned}
$$

$$
r \frac{d t}{d s}=h \quad(\mathrm{~K}, h \text { constantes d'intégration }) .
$$

au lieu des équations newtonienrics

$(13-6)\left\{\begin{array}{l}\left(\frac{d r}{d t}\right)+r\left(\frac{d}{d t}\right)=-\frac{G M}{a}+\frac{2 G M}{r}, \\ r^{2} d s=h \quad(1 \text { demi grand ase de l'orbite }),\end{array}\right.$ 
a part le terme supplémentaire $\frac{2 \mathrm{GM}}{c^{2}} \frac{h^{2}}{r^{3}}$. on peut identifier $(13-5)$ et $(13-6)$ en posant $\mathrm{K}^{2}=1$

a.

Le terme supplémentaire entraine un déplaccment du périhélic.

On trouve pour ce déplacement, en fraction de tour par période,

$(13-7)$

$$
\frac{3 \mathrm{GM}}{c^{2} a\left(1-\mathrm{e}^{2}\right)}
$$

(e excentricité).

3' PROP.IG.ITION DE L.I LUMIERE. - Faisant $d_{s}=0$, on obtient pour le mouvement dans le plan $=\frac{\pi}{2}$.

(13-8) $\quad \frac{1}{\because}\left(\frac{d r}{d t}\right)^{2}+\left(r \frac{d}{d t}\right)^{2}=\because c^{2}$

propagation radiale (13-9) $\frac{d r}{d t}=\because c$

propagalion transiersale (13-10) $r \frac{d t}{d t}=I^{/} \because c$.

Pour un rayon venant de l'infini et parvenu à l'infini après être passé à la distance minimum $\mathrm{R}$ du centre, on trouve pour la déviation (angle des asymptotes de la trajectoire) $\frac{4 \mathrm{GM}}{c^{2} \mathrm{R}}$

f' R.ILENTISSEMIENT DU TEYPS. - Soient deux événements infiniment voisins se produisant au mème point du champ de gravitation $\left.\left(d r=0, d^{j}\right)=0, d_{i}=0\right)$;

12. DECQULTLL 


\section{$(13-1)$ se réduit à}
$(13-11)$
$d=\frac{d s}{d t}=V \gamma d t$

dt est l'intervalle de temps mesuré, entre les deux événements considérés, par un observateur lié au centre matériel mais situé très loin, pratiquemeni en dehors du champ. D'autre part l'intervalle de temps propre entre les deux événements est $d$ - qui est < $d t$. Considérons deux horloges identiques $\mathrm{A}$ et $\mathrm{B}$ placées d'abord à cóté l'une de l'autre très loin du centre et marquant la même heure, toutes deux mesurent $t$. Transportons l'horloge, A à la distance $r$ du centre; elle va mezurer $\int d t<\int d t$; elle va donc marcher plus lentement et si on la ramène près de l'horloge B, elle aura pris du retard sur cette dernière.

Déplacement des raies spectrales. - Soit z̀s l'intervalle, indépendant du champ de gravitation, entre deux phases égales de l'émission. L'observateur terrestre, qui est en un point où le champ est négligeable, mesure





si la sourcé est sur le soleil. Mais si la même source eat sur la terre, il mesure $:-$. Or $\vdots t>3-$ done les raies du spectre solaire (ct des spectres stellaires) doivent étre légèrement déplacées vers le rouge.

Note 14

LES LOIS GÉNÉRALES de L'Électromagnétisme.

I" GÉNÉRALIS.ITION DES ÉQUATIONS DE MNX. WELL. - Dans la thérie ordinaine (Univers eudidien at 
coordonnćes galiléennes) on a les équations de MaxwellLorentz (note 10). Nous allons chercher la forme tensorielle générale dont elles sont une forme dégénérée.

Soient $\mathrm{G}_{1}, \mathrm{G}_{2}, \mathrm{G}_{1}$ les composantes du potenticl vecteur (unités électromagnétiques) et 'ب le potcntiel scalaire (unités électrostatiques) de la théorie habituelle. En vue de la généralisation, posons

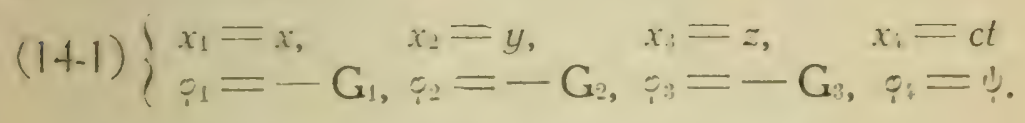

On a, avec cette notation (formules connues)

$$
\begin{aligned}
& \text { (14-2) } \mathrm{X}=\frac{\partial \xi_{1}}{\partial x_{i}}-\frac{\partial \varphi_{i}}{\partial x_{1}} \quad \mathrm{~L}=\frac{\partial \varphi_{2}}{\partial x_{3}}-\frac{\partial \varphi_{3}}{\partial x_{2}} \\
& \text { etc... }
\end{aligned}
$$

Ecrivons maintenant les équations de Maxwell-Lorentz (note 10) en désignant par $u, v, w$ les composantes de la densité de courant (unités électromagnétiques) et $\mathrm{P}$ la densité de charge (unités électrostatiques), (l'unité de charge étant choisie de façon que le facteur $4 \pi$ disparaisse). Nous avons

$$
(14-3) \cdot\left\{\begin{array}{l}
\frac{\partial L}{\partial x_{i}}+\frac{\partial Z}{\partial x_{2}}-\frac{\partial Y}{\partial x_{3}}=0 \\
\frac{\partial M}{\partial x_{i}}+\frac{\partial X}{\partial x_{3}}-\frac{\partial Z}{\partial x_{1}}=0 \\
\frac{\partial N}{\partial x_{i}}+\frac{\partial Y}{\partial x_{1}}-\frac{\partial X}{\partial x_{2}}=0 \\
\frac{\partial L}{\partial x_{1}}+\frac{\partial M}{\partial x_{2}}+\frac{\partial N}{\partial x_{3}}=0
\end{array}\right.
$$


(14-4)

$$
\left\{\begin{aligned}
\frac{\partial \mathrm{X}}{\partial x_{2}}+\frac{\partial \mathrm{N}}{\partial x_{2}}-\frac{\partial \mathrm{M}}{\partial x_{3}} & =u \\
-\frac{\partial \mathrm{Y}}{\partial x_{4}}+\frac{\partial \mathrm{L}}{\partial x_{3}}-\frac{\partial \mathrm{N}}{\partial x_{1}} & =v \\
-\frac{\partial \mathrm{Z}}{\partial x_{3}}+\frac{\partial \mathrm{M}}{\partial x_{1}}-\frac{\partial \mathrm{L}}{\partial x_{2}} & =w \\
\frac{\partial \mathrm{X}}{\partial x_{1}}+\frac{\partial \mathrm{Y}}{\partial x_{2}}+\frac{\partial \mathrm{Z}}{\partial x_{3}} & =\mathrm{P}
\end{aligned}\right.
$$

Soit maintenant un quadrivecteur covariant :- (arbitraire pour le moment) nous pouvons former sa dérivée covariante Sip, la différence



cst un tuncur symétrique gauche, et d'après sa formation, nous avons les identités :

$$
\text { (14-6) } \frac{\partial F_{\nu}}{\partial x_{s}}+\frac{\partial F}{\partial x_{\nu}}+\frac{\partial F}{\partial x_{s}} \equiv 0 .
$$

Puisque $F_{\text {. }}$, étant sỵmétrique gauche, n'a que 6 composantes distinctes, au signe près, posons :

$$
\begin{array}{ll}
\mathrm{F}_{1}=-\mathrm{F}_{1}=\mathrm{X} & \mathrm{F}_{2}=-\mathrm{F}_{1}=\mathrm{L} \\
\mathrm{F}_{2:}=-\mathrm{F}_{2}=\mathrm{Y} & \mathrm{F}_{31}=-\mathrm{F}_{1:}=\mathrm{M} \\
\mathrm{F}_{\mathrm{H}}=-\mathrm{F}_{1}=\mathrm{Z} & \mathrm{F}_{1}=-\mathrm{F}_{1}=\mathrm{N}
\end{array}
$$

puis donnons a $\%, \%$ = les valeurs suivantes 


$$
\begin{aligned}
& \because, \nu, \sigma=2,3,4 ; \quad \because, \%, \sigma=3,4,1 ; \quad \because, \%=4,1,2 \text {; } \\
& \because, y=1,2,3
\end{aligned}
$$

les identités (14-6) se trouvent être précisément les formules (14-3). De plus, les composantes du champ électromagnétique sont formées à partir du potentiel vecteur (changé de signe) et du potentie! scalaire (éq. 14-2) exactement comme les composantes de $F_{\ldots}$. sont formées à partir de $\vartheta_{\mu}$ (14-5).

Nous pouvons donc interpréter le premier groupe de Maxwell : les composantes du champ électromagnétique constituent un tenseur symétrique gauche

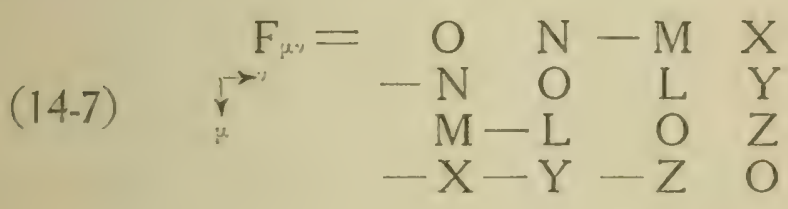

formé à partir d'un quadrivectcur potentiel $=$. dont les composantes d'espace (changées de signe) sont les composantes du potentiel vecteur et dont la composante de temps est le potentiel scalaire de la théorie ordinaire.

On peut vérifier, en transformant les composantes du tableau (14-7) suivant la loi de transformation des composantes d'un tenseur covariant, et en passant d'un système galiléen à un autre système galiléen, qu'on trouve bien les forces électrique et magnétiçuc du second système telles qu'on les obtient en relativité restreinte. Les forces électrique et magnétique constituent donc bien un tenseur.

Le tenseur contrevariant associé $F^{\prime \prime}$ permet d'exprimer le second groupe de Maxwell (14-4); ce groupe sécrit, en effet, 
$(14-8)$

ou

$$
\left\{\begin{array}{l}
\frac{\partial F^{12}}{\partial x_{2}}+\frac{\partial F^{13}}{\partial x_{3}}+\frac{\partial F^{14}}{\partial x_{1}}=u \\
\frac{\partial F^{21}}{\partial x_{1}}+\frac{\partial F^{23}}{\partial x_{2}}+\frac{\partial F^{24}}{\partial x_{1}}=v \\
\frac{\partial F^{31}}{\partial x_{1}}+\frac{\partial F^{32}}{\partial x_{2}}+\frac{\partial F^{34}}{\partial x_{i}}=u \\
\frac{\partial F^{31}}{\partial x_{1}}+\frac{\partial F^{22}}{\partial x_{2}}+\frac{\partial F^{33}}{\partial x_{2}}=I
\end{array}\right.
$$

$$
\frac{\partial \mathrm{F}^{2, v}}{\partial x}=u, v, w, \mathrm{P}
$$

ce qui prouve que $u, v, u, \Gamma$ sont les composantes d'un quadrivecteur contrevariant $J$ ' car $\frac{F^{\prime}}{i x}$ est la forme dégénérée de la divergence $F^{k}$ qui est un quadrivecteur contrevariant.

Le quadrivecteur J' est le courant. Ses composantes d'espace constituent le courant de convection et sa comprosante de temps est la densité de charce.

En résumé les équations de Maxwell s'ćcrivent

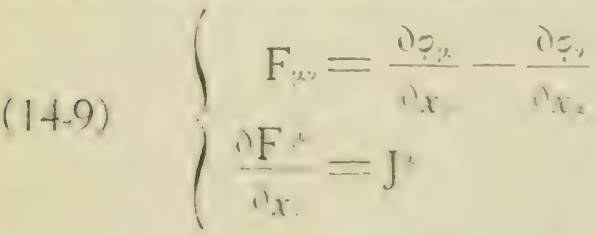

La première équation est sous la forme requise par le principe de relativité; la seconde est la forme dégénéré. de $F=J^{*}$. Les équations générales valables dans un Univers euclidien en coordonnées arbitraires, valables aussi 
dans un Univers non cuclidien par application du principe d'équivalence, sont



2' LOI DE LA CONSERVATION DE L'ÉLECTRICITÉ. - La dernière de ces équations sécrit d’après (11-33), F '́tant symétrique gauche.

$$
T_{*}=\frac{\partial T^{\prime}}{\partial x}=\gamma^{*}
$$

d'où l'on tire

$$
\frac{\partial j}{\partial x_{i}}=\frac{v^{n-i}}{\partial_{x_{i}} \partial x}=0
$$


en conrdonnées galiléennes, cette équation devient

$$
\text { (14-11) } \frac{\partial u}{\partial x}+\frac{\partial v}{\partial y}+\frac{\partial w}{\partial z}+\frac{1}{c} \frac{\partial P}{\partial t}=0
$$

semblable à l'équation de continuité de l'hydrodynamique. elle exprime la conservation de l'électricité.

3" LE TENSELR D'ÉNERGIE ÉLECTROM.AGVÉTIQUE ET LA LOI GÉVERALE DE CONSERVATION DF LIMPLLSION-ÉVERGIE. - Par application et généralisation tensorielle des expressions qui donnent les composantes de la force mécanique sexerçant sur l'unité de volume contenant charges et courants, ainsi que le travail accompli par le courant dans l'unité de temps, on démontre 
qu'il existe un tenseur d'énergie

$$
\text { (14-12) } \quad E_{\alpha}^{\prime \prime}=\frac{1}{c^{2}}\left(-F_{k \alpha} F^{\vee \alpha}+\frac{1}{4} g^{\prime \prime} F_{\alpha,} F^{\alpha}\right)
$$

dont la variation compense la variation du tenseur matériel

$$
\text { (14-13) } \quad \mathrm{E}_{\mathrm{\alpha},}^{\prime \prime}-1 \mathrm{~T}_{\alpha, \nu}^{\prime \prime}=0
$$

ce qui exprime la loi générale de conservation de l'impulsion-énergie. Dans l'expression de la loi de la gravitation, $E_{i k}^{\prime}$ s'ajoute à $T_{\%}^{\prime \prime}$.

Mais l'énergie électromagnétique ne modifie pas la courbure totale $\mathrm{R}$ car l'invariant contracté $E=E$ est nul. La courbure $\mathrm{R}$ est toujours égale à $x T=x_{1} \ldots C^{\prime}$ 'est là un fait capital qui montre que la matière ne peut pas être formée uniquement à partir du tenseur $E$, ce tenseur ne contribuant pas à la constitution de la densité matérielle.

\section{Note 15.}

LA COURBure de L'ESPACE ET DU TEMPS.

\section{I" LA COURBURE NON NULLE D.ANS LE VIDE.}

- C'est précisément le fait que l'énergie électromagnétique n influe pas sur la courbure tolalc qui nécessite une modification de la loi de gravitation admise jusqüici.

Toutes les équations où intervient la densité de la matière sont des équations macroscopiques car la matière est supposée continue. Si nous voulons écrire les équations microscopiques, nous devons faire disparaitre le tenseur


la matière) et ne conserver que le tenseur $E_{\text {; }}$ qui sera 


\section{RELATIVITÉ GÉNÉRALISÉE}

alors le tenseur exprimant l'énergie du champ des électrons. D'après la loi (12-12) la formule microscopique serait, en tout point

$$
\text { (15-1) } \quad \mathrm{R}^{\prime}-\frac{1}{2} g^{\prime \prime} \mathrm{R}=-\% \mathrm{E}_{\mathrm{\prime}}^{\prime}
$$

L'invariant contracté $E$ étant nul, celui du premier membre $R *-\frac{1}{2} g^{*}, R=-R$ devrait aussi être nul, en tout point; alors, dans la matière, la valeur moyenne de $\mathrm{R}$ serait nulle elle aussi, et comme cette valeur moyenne est égale à $x=$ il n'y aurait pas de matière; résultat absurde.

Il faut donc remplacer (15-1) par une formule dans laquelle le scalaire du premier membre soit nul. On n'a pas le choix, il faut écrire

(15-2)

$$
\mathrm{R}_{\mathrm{i},}^{\prime \prime}-\frac{1}{4} g_{;}^{\prime \prime} \mathrm{R}=-\% \mathrm{E}_{\mathrm{\prime},}^{\prime \prime}
$$

cette ćquation exprime la loi de la gravitation, $E_{\text {i }}$ étant le tenseur d'énergie du champ électromagnétique des électrons.

Sil'on forme la divergence des deux membres de (15-2), on trouve la relation

$$
\frac{1}{4} \frac{\mathrm{R}}{\partial x_{2}}=-\frac{\%}{c^{2}} \mathrm{~F}_{\cdots} \mathrm{J}^{\circ} .
$$

Partout où $\mathrm{J}^{\prime \prime}=0$ c'est-à-dire en dehors des lignes d'Univers des électrons, la courbure totale est constante; cette courbure est donc la même dans le vide et aux points où se trouic de l'énergie libre (énergie rayonnante.) Mais la courbure dans le vide n'est pas nulle car $\mathrm{R}=0$ dans le vide, ou $E^{\prime}=0$, entrainerait $R=0($ ou $R=0)$ 
et par suite la loi (12-12) seule compatible avec $R$. = 0 dans le vide; on retomberait sur la loi qu'il faut précisément modifier.

Daprès (15-2) la loi dans le vide sécrit

$$
\mathrm{R}_{\mu,}-\frac{1}{4} g_{\mu \nu} \mathrm{R}=0
$$

ou en appelant $R$. la courbure dans le vide et posant $\mathrm{R}_{0}=4 \%$
$(15-4)$

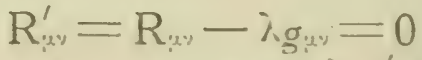

avec $i \neq 0$ mais très petit,

loi déjà indiquée note 12 .

La loi macroscopique de la matière considérće comme continue s'obtient immédiatement en remplaçant dans touic la théorie précédemment donn'e $R$, par $R$, et $R$ par $R^{\prime}=R-4 i=R-R_{1}$.

La divergence de $R_{*}^{\prime \prime}-\frac{1}{2} g_{*}^{\prime \prime} R^{\prime}$ est identiquement nulle, et ce tenseur doit étre identifié avec $T_{n}$ pour satisfaire la loi de conservation. Ia loi de gravitation dans la maticie devient

$$
\text { (15-5) } R^{\prime}=R-i \cdot g=-\%\left(T-\frac{1}{2} \pi \cdot T\right)
$$

(- la densité (au repos) est ${ }_{\%}^{1}(R \cdot R)\left(\right.$ aulieude $\left.\frac{1}{\%} R\right)$.

2" LESPACE FERME. - Cherchons mainten ani qurl peut être l'aspect ultra-macroscopique ou cosmigute de l'Univers, en accord avec la loi (15-5). Prenant comme unité de volume un espace suffisamment grand (par ex. : 
1000 parsecs-cubes), soit $\hat{i}$ la densité moyenne de la matière, densité que nous supposerons constante. Nous pouvons, dans cet aspect d'ensemble, ne tenir compte que de la distribution générale de la matière, et faire abstraction des irrégularités locales.

Les vitesses relatives des astres étant toujours très petites par rapport à la vitesse de la lumière, nous pouvons envisager un système de référence dans lequel la matière est en moyenne, au repos. T... se réduit sensiblement à

$$
\mathrm{T}_{4 i}=g_{i+i} \text {. }
$$

Les équations (15-5) s'écrivent:

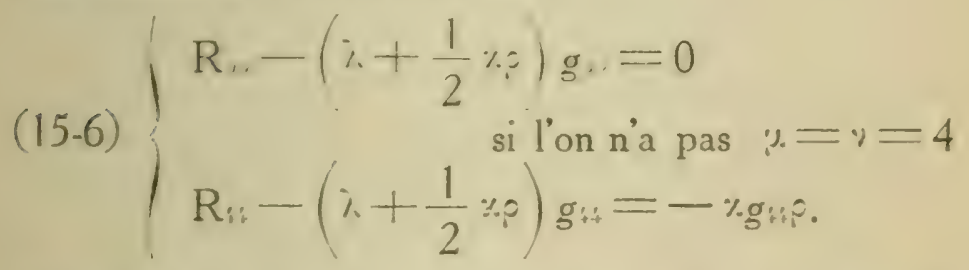

Prenant la position de l'observateur comme origine des coordonnées et adoptant des coordonnées sphériques, ces équations comportent deux solutions, dans chacune desquelles la coupe à temps constant est un espace à courbure constante positive.

$3{ }^{\circ}$ LUNIVERS D'EINSTEIN. - Soit U le raynn de courbure. La solution d'Einstein est :

$(15-7)$

$$
d s^{2}=-U^{2}\left|d t^{2}+\sin ^{2} \%\left(d t^{2}+\sin ^{2} h d t^{2}\right)\right|+c^{2} d t \text {. }
$$

avec

$(15-8) \quad \%=2 \% \quad i=\frac{1}{\mathrm{U}^{2}}$. 
$t$ est un temps d'Univers absolu. L'espace et le temps sont séparés.

Le terme d'espace est

$$
d l^{2}=U^{-}\left[d \gamma^{2}+\sin ^{2} \%\left(d t^{2}+\sin ^{2}\left(\rho d t^{2}\right)\right]\right.
$$

extension, arec une dimension de plus (coordonnée - ) de l'élément de ligne sur la surface d'une sphère ordinaire (fig. 17).

$$
d l^{2}=\mathrm{U}^{2}\left[d \%^{2}+\sin ^{2} \% d \sigma^{2}\right\} \quad(0 \text { angle azimuthal). }
$$

L'espace à courbure constante positive a deux formes possibles, l'espace sphé-

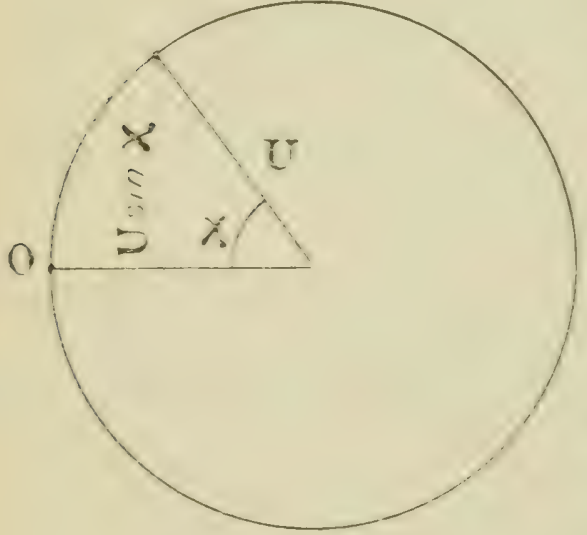

FIG. 17 rique de Riemann et l'espace elliptique de Newcomb. Adoptant l'hypothèse de l'espace sphérique, dont le volume total est $2{ }^{\prime 2} \mathrm{U}^{\prime \prime}$, la masse $\mathrm{M}$ totale de la matière mondiale serait $M=2 \pi \cdot U^{3} s$ d'où l'on déduit, d'après (15-8)

$(15-9) \quad U=\frac{\%}{4 \pi^{2}} \mathrm{M}$.

Le rayon d'Univers serait déterminé par la quantité totale de matiere. Comme ce rayon n'est sans doute pas inférieur à $10^{-1} \mathrm{~cm}$., ce résultat nécessite l'existence de quantités de matière considérablement supérieures ì celles que nous connaissons. 
4" LUNIVERS DE DE SITTER. - La sconde srolution de (15.6) est

$(15-10)$

$\left.d s^{2}=-\mathrm{U}^{2} \mid d \%^{2}+\sin ^{2} \%(d)^{2}+\sin ^{2} h l^{2}\right) \mid\left[-c^{2} \cos ^{2} \% h^{2}\right.$.

avec

$$
\text { (15-11) } \quad \hat{p}=0 \quad i=\frac{3}{\mathrm{U}^{2}} .
$$

Cet Univers est profondément différent de celui d'Einstein. g.: étant égal à $\cos ^{2} \%$ (au lieu de la constante 1) l'espace et le temps restent unis, et il y a une courbure du temps.

De plus $:=0$ montre que la courbure d'ensemble de l'Univers n'est pas conditionnée par la matière mondiale (pas plus que le rayon de la terre ne dépend des accidents du sol). La matière intervient seulement pour produire des perturbations locales que nous négligeons ici, n'envisageant que la forme d'ensemble.

La zone du temps stationnaire. - Pour un point fixe dans l'espace (par rapport à l'observateur dont la position est prise pour origine des coordonnées), on a

$$
d \%=0, \quad d^{\prime}=0, \quad d_{?}=0
$$

et

$(15-12) \cdot d s=\cos \%$ cdt ou $d t=\frac{d \%}{\cos \%}$.

Près de l'observateur $\%=0$ et $d t=d t ; t$ est le tcmps de l'observateur. Mais loin de lui, l'élément de temus propre est $d t=\frac{d s}{c}$ alors que l'élément de temps de l'olservateur est toujours dt. Dans la zone $r=\frac{1}{2}-U\left(\%=\frac{\pi}{2}\right)$ le temps 
est stationnaire pour l'obseriateur car $d t$ est infiniment grand par rapport à $d s$.

\section{Note 16.}

Généralisations de Weyl et d’Eddington.

1" THÉORIE DE WEYL. - Dans la théorie d'Einstein, l'électricité n'est pas rattachée à une proprièté géomélrique de la structure d'Univers, qui est entièrement représentée par les dix potentiels de gravitation $g .$.

M. H. Weyl a uni, dans une même géométrie, le champ de gravitation et le champ électromagnétique.

Le développement progressif de la théorie de la relativité a consisté dans la suppression des axiomes et des restrictions non nécessaires. Or, jusquà présent, il subsiste une hypothèse arbitraire : nous arons admis qu'on peut toujours, en des points d'Univers différents, employer la méme unité de mesure pour la comparaison des intervalles. A premiere vue cela parait évident : en un point d'Univers $A$, nous défnissons une unité de longueur en choisissant une règle étalon, et cette règle sert aussi pour la mesure optique du temps si nous prenons comme unité naturelle la vitesse de la lumière; il semble donc qu'en transportant en un autre point $B$ une copie exacte de l'étaIon choisi en $A$, on puisse, en $B$, mesurer les intervalles élémentaires et faire la comparaison avec les intervalles mesurés en A. Sans doute, nous pouvons opérer de la sorte si deux copies exactes de l'étalon transportées de A en $B$ par des chemins différents sont toujours identiques en $\mathrm{B}$. Or, rien ne prouve à priori, quil en soit ainsi, et si la longueur n'est pas intégrable, nous ne pouvons pas obtenir sans ambiguïté en $B$ une longueur que nous puissions 
considérer, par définition, comme représentant la même unité qu'en $\mathrm{A}$.

L'intégrabilité de la longueur (généralisée : voir note 11. $n^{\prime \prime}$ 13) est la restriction qui subsiste et qu'il faut supprimer.

Le champ de gravitation correspond à la non-intégrabilité de la direction. Soit en effet $\mathrm{A}^{*}$ un quadrivecteur; faisons-lui décrire un circuit fermé par " déplacement parallèle " (note 11, n" 16) c'est-à-dire tel qque la dérivée covariante $\mathrm{A}$; soit constamment nulle.

(16-1) $\quad \frac{\partial \mathrm{A}^{*}}{\partial x_{0}}+\left\{\begin{array}{c}y_{0} \\ \mu_{0}\end{array}\right\} \mathrm{A}^{\alpha}=0$.

La variation de ce vecteur est

$$
\partial \mathrm{A}=\int \frac{\mathrm{A}^{2}}{\lambda_{x}} d x=-\iint_{0}^{\mu}\left(\mathrm{A}^{\prime} d x\right.
$$

Posons $d \mathrm{~S}^{*}=-d \mathrm{~S}^{n}=d x_{i} d x_{s} ; d \mathrm{~S}$ est un tenseur symétrique gauche qui fait correspondre à l'aire élémentaire une direction positive de parcours sur le contour qui la limite. L'équation précédente s’écrit



$$
\begin{aligned}
& (16-2) \\
& \mathrm{A}=\frac{1}{2} \int \mathrm{R} A d S \\
& \text { de même } \quad \mathrm{A}_{\mathrm{A}}=\frac{1}{2} \iint \mathrm{R} \cdot \mathrm{A} d \mathrm{~S}=
\end{aligned}
$$

La condition nécessaire et suffisante pour que la variation soit nulle est que le tenseur de Riemann-Christoffel soit nul, c'est-à-dire que l'Univers soit euclidien. La non- 
intésrabilité de la direction caractérise donc le champ de gravitation.

De mêne, la non-intégrabilité de la longueur doil carar tériser un champ d'une autre nature. Ne serait-ce pas le champ électromagnétique?

Puisque nous ne sommes pas certains qu'on puisse défnir une unité valable en tous les points, nous devons définir une unit é en chaque point-événement de l'Linivers; nous appellerons jauge l'unité d'intervalle choisie en chaque point. Le systeme de jauges est arbitraire comme le système de coordonnées : il faut, dans le cas le plus géniral, diviser l'Univers en cellules par un système quelconque de coordonníes et dans chaque cellule infiniment petite adopter une jauge. Les jauges sont seulement soumises, it la condition d'être infiniment peu différentes dans deux cellules infiniment voisines, ce qui est possible car l'ambiguïté disparaît à la limite pour un déplacement infiniment petit. Lorsque les jauges étaient supposées les mêmes partout, dix mesures d'intervalles $d s$ autour d'un point permettaient de déterminer les dix g. et de décrire le champ de gravitation; maintenant 14 mesures vont être nécessaires pour déterminer les $g$. et + " potentiels" supplémentaircs qui paraissent bien correspondre aux composantes du quadrivecteur potentiel électromagnétique. L.es 14 potentiels 3.. et ₹ définissent la géométrie du système de coordonnées et du systéme de jauges, et contiennent en cux la structure de l'Univers.

Faisons décrire à un vecteur A, par déplurment parailiele, un contnur ferm: infiniment petit, limitant $1 S^{*}$; d'après (16-1) sa variation est
(16-3)
$d A=-\frac{1}{2}$
$R \ldots A$. A
$R, A \cdot S^{-}$ 
dA $\mathrm{A}_{*}$ est orthogonal à $\mathrm{A}_{3}$, parce que, $\mathrm{R} \ldots$ étant symétrique gauche en $\%$. et $P$, on a

$$
\text { (16-4) } \quad A^{\prime} d A=\frac{1}{2} R \cdot A \cdot A S=0 .
$$

La longucur généralisée du vecteur n'a pas changé, seule sa direction a varié. C'est la restriction admise dans la théorie d'Einstein. Supprimant cette restriction, nous devons remplacer $\mathrm{R} \ldots$ par un tenseur d'un type plus général

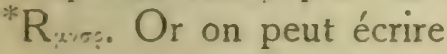

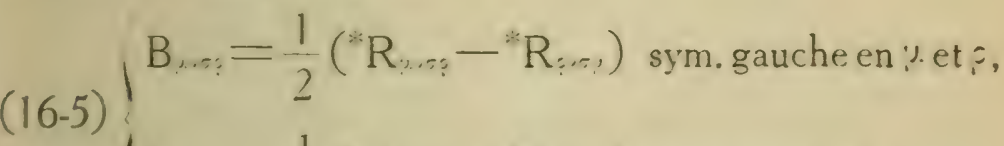

$$
\begin{aligned}
& \text { I } F=\frac{1}{2}\left({ }^{*} R_{m}+{ }^{*} R\right) \text { sym. en \%. et } \div \text {. }
\end{aligned}
$$

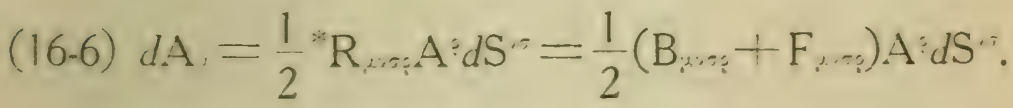

Comme la variation doit être annulée quand on décrit le circuit une seconde fois en sens inverse du premier parcours, tous ces tenseurs doivent être symétriques gauches en $y$ et $\sigma$.

Soit $l$ la longueur généralisée de $A$, ; on voit que $(l+d l)^{2}=\left(\mathrm{A}_{2}+d \mathrm{~A}^{2}\right)\left(\mathrm{A}^{2}+d \mathrm{~A}^{2}\right)=l^{2}+2 \mathrm{~A}^{2} d \mathrm{~A}$.

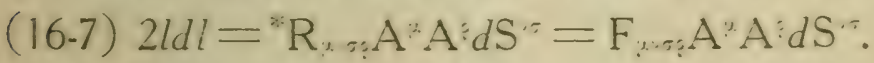

M. Weyl a adopté une limitation : il a supposé que $F$ est décomposable en $g, F_{n} ; 2^{\prime \prime}$ que $F_{n}$ est le rotationnel d'un vecteur. D'après la première condition, (16-7) devient




dl est donc proportionnet à $l$ et indépendant de la direction du vecteur.

Les différentes surfaces limitées à un même contour devant conduire à une même valeur de ¿A. l'intégrale de surface doit porter sur un rotationnel, d'où la seconde condition de Weyl.

Soit maintenant une règle extrêmement courte, de longueur généralisée l (note 11). Déplaçons-la de $d x, d x$, $d x, d x$. Fo étant le rotationnel d'un vecteur, nous pouvons écrire

$$
\text { (16-9) } \frac{d l}{l}=s_{1} d x_{1}+s_{2} d x_{2}+\tau_{1} d x+5 d x_{1}
$$

les "sétant quatre fonctions de point, qui sont les composantes d'un quadrivecteur d'Univers.

Commeles g.... les $\%$ dépendent d'une propriété intrinsèque de l'espace-temps et du systìme employé. De même que les $g_{2}$, ne peuvent pas prendre des valeurs complètement indépendantes (loi de la gravitation), de même les i. doivent satisfaire une loi.

Intégrons (16-9), nous avons

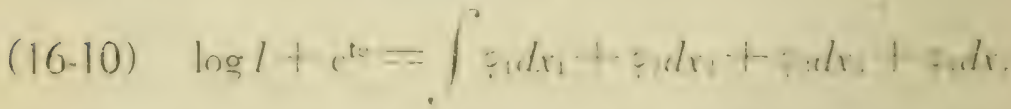

la loneueur sera indépendante du themin suisi (intesrable) si le whtionnel des : s est nul (condition dintigrabilité)

$$
(16-11) \quad \frac{i z}{n_{x}}-\frac{11}{n_{x}}=0
$$

Faisons l'hypothèe que les \& représentent le potenticl Clectromagnétigue (à un facteur consant près): lannulation 
du rotationnel exprime, d'après $(14-10)$, que le champ Clectromagnétique est nul. Si cette condition est réaliscée. les s.. suffisent pour déterminer la structure de l"Univers. Dans le cas contraire la strurture est exprimée par 14 potentiels, les g. . qui décrivent les propriétés gravifiques, les qui décrivent les propriétés électromagnétiques.

La loi des :- est trourcé : cest la généralisation tensnric:lle des équations de Maxwell. Liunion de cette lri et de celle de la gravitation constitue la loi générale de la strurture d'Univers.

Changer de système de jauges, c'est ajouter au second membre de (16-10) une fonction de point arbitraire, ou ajouter au second membre de (16-9) une différentielle tntale. Les ?. n- sont donc déterminés quà des fonctions :- près, pourvu que ces fonctions soient telles que $: d x$, soit une. différentielle exacte. Cette indétermination du systeme de jaugres ne modifie en rien le rotationnel de sorte que les forces éleclriques el magnétiques sont indépendantes du système de jauges.

Nous arons vu que la quadruple indétermination des ronrdonnćes conduit à quatre identités qui ont pour cons:quence la conservation de limpulsion-énergie. De mème. lindétermination du système de jauges entraine une loi supplímentaire de conservation : cest la conservation de l'électricité (note 14, n"2).

2 GENERILISATION DEDDINGTON, - La limitation de Weyl a pour but de donner un caractere absolu à l. longueur nulle, de manière que la lumière ait une trajes. toire hien définie (intervalle constamment nul).

Cependant M. Eddington a réussi à supprimer cetle dernière restriction. Dans la théorie d'Eddington. la variation d'un vecteur par déplacement parallìle dépend non 
seulement du chemin suivi, mais de l'orientation du vecteur pendant son déplacement. L'Univers n'est assujetti qu ì une condition : celle de posséder une structure géonétrique; c'est le moins qu'on puisse supposer, et l'on ne saurait s'élever à un plus haut degré de généralisation.

Théoric géométrique. - Prendre au système de coordonnées signifie choisir + familles d'espaces pour diviser l'Univers en cellules; dans chacune de ces familles, chaque espace peut être caractérisé par un nombre. Un déplacement $d x_{i^{-}}$est donc un iecteur absolu, puisqu'il peut s'exprimer par des nombres purs, indépendants de tout système de jauges.

M. Eddington a montré qu'en supprimant toute restriction, et conservant seulement la condition (évidemment nécessaire) que l'Univers ait une structure géométrique, et possède en chaque point un Univers euclidien tangent, la formule (16-2) est remplacée par

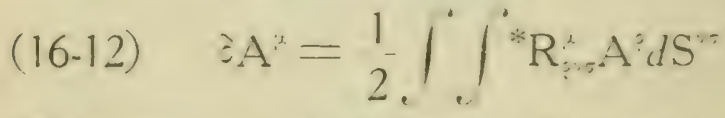


absolu, c'est-à-dire indépendant de tout sjstème de jauge's. Dans ce tenseur, les symboles de Christoffel du tenseur ordinaire sont remplacés par des symboles généralisés, qui sont absolus, parce quils sintroduisent sans que le systeme de jauges intervienne. On a

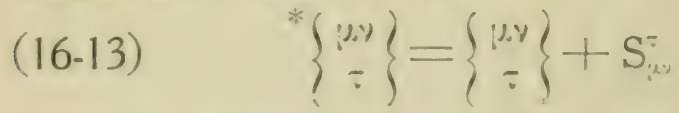

$\mathrm{S}_{\text {. }}$ étant un tenseur symétrique en : $\%$ et y (non absolu). Contractant " $\mathrm{R}_{\text {. }}^{\mathrm{*}}$, on obtient la généralisation de $\mathrm{R}$. Les deux tenseurs absolus " $R{ }^{*}$. el ${ }^{*} R$, traluisent lis 
proprefés intrinsèques du conlinuum. On n'en toil pas. d'aulres jouissunt des mémes proprićtés.

Pour introduire les $\mathrm{S}_{2}$, il faut adopter un systeme de jauges. Nous définissons la longueur / d'un déplaccment $\mathrm{A}^{\mathrm{i}}$ par

$$
\text { (16-14) } \quad l^{2}=g_{0 .} \mathrm{A}^{\prime} \mathrm{A}^{\prime} \text {. }
$$

t' est un invariant à l'égard du systeme de coordonnées ; g... est un tenseur symétrique. Un système de coordonnées étant adopté, les $\mathrm{A}$ sont des nombres purs; mais $l^{2}$ dépend, par les $g_{x}$, du systeme de jauges; la longueur n'est pas un invariant ahsolu, cest une convention purement génmétrique.
Posons
$(16-15)$$$
2 \tau=\mathrm{S}_{\sigma ; 2}^{\pi}
$$

et soit $\tau_{j}$, la dérivée covariante du quadrivecteur $=\ldots$

On peut écrire

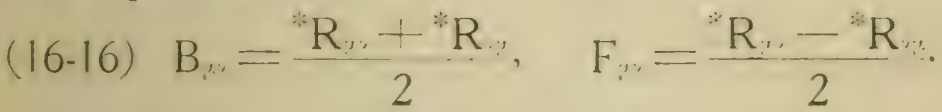
$(16-17)$

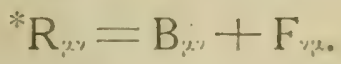

B... est symétrique et $F$ symétrique gauche. On démontre que

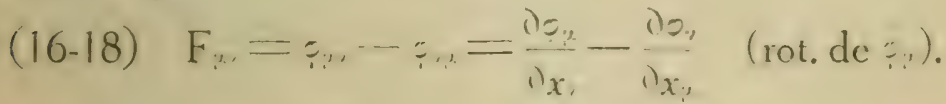

Les tenseurs $B_{\text {... et }} F_{\ldots}$... sont des tenseurs absolus.

Le tenseur $\mathrm{R}_{\ldots}$... se divise de même en deux tonseurs

(16-19)

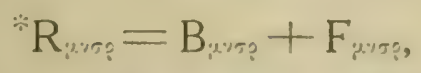

le premier symétrique gauche en is et io le second symé- 
trique en '\%. et $;$, symétriques gauches tous deux $\mathrm{cn}$ " et : Mais aucun de ces tenseurs n est absolu, car les $g, \ldots$ interviennent pour abaisser l'indice $?$.

La variation d'un vecteur est ainsi mise sous la forme (16-6) et l'on a la formule (16-7), sans aucune restriction. Iniariants absolus. - II n'existe pas de fonction invariante absolue des potentiels, mais on peut trouver des densités invariantes absolues.

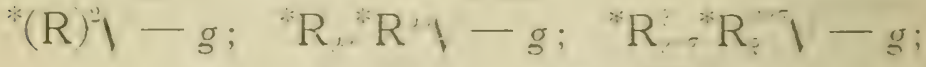

$$
\begin{aligned}
& F_{y}, F^{\prime}-g, g(R) .1-g \text {. }
\end{aligned}
$$

$\mathrm{R}$ étant le scalaire $g{ }^{*} \mathrm{R}$... Il existe peut-étre encore une densité invariante absolue dérivée de " $R$......

Le nombre des caracteres d'Univers distincts dont les combinaisons peuvent s'exprimer par des nombres purs. indépendants de tout systeme de coordonnées et de jauges ne dépasse probablement pas 6.

W eyl a fait remarquer que c'est seulement dans un Univers à nombre pair de dimensions que les tenseurs fondamentaux donnent naissance à des densités invariantes absolues. On ne saurait imaginer un unicers is nombre impair de dimensions, car il n'aurait aucun caractère absulu.

En plus de ces densités absolues, qui sont des caracteristiques absolues de l'Univers en chaque point, il y a un invariant absolu simple lié à un déplacement A' : c'est

$$
{ }^{*} R_{i \alpha}, A^{* 2} A^{*} \text {; }
$$

d'autres combinaisons plus compliquées pourraient ître imaginées.

IDENTIFIC.ITIONS PHYSIQUES. - Le systeme de jauges naturel. - Si nous voulons que la longueu. 
(form. 16-14) cesse d'étre une convention géométrigue: pour devenir une entité physique, il faut que $l^{2}$ soit un invariant absolu. Or il n'existe qu'un invariant absolu lié

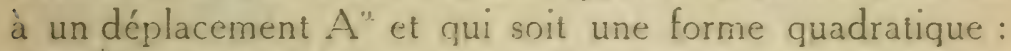
c'est ${ }^{*} \mathrm{R}$. A* A*. Nous sommes donc conduits à considérer cet invariant comme donnant une mesure naturelle de la longueur et nous devons poser

$$
\begin{aligned}
& \text { (16-20) } \quad l^{-}=\mathrm{g} \cdot \mathrm{A}=\frac{1}{i} \mathrm{R} \cdot \mathrm{A} \cdot \mathrm{A}=\frac{1}{i} \mathrm{~B}, \mathrm{~A} \cdot \mathrm{A} \text {. } \\
& \text { D’où } \\
& \text { (16-21) } \\
& B_{k, \nu}=\log _{j \%,}
\end{aligned}
$$

i. étant une constante universelle, qui nous laisse d'ailleurs libres d'adopter telle unité de longueur que nous voulons en un point d'Univers déterminé. Le choix étant fait en un point, les jauges en tous les points sont fixées par (16-21).

La différence qui sépare $B$. du tenseur $R$. de la théorie d'Einstein provient des termes issus de $S_{j}$.. Nous allons voir que ce tenseur $\mathrm{S}^{-}$. détermine les phénomènes électromagnétiques; plus le champ électromagnétique est faible, c'esta-dire plus l'espace est vide, plus $B$,., est voisin de $R$.. Dans le vide, l'équation fixant le système de jauges est

$$
\text { (16.22) } \quad R_{\mu}-7 . g_{i \cdots}=0 \text {. }
$$

C'est précissóment la loi de la gravitation d'Einstein, qui est obtenue ainsi par des considérations aussi générales que possible, absolument indépendantes de celles qui ont été exposées précédemment. Ce résultat nous montre que, dans le vide, l'Univers est effectivement jaugé conformément au systeme de jauges naturel, ou encore qu'en transportant les 
étalons d'un point à un autre pour la comparaison des intervalles on emploie, dans le vide, le systeme naturel.

Propagation de la lumierc. - Une perturbation lumi. neuse issue d'un point occupe dans l'univers un cônic qui doit satisfaire une équation de la forme
(16-23)
$a_{i x} d x_{2} d x=0$.

Comme ce cône est bien déterminé et n'a aucun rậport avec un système quelconque de coordonnées ou de jauges, il est nécessaire que $a, .$. soit un tenseur absolu : ce ne peut être que ${ }^{*} R_{\ldots \%}$. On a donc
(16-24)
R. $d x d x$
B. $d x \cdot d x=0$.

Nous royons que, dans la théorie d'Einstein, nu la jur. pagation de la lumière s'exprime par

$$
d s=g_{i, \nu} d x_{i,} d x,=0,
$$

l'Univers est jaugé conformément à l'équation (16.21), partout où la lumière se propage, c'est-à-dire en tout point (sauf à lintéricur de l'électron). is pourait ètre une fonction de point, mais la loi de la gravitation dans le vide nous montre que c'est une constante.

Le fait que, dans nos observations, la lumiere a une propagation parfaitement définie prouve que nous effectuons nos mesures avec le systeme de jauges nafurel. II est wai que dans un champ électromagnétique il existe une ambi. guïté concernant la longueur, mais cette ambiguïté disparait pour un déplacement infiniment petit, et si nous transportons nos étalons d'un point à un autre dans un domaine très petit pour comparer des intervalles, nous employons le systeme naturel à une quantité du second ordre près.

Eddington a donc réussi à supprimer la difficulté qui

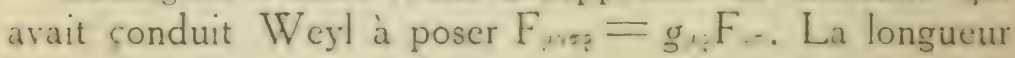


nulle peut ne pas rester nulle par déplacement parallèle; peu importe, puisque le cône lumineux est défini par la seule équation invariante absolue qu'on puisse former.

La courbure constante. - Prenant les scalaires des deux membres de (16-21), on a, en tout point

$$
B=R=4 .
$$

qui, dans le vide, devient

$$
\mathrm{R}=4 \% \text {. }
$$

Il est évident que \% n'est pas nul, car il n'y aurait plus de systeme de jauges naturel. Nous sommes donc directcment conduits à la conception de la courbure constante ct de l'espace fermé. La constance de la courbure est imposée par la condition qui détermine le système de jauges : cela revient à dire que le système naturel consiste à prendre pour jauge en chaque point le rayon de courbure d'Univers; ou encore que tout objet est une portion déterminée et constante de l'Univers; que tout électron doit avoir pour rayon une fraction constante du rayon de courbure d'Univers au point ou il se trouve. Si le rayon d'Univers changeait d'un point à l'autre - par rapport à un sur-étalon que nous ne saurions d'ailleurs imaginer - l'électron, nos instruments, nous-mêmes, tout changerait dans le même rapport ; par conséquent le rayon de courbure doit nous apparaitre comme constant.

"R a la même valeur partout. Si l'on conserve le point de vue de la théorie d'Einstein, en séparant le champ de gravitation et le champ électromagnétique, et si l'on appelle courbure le scalaire $\mathrm{R}$ qui ne fait pas intervenir les $\mathrm{S}_{\%}^{*}$, on doit considèrer les électrons comme des déformations locales. L'électron devient une région de forte courbure, bien que, 
avec le système naturel, " $\mathrm{R}$ ait la même valeur que dans


de $\mathrm{R}$. doivent étre considérables dans l'électron; autrement dit, le champ électrique doit y ètre colossal.

Matiere cl électricité. - Pour identifier la substance contenue dans l'espace, nous devons chercher les tenseurs géométriques qui correspondent aux tenseurs physiques. Ces tenseurs n'ont d'ailleurs pas besoin d'être absolus car nous utilisons le systéme de jauges naturel (aux faibles erreurs près dues à l'ambigüî́ résultant de la non-intégrabilité des longueurs) et nous n'arons aucune raison de penser que les lois de notre science se conserveraient toutes dans un systeme de jauges arbitraire.

Tout d'abord, rien n'est changé à la loi de la gravitation dans la matière, car la généralisation de W Weyl-Eddington n̈introduit pas de nouveau tenseur à divergence nulle auquel on puisse identifier $T_{\cdots}^{\prime}$

Le tenseur F .. des forces électrique et magnétique doit satisfaire le premier groupe des équatıons de Maxwell généralisées, et ces équations deviennent des identités (14-6) si F... est le rotationnel d'un recteur. Nous voyons quill n'y a qu'un seul tenseur géométrique que nous puissions identifier avec le tenseur des forces électrique et magnétique, c'est celui que nous arons précisément désigné par $F$. (16-17). Le vecteur $\div$ dont $F_{2}$. est le rotationnel, est le potentiel.

Le recteur courant-densité de charge doit satisfaire à la loi expérimentale de conservation de l'électricité. II faut donc que $J^{\prime}=0$; cette cquation devient une identic si $\mathrm{J}$ ' est la divergence d'un tenseur symétrique gaurhe irntrevariant; nous devons donc identitier J' avec la divergence 
de $\mathrm{F}^{\prime}$; nous obtenons ainsi le second groupe de Maxwell. L'électron. - Nous arons vu (note 14, n³) qu'il est impossible de corsstruire un électron et par conséquent de la maticre à partir du champ électromagnétique seul; on sait d'ailleurs que l'électron ne peut exister qu'en admettant des forces de cohésion non-maxwelliennes (pressions de Poincarél. Si l'on almet la continuité dans la structure géométrique de l'Univers, il est possible de calculer en chaque point le scalaire $T$ du tenseur total d'énergie c'est-d-dire "la densité de substance". L'expression est d'ailleurs assez compliquée. Le résultat intéressant est le suivant: il est permis de penser que les forces de cohésion, jusqu'alors mystérieuses, qui permettent l'existence de l'électron sont les $S_{\text {,. }}^{-}$qui ajoutées aux composantes $1: 1$ du champ de gravitation constituent les forces absolues I ( 1 (éq. 16-13). L'union du tenseur de gravitation g., et du tenseur d'électricité $\mathrm{S}^{-}$(ou plutôt d'un tenseur :... à partir duquel sont formés les $S_{j}^{\prime} I$ ou plus simplement, si l'on admet la restriction de Weyl, l'union de $g$.. et de $\tau$, suffit pour rendre compte de l'existence des électrons et de la matière, alors que le champ de gravitation et les forces maxwelliennes $F$, ne suffisaient pas.

" Le potentiel électromagnétique a en lui quelque chose de fondamental qui disparait quand on en prend le rotationnel pour obtenir la force électromagnétique observable (Eddington).

Toutefois dans le problème de la matière, il ne parait pas exact de supposer une structure d'Univers continue, car l'expérience nous a révélé l'étrange loi des quanta. Les 
lois du continu ne sont probablement pas applicables à l'électron, mais on ne voit pas oì intervient une discontinuité dans la constitution de l'électron.

Les généralisations de $W$ eyl et d'Edding on complètent la théorie d'Einstein sans l'altérer. On peut, dans la description géométrique de l'Univers, considérer séparément le tenseur $B_{\ldots}$ ( ou $g_{g}$ ) qui décrit le champ de gravitation et le tenseur $F_{\text {. }}$ qui décrit le champ électromagnétique : c'est ce quavait fait Einstein; l' " intervalle d'Einstein est absolu, puisque c'est l'invariant absolu B.. $d x_{n} d x$. L'œurre d'Einstein reste donc intacte; elle n'est atteinte en rien par l'ambiguïté que l'existence du champ électromagnétique apportc dans la comparaison des longueurs. 


\section{BIBILIOGRAPHIE}

Lorentz - Einstein - Minkowski. - Das Relativitutsprinzip; nouvelle édition (Teubner).

Ce livre contient les plus importants mémoires originaux.

A. Einstern. - La théorie de la relutivité restreinte et généralisée, mise à la portée de tout le monde. Traduit par $\mathrm{M}^{1 ! !} \mathrm{J}$. Rouvière.

$L$ 'éther de la relativité, traduit par M. Solovine.

P. Langevin. - L'évolution de l'espace et du temps (Scientia, 1911).

Le temps, l'espace et la causalité dans la physique moderne. Bulletin de la société de philosophie (19 oct. 1911).

L'inerlie de l'énergie. Conférence à la société de physique (17 mars 1913) publiée dans le Journal de physique.

Le principe de relativité. Bulletin de la société des électriciens, déc. 1919.

H. Weyl. - Raum, Zeit, Materie, édition 1921 (Springer, éd.).

Max von Laue. - Die Relativitätstheoric, t. I (1919) et 1. II (1922) (Vieweg \& Sohn, éd.).

A.-S. Eddincton. - Report on the reluticity thenry of gratitation (1920) (publié par la Société de physique de Londres).

Espace, temps et gravitation (1921) traduit par M. Jacques Ro:signol.

De SitTer. - On Einstein theory of Gravitation, and its astronomical consequences. Monthly notices, oct. 1916, déc. 1916, now. 1917.

Jean Becquerel. - Le principe de relaticité et la théorie de lu garavitation (1922) (Gauthier Villars, éd.). 

PAYOT \& C', 100, Boulcrart Saint-Germain, PARIS-VI .

EMILE MEYERSON

\section{DE L'EXPLICATION}
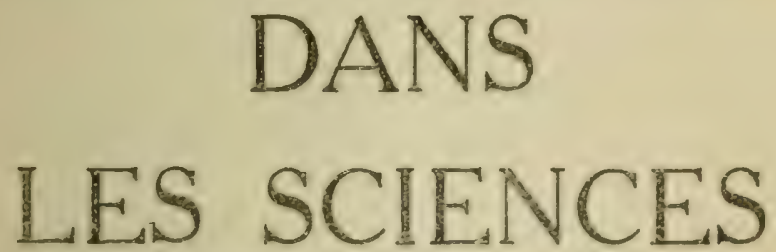

2 vol. in-S ensemble.. . . . . . . 40 fr.

Fspligur les pliénomènes, expliquer l"univers, est le but suprême de la sirnce, comme de la philnopphie elle-méme. Mais il y a untinomie entre lin-trument de nolre connaisance, la raison, qui ne peut [mocéder que par le moyen de lidentité, et la réalité du monde, qui exappe perpetuellement à lidentification et dont l'esence e.t d'ene irrationaelle. La téalité nou est-e!le done insaisissable? Tet est le problène capial çue poece et quélucide avec profondeur M. Emile


desra deomais sevvir, pour employer une expression de lauteur dan

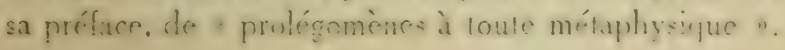


PAYOT \& C

\section{ACHALME}

Directeur de Laboratoire à l'École des Hautes-Éludes.

\section{LES ÉDIFICES \\ PHYSICO-CHIMIQUES}

TOME I
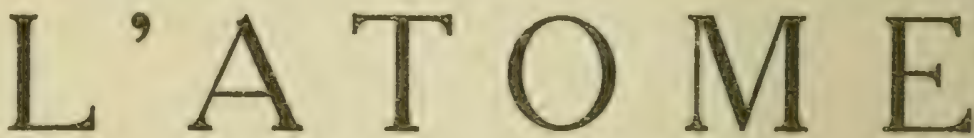

SA STRUCTURE, SA FORME

Un volume in- 8 avec gravures de M. Raoul LECLERC. . . . . . . . . $15 \mathrm{fr}$.

... Un livre excellent, clair et hardi. On y trouvera les derniers progrès que nous devons à Perrin, à Weis, à J.J. Thomson. On y trouvera davantage: un essai de concrétisation. L'ensemble de ce volume constitue un tout séduisant, rationnel, propre a faciliter la spéculation scientifique ou philosophiq̨ue et bien adapté à nos connaissances.

(L'Aclion Française.) 


\section{$L A$ « COLLECTION PAYOT \# S'EST ASSURÉE DE}

\section{LA COLLABORATION DE MM.}

HENRI ANDOYER, Membre de l'Institut, Professeur à la Sorbonne.

PAUL APPELL, Membre de l'Institut, Recteur de l'Université de Paris.

$L^{*}-C^{1}$ E. ARIĖS, Correspondant de l'Institut.

AUGUSTE AUDOLLENT, Doyen de la Faculté des Lettres de Clermont.

ERNEST BABELON, Membre de l'Institut, Professeur au Collège de France.

JEAN BABELON, Aitaché au Cabinet des Médailles.

E. BAILLAUD, Membre de l'Institut, Directeur de l'Observatoire de Paris.

LOUIS BARTHOU, de l'Académie Française, ancien Président du Conseil, Ministre de la Guerre.

JEAN BECQUEREL, Professeur au Muséum National d'Hisioire Naturelle.

PAUL BECQUEREL, Docteur ès Sciences chargé d'Enseignement pratique à la Sorbonne.

HENRY BÉRENGER, Sénateur.

A. BERTHOUD, Próesseur à I'Université de Neuchîtel.

GABRIEL BERTRAND, Professeur à la Sorbonne et à l'Institut Pasteur.

MAURICE BESNIER, Professeur à l'Université de Caen.

G. BIGOURDAN, Membre de l'Institut, Astronome de l'Observatoire de Paris.

F. BOQUET, Astronome de l'Observatoire de Paris.

Abbé J. BOSON, Docteur en Philologie orientale.

D PIERRE BOULAN.

PIERRE BOUTROUX, Professeur au Co'lège de France.

EDMOND BOUTY, Membre de l'Institut, Professeur à la Sorbonne.

E. BRANLY, Membre de l'Institut, Professeur à l'Institut Catholique. .

ÉMILE BREHIER, Maitre de conférences à la Sorbonne.

BRETIGNIERE, Professeur à l'École nationale d'Agriculture de Grignon.

M. BRILLOUIN, Professeur au Collège de France.

BUSSARD, Professeur à l'École nationale d'Horticulture de Versailles.

RENÉ CANAT, Docteur ès Lettres, Professeur de Rhétorique supérieure au Lycée Louis-le-Grand.

Dr CAPITAN, Membre de l'Académie de Médec ne, Professeur' au Collège de France, Professeur à l'École d'Anthropologie.

J. CARCOPINO, Ancien Membre de l'École de Rome, Professeur à la Sorbonne.

A. CARTAULT, Professeur à la Sorbonne.

EUGENE CAVAIGNAC, Professeur à l'Université de Strastourz.

G. CHAUVEAUD, Directeur de laboratoire à l'École des Hautes-Études.

HENRI CHERMEZON, Docteur ès Sciences, Chef de travaux à la Faculté des Sciences de Strasbourg.

C'DE CIVRIEUX.

$D^{r}$ G. CONTENAU, chargé de Missions archéologiques en Syrie.

HENRI CORDIER, Membre de l'Institut, Prof' à l'École des Langues orientales. M. COURANT, Professeur à I'Université de Lyon.

MAURICE CROISET, Membre de I'Institut, Professeur au Collège de France. ÉDOUARD CUQ, Membre de l'Institut, Professeur à la Faculté de Droit.

L. DAUPHINÉ, Docteur ès Sciences, chargé d'Enseignement pratique à la Sorbonne.

P. DECHAMBRE, Membre de l'Académie d'Agriculture.

MAURICE DELACRE, Membre de l'Académie Royale de Belgique, Professeur à I'Université de Gand. 
M. DELAFOSSE, ancien Gouverneur des Colonies, Professeur à l'École coloniale. Comte DELAMARRE DE MONCHAUX, Président de la Section d'Áviculture de la Société des Agriculteurs de France.

CH. DEPÉRET, Membre de l'Institut, Doyen de la Faculté des Sciences de Lyon. G. DESDEVISES DU DÉZERT, Professeur à l'Univ. de Clermont-Ferrand. CH. DIEHL, Membre de l'Institut, Professeur à la Sorbonne.

G.DOTTIN, Correspondant del'Institut, Doyen de la Faculté des Lettres de Rennes. ALBERT DUFOURCQ, Professeur à l'Université de Bordeaux.

CH. DUGAS, Professeur à l'Université de Montpellier.

JEAN DUHAMEL, Secrétaire du Comité Central des Houillères de France. Comte P. DURRIEU, Membre de l'Institut, Conservateur honoraire au Louvre* RENÉ DUSSAUD, Conservateur au Louvre, Professeur à l'École du Louvre. JEAN DYBOWSKI, Membre de l'Académie d'Agriculture.

CAMILLE ENLART, Directeur du Musée de Sculpture Comparée.

$C^{\ddagger}$ ÉMILE ESPÉRANDIEU, Membre de l'Institut.

P. FABIA, Correspondant de l'Institut, Professeur à l'Université de Lyon.

HENRI FOCILLON, Professeur à la Faculté des Lettres de l'Université de Lyon. G. FOUGËES, ancien Directeur de l'École d'Athènes, Professeur à la Sorbonne. A. GASTOUÉ, Professeur à la Schola-Cantorum. E.-F. GAUTIER, Professeur à la Faculté des Lettres d'Alger. PROSPER GERVAIS, Président de l'Académie d'Agriculture. ÉTIENNE GILSON, Professeur à l'Université de Strasbourg. CHARLES GIRARD, Membre de l'Académie d'Agriculture. PAUL GIRARD, Membre de l'Institut, Professeur à la Sorbonne GUSTAVE GLOTZ, Membre de l'Institut, Professeur à la Sorbonne PAUL GOUY, Directeur de "La Viticulture exportatrice".

A. GRENIER, Professeur à l'Université de Strasbourg.

PIERRE GRILLET, Agrégé de l'Université.

GEORGES GROMAIRE, Professeur au Lycée Buffon.

A. GUILLAND, Professeur à l'École Polytechnique de Zurich.

J HATZFELD, Professeur à I'Université de Bordeaux.

L HAUTECCEUR, Professeur à l'Université de Caen.

HENRI HAUVETTE, Professeur à la Sorbonne.

FÉLIX HENNEGUY, Membre de l'Ins'itut, Membre de l'Académie de Médecine. HENRI HITIER, Membre de l'Académie d'Agriculture.

JOSEPH HITIER, Professeur à la Faculté de Droit de Paris et à I'Institut national agronomique.

PIERRE JOUGUET, Correspondant de l'Institut, Professeur à la Sorbonne.

KAYSER, $D^{r}$ des Laboratoires de Fermentation à l'lnstitut national agronomique. G. LACOUR-GAYET, Membre de l'Institut, Professeur à l'École Polytechnique. A. LACROIX, Secrétaire Perpétuel de l'Académie des Sciences.

HENRY LAFOSSE, Membre de l'Académie d'Agriculture.

L. DE LAUNAY, Membre de l'Institut, Pro'esseur à l'École des Mines.

G. LE CARDONNEL.

HENRI LECHAT, Correspondant de l'Institut, Professeur à I'Université de Lyon.

E. LECLAINCHE, Membre de l'Institut, Inspecteur général au Ministère de TAgriculture.

G. LE GENTIL, Professeur à la Sorbonne. 


\section{COLLABORATEURS DE LA « COLLECTION PAYOT}

LOUIS LEGER, Membre de l'Institut, Professeur au Collège de France.

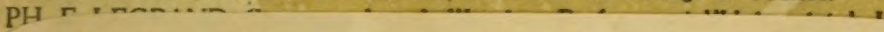

Becqueral, Jean

Expose elementaire de 1 a theorie d'Einstein et de sa generalisation. 
